\title{
SYMMETRIC MONOIDAL STRUCTURE ON NON-COMMUTATIVE MOTIVES
}

\author{
by
}

Denis-Charles Cisinski \& Gonçalo Tabuada

\begin{abstract}
In this article we further the study of non-commutative motives, initiated in $[11,42]$. Our main result is the construction of a symmetric monoidal structure on the localizing motivator Mot $\mathrm{dg}_{\mathrm{dg}}^{\mathrm{loc}}$ of $\mathrm{dg}$ categories. As an application, we obtain: (1) a computation of the spectra of morphisms in Mot ${ }_{\mathrm{dg}}^{\text {loc }}$ in terms of nonconnective algebraic $K$-theory; (2) a fully-faithful embedding of Kontsevich's category $\mathrm{KMM}_{k}$ of non-commutative mixed motives into the base category $\operatorname{Mot}_{\mathrm{dg}}^{\text {loc }}(e)$ of the localizing motivator; (3) a simple construction of the Chern character maps from non-connective algebraic $K$-theory to negative and periodic cyclic homology; (4) a precise connection between Toën's secondary $K$-theory and the Grothendieck ring of $\mathrm{KMM}_{k} ;(5)$ a description of the Euler characteristic in $\mathrm{KMM}_{k}$ in terms of Hochschild homology.
\end{abstract}

\section{Contents}

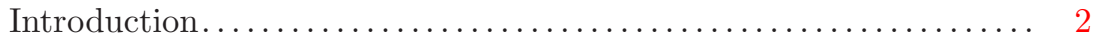

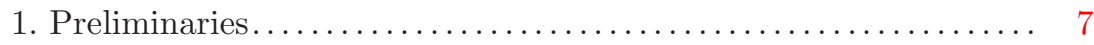

2. Background on $d g$ categories....................... 8

3. Homotopically finitely presented $\mathrm{dg}$ categories.............. 14

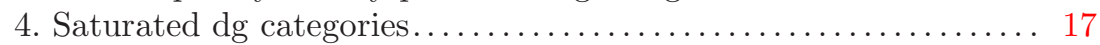

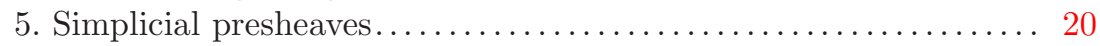

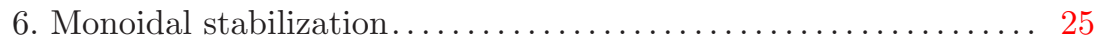

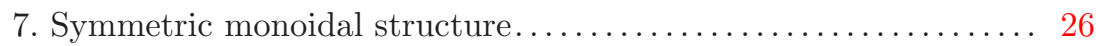

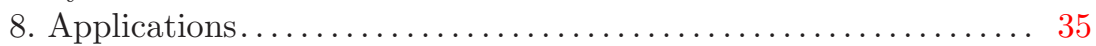

A. Grothendieck derivators......................... 45

2000 Mathematics Subject Classification. - 19D35, 19D55, 18D10, 18D20, 19E08.

Key words and phrases. - Non-commutative motives, Non-commutative algebraic geometry, Non-connective algebraic $K$-theory, Secondary $K$-theory, Hochschild homology, Negative cyclic homology, Periodic cyclic homology.

The first named author was partially supported by the ANR (grant No. ANR-07-BLAN-042). The second named author was partially supported by the Estímulo à Investigação Award 2008 - Calouste Gulbenkian Foundation. 
References........................................ 54

\section{Introduction}

Dg categories. - A differential graded $(=d g)$ category, over a commutative base ring $k$, is a category enriched over complexes of $k$-modules (morphisms sets are such complexes) in such a way that composition fulfills the Leibniz rule: $d(f \circ g)=(d f) \circ$ $g+(-1)^{\operatorname{deg}(f)} f \circ(d g)$. Dg categories enhance and solve many of the technical problems inherent to triangulated categories; see Keller's ICM adress [30]. In non-commutative algebraic geometry in the sense of Bondal, Drinfeld, Kapranov, Kontsevich, Toën, Van den Bergh, . . [3, 4, 16, 17, 33, 34, 35, 48], they are considered as dg-enhancements of derived categories of (quasi-)coherent sheaves on a hypothetic non-commutative space.

Localizing invariants. - All the classical (functorial) invariants, such as Hochschild homology, cyclic homology and its variants (periodic, negative, ...), algebraic $K$-theory, and even topological Hochschild homology and topological cyclic homology (see [45]), extend naturally from $k$-algebras to dg categories. In order to study all these classical invariants simultaneously, the second named author introduced in [42] the notion of localizing invariant. This notion, that we now recall, makes use of the language of Grothendieck derivators [28], a formalism which allows us to state and prove precise universal properties; consult Appendix A. Let $L: \mathrm{HO}$ (dgcat) $\rightarrow \mathbb{D}$ be a morphism of derivators, from the derivator associated to the Morita model structure of dg categories (see §2.3), to a triangulated derivator (in practice, $\mathbb{D}$ will be the derivator associated to a stable model category $\mathcal{M}$, and $L$ will come from a functor dgcat $\rightarrow \mathcal{M}$ which sends derived Morita equivalences to weak equivalences in $\mathcal{M}$ ). We say that $L$ is a localizing invariant (see Definition 7.3 ) if it preserves filtered homotopy colimits as well as the terminal object, and sends Drinfeld exact sequences of dg categories

$$
\mathcal{A} \longrightarrow \mathcal{B} \longrightarrow \mathcal{C} \mapsto L(\mathcal{A}) \longrightarrow L(\mathcal{B}) \longrightarrow L(\mathcal{C}) \longrightarrow L(\mathcal{A})[1]
$$

to distinguished triangles in the base category $\mathbb{D}(e)$ of $\mathbb{D}$. Thanks to the work of Keller [31, 32], Schlichting [41], Thomason-Trobaugh [47], and BlumbergMandell [2], all the mentioned invariants satisfy localization ${ }^{(1)}$, and so give rise to localizing invariants. In [42], the second named author proved that there exists a localizing invariant

$$
\mathcal{U}_{\mathrm{dg}}^{\text {loc }}: \mathrm{HO}(\text { dgcat }) \longrightarrow \text { Mot }_{\mathrm{dg}}^{\text {loc }}
$$

with values in a strong triangulated derivator (see $\S$ A.3), such that given any strong triangulated derivator $\mathbb{D}$, we have an induced equivalence of categories

$$
\left(\mathcal{U}_{\mathrm{dg}}^{\text {loc }}\right)^{*}: \underline{\operatorname{Hom}}_{!}\left(\operatorname{Mot}_{\mathrm{dg}}^{\mathrm{loc}}, \mathbb{D}\right) \stackrel{\sim}{\longrightarrow} \underline{\operatorname{Hom}}_{\mathrm{loc}}(\mathrm{HO}(\text { dgcat }), \mathbb{D}) \text {. }
$$

\footnotetext{
${ }^{(1)}$ In the case of algebraic $K$-theory we consider its non-connective version.
} 
The left-hand side denotes the category of homotopy colimit preserving morphisms of derivators, and the right-hand side the category of localizing invariants.

Because of this universality property, which is a reminiscence of motives, $\mathcal{U}_{\mathrm{dg}}^{\text {loc }}$ is called the universal localizing invariant, $\operatorname{Mot}_{\mathrm{dg}}^{\text {loc }}$ the localizing motivator, and the base category $\operatorname{Mot}_{\mathrm{dg}}^{\mathrm{loc}}(e)$ of the localizing motivator the category of non-commutative motives over $k$.

Symmetric monoidal structure. - The purpose of this article is to develop a new ingredient in the theory of non-commutative motives: symmetric monoidal structures. The tensor product extends naturally from $k$-algebras to $\mathrm{dg}$ categories, giving rise to a symmetric monoidal structure on $\mathrm{HO}$ (dgcat); see Theorem 2.23. Therefore, it is natural to consider localizing invariants which are symmetric monoidal. Examples include Hochschild homology and the mixed and periodic complex constructions; see Examples 7.9-7.11. The main result of this article is the following.

Theorem 0.1. - (see Theorem 7.5) The localizing motivator Mot ${ }_{\mathrm{dg}}^{\mathrm{loc}}$ carries a canonical symmetric monoidal structure $-\otimes^{\mathbb{L}}$-, making the universal localizing invariant $\mathcal{U}_{\mathrm{dg}}^{\text {loc }}$ symmetric monoidal. Moreover, this tensor product preserves homotopy colimits in each variable and is characterized by the following universal property: given any strong triangulated derivator $\mathbb{D}$, endowed with a monoidal structure which preserves homotopy colimits, we have an induced equivalence of categories

$$
\left(\mathcal{U}_{\mathrm{dg}}^{\text {loc }}\right)^{*}: \underline{\operatorname{Hom}}_{!}^{\otimes}\left(\operatorname{Mot}_{\mathrm{dg}}^{\mathrm{loc}}, \mathbb{D}\right) \stackrel{\sim}{\longrightarrow} \underline{\operatorname{Hom}}_{\mathrm{loc}}^{\otimes}(\mathrm{HO}(\text { dgcat }), \mathbb{D}),
$$

where the left-hand side stands for the category of symmetric monoidal homotopy colimit preserving morphisms of derivators, while the right-hand side stands for the category symmetric monoidal morphisms of derivators which are also localizing invariants; see $\S A .5$. Furthermore, Mot $\mathrm{d}_{\mathrm{dg}}^{\mathrm{loc}}$ admits an explicit symmetric monoidal Quillen model.

The proof of Theorem 0.1 is based on an alternative description of Mot ${ }_{\mathrm{dg}}^{\text {loc }}$, with two complementary aspects: a constructive one, and another given by universal properties.

The constructive aspect, i.e. the construction of an explicit symmetric monoidal Quillen model for Mot $\mathrm{t}_{\mathrm{dg}}^{\mathrm{loc}}$, is described in the main body of the text. The key starting point is the fact that homotopically finitely presented dg categories are stable under derived tensor product; see Theorem 3.4. This allows us to obtain a small symmetric monoidal category which "generates" the entire Morita homotopy category of dg categories. Starting from this small monoidal category, we then construct a specific symmetric monoidal Quillen model for each one of the derivators used in the construction of Mot ${ }_{\mathrm{dg}}^{\mathrm{loc}}$; see $\S 7.1$.

The characterization of Mot ${ }_{\mathrm{dg}}^{\text {loc }}$ by its universal property (as stated in Theorem 0.1), relies on general results and constructions in the theory of Grothendieck derivators, and is described in the appendix. We develop some general results concerning the behavior of monoidal structures under classical operations: Kan extension (see Theorem A.3), left Bousfield localization (see Proposition A.9) and stabilization (see 
Theorem A.15). Using these general results, we then characterize by a precise universal property each one of the Quillen models used in the construction of the new symmetric monoidal Quillen model for Mot ${ }_{\mathrm{dg}}^{\mathrm{loc}}$; see $\S 7.1$.

Let us now describe some applications of Theorem 0.1 .

Non-connective $K$-theory. - As mentioned above, non-connective algebraic $K$ theory $\mathbb{K}(-)$ is an example of a localizing invariant. In [11] the authors proved that this invariant becomes co-representable in $\operatorname{Mot}_{\mathrm{dg}}^{\text {loc }}(e)$ by the unit object $\mathcal{U}_{\mathrm{dg}}^{\text {loc }}(\underline{k})$ (where $\underline{k}$ corresponds to $k$, seen as a dg category with one object). In other words, given any $\operatorname{dg}$ category $\mathcal{A}$, we have a natural isomorphism in the stable homotopy category of spectra :

$$
\mathbb{R} \operatorname{Hom}\left(\mathcal{U}_{\mathrm{dg}}^{\mathrm{loc}}(\underline{k}), \mathcal{U}_{\mathrm{dg}}^{\mathrm{loc}}(\mathcal{A})\right) \simeq \mathbb{K}(\mathcal{A}) .
$$

A fundamental problem of the theory of non-commutative motives is the computation of the (spectra of) morphisms in the category of non-commutative motives between any two objects. Using the monoidal structure of Theorem 0.1 we extend the above natural isomorphism (0.0.1), and thus obtain a partial solution to this problem.

Theorem 0.2. - (see Theorem 8.2) Let $\mathcal{B}$ be a saturated dg category in the sense of Kontsevich, i.e. its complexes of morphisms are perfect and $\mathcal{B}$ is perfect as a bimodule over itself; see Definition 4.1. Then, for every small $d g$ category $\mathcal{A}$, we have a natural isomorphism in the stable homotopy category of spectra

$$
\mathbb{R} \operatorname{Hom}\left(\mathcal{U}_{\mathrm{dg}}^{\mathrm{loc}}(\mathcal{B}), \mathcal{U}_{\mathrm{dg}}^{\mathrm{loc}}(\mathcal{A})\right) \simeq \mathbb{K}(\operatorname{rep}(\mathcal{B}, \mathcal{A})),
$$

where rep $(-,-)$ denotes the internal Hom-functor in the Morita homotopy category of $d g$ categories; see $§ 2.4$.

Given a quasi-compact and separated $k$-scheme $X$, there is a natural dg category $\operatorname{perf}(X)$ which enhances the category of perfect complexes (i.e. of compact objects) in the (unbounded) derived category $\mathcal{D}_{q c o h}(X)$ of quasi-coherent sheaves on $X$; see Example 4.5. Moreover, when $X$ is smooth and proper, the $\operatorname{dg}$ category $\operatorname{perf}(X)$ is a saturated dg category. In this geometrical situation, we have the following computation.

Proposition 0.3. - (see Proposition 8.3) Given smooth and proper $k$-schemes X and $Y$, we have a natural isomorphism in the stable homotopy category of spectra

$$
\mathbb{R H o m}\left(\mathcal{U}_{\mathrm{dg}}^{\text {loc }}(\operatorname{perf}(X)), \mathcal{U}_{\mathrm{dg}}^{\text {loc }}(\operatorname{perf}(Y))\right) \simeq \mathbb{K}(X \times Y),
$$

where $\mathbb{I}(X \times Y)$ denotes the non-connective algebraic $K$-theory spectrum of $X \times Y$.

Kontsevich's category of non-commutative mixed motives. - In his noncommutative algebraic geometry program [33, 34, 35], Kontsevich introduced the category $\mathrm{KMM}_{k}$ of non-commutative mixed motives; see $\S 8.2$. Roughly, $\mathrm{KMM}_{k}$ is obtained by taking a formal Karoubian triangulated envelope of the category of saturated dg categories (with algebraic $K$-theory of bimodules as morphism sets). Using Theorem 0.2 , we prove the following result. 
Proposition 0.4. - (see Proposition 8.5) There is a natural fully-faithful embedding (enriched over spectra) of Kontsevich's category $\mathrm{KMM}_{k}$ of non-commutative mixed motives into the base category $\operatorname{Mot}_{\mathrm{dg}}^{\mathrm{loc}}(e)$ of the localizing motivator. The essential image is the thick triangulated subcategory spanned by motives of saturated dg categories.

Note that, in contrast with Kontsevich's ad hoc definition, the category $\operatorname{Mot}_{\mathrm{dg}}^{\mathrm{loc}}(e)$ of non-commutative motives is defined purely in terms of precise universal properties.

Chern characters. - Let

$$
E: \mathrm{HO}(\text { dgcat }) \longrightarrow \mathbb{D}
$$

be a symmetric monoidal localizing invariant. Thanks to Theorem 0.1 there is a (unique) symmetric monoidal homotopy colimit preserving morphism of derivators $E_{\text {gm }}$ which makes the diagram

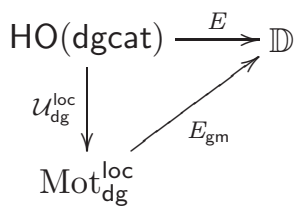

commute (up to unique 2-isomorphism). We call $E_{\mathrm{gm}}$ the geometric realization of $E$. If $E(\underline{k}) \simeq \mathbf{1}$ denotes the unit of $\mathbb{D}$, we can also associate to $E$ its absolute realization

$$
E_{\mathrm{abs}}:=\mathbb{R H o m}_{\mathbb{D}}\left(\mathbf{1}, E_{\mathrm{gm}}(-)\right) \quad(\text { see Definition 8.6) }
$$

Proposition 0.5. - (see Proposition 8.7) The geometric realization of E induces a canonical Chern character

$$
\mathbb{I K}(-) \Rightarrow \mathbb{R} \operatorname{Hom}(\mathbf{1}, E(-)) \simeq E_{\mathrm{abs}}\left(\mathcal{U}_{\mathrm{dg}}^{\mathrm{loc}}(-)\right) .
$$

Here, $\mathbb{K}(-)$ and $E_{\mathrm{abs}}\left(\mathcal{U}_{\mathrm{dg}}^{\mathrm{loc}}(-)\right)$ are two morphisms of derivators defined on $\mathrm{HO}$ (dgcat).

Let $\mathcal{A}$ be a small dg category. When $E$ is given by the mixed complex construction (see Example 7.10), the absolute realization of $\mathcal{U}_{\mathrm{dg}}^{\mathrm{loc}}(\mathcal{A})$ identifies with the negative cyclic homology complex $H C^{-}(\mathcal{A})$ of $\mathcal{A}$. Therefore by Proposition 0.5 , we obtain a canonical Chern character

$$
\mathbb{K}(-) \Rightarrow H C^{-}(-)
$$

from non-connective $K$-theory to negative cyclic homology; see Example 8.10. When $E$ is given by the composition of the mixed complex construction with the periodization procedure (see Example 7.11), the absolute realization of $\mathcal{U}_{\mathrm{dg}}^{\mathrm{loc}}(\mathcal{A})$ identifies with the periodic cyclic homology complex $H P(\mathcal{A})$ of $\mathcal{A}$. Therefore by Proposition 0.5 , we obtain a canonical Chern character

$$
\mathbb{I K}(-) \Rightarrow H P(-)
$$

from non-connective $K$-theory to periodic cyclic homology; see Example 8.11. 
Toën's secondary $K$-theory. - In his sheaf categorification program [50, 52], Toën introduced a "categorified" version of algebraic $K$-theory, named secondary $K$ theory. Given a commutative ring $k$, the secondary $K$-theory ring $K_{0}^{(2)}(k)$ of $k$ is roughly the quotient of the free abelian group on derived Morita isomorphism classes of saturated dg categories, by the relations $[\mathcal{B}]=[\mathcal{A}]+[\mathcal{C}]$ coming from Drinfeld exact sequences $\mathcal{A} \rightarrow \mathcal{B} \rightarrow \mathcal{C}$. The multiplication is induced from the derived tensor product; see §8.4. As Toën pointed out in [50], one of the motivations for the study of this secondary $K$-theory is its expected connection with an hypothetical Grothendieck ring of motives in the non-commutative setting. Thanks to the monoidal structure of Theorem 0.1 we are now able to make this connection precise.

Definition 0.6. - (see Definition 8.16) Given a commutative ring $k$, the Grothendieck ring $\mathcal{K}_{0}(k)$ of non-commutative motives over $k$ is the Grothendieck ring of Kontsevich mixed motives (i.e. of the thick triangulated subcategory of $\operatorname{Mot}_{\mathrm{dg}}^{\text {loc }}(e)$ generated by the objects $\mathcal{U}_{\mathrm{dg}}^{\text {loc }}(\mathcal{A})$, where $\mathcal{A}$ runs over the family of saturated dg categories).

Kontsevich's saturated dg categories can be characterized conceptually as the dualizable objects in the Morita homotopy category; see Theorem 4.8. Therefore, since the universal localizing invariant $\mathcal{U}_{\mathrm{dg}}^{\text {loc }}$ is symmetric monoidal we obtain a ring homomorphism

$$
\Phi(k): K_{0}^{(2)}(k) \longrightarrow \mathcal{K}_{0}(k) .
$$

Moreover, the Grothendieck ring of Definition 0.6 is non-trivial (see Remark 8.17), functorial in $k$ (see Remark 8.18), and the ring homomorphism $\Phi(k)$ is functorial in $k$ and surjective "up to cofinality" (see Remark 8.19). Furthermore, any realization of $K_{0}^{(2)}(k)$ (i.e. ring homomorphism $K_{0}^{(2)}(k) \rightarrow R$ ), which is induced from a symmetric monoidal localizing invariant, factors through $\Phi(k)$. An interesting example is provided by Toën's rank map

$$
r k_{0}: K_{0}^{(2)}(k) \longrightarrow K_{0}(k) \quad \text { (see Remark 8.19). }
$$

Euler characteristic. - Recall that, in any symmetric monoidal category, we have the notion of Euler characteristic $\chi(X)$ of a dualizable object $X$ (see Definition 8.20). In the symmetric monoidal category of non-commutative motives we have the following computation.

Proposition 0.7. - (see Proposition 8.24) Let $\mathcal{A}$ be a saturated dg category. Then, $\chi\left(\mathcal{U}_{\mathrm{dg}}^{\mathrm{loc}}(\mathcal{A})\right)$ is the element of the Grothendieck group $K_{0}(k)$ which is associated to the (perfect) Hochschild homology complex $H H(\mathcal{A})$ of $\mathcal{A}$.

When $k$ is the field of complex numbers, the Grothendieck ring $K_{0}(\mathbb{C})$ is naturally isomorphic to $\mathbb{Z}$ and the Hochschild homology of a smooth and proper $k$-scheme $X$ agrees with the Hodge cohomology $H^{*}\left(X, \Omega_{X}^{*}\right)$ of $X$. Therefore, when we work over $\mathbb{C}$, and if perf $(X)$ denotes the (saturated) dg category of perfect complexes over $X$, the Euler characteristic of $\mathcal{U}_{\mathrm{dg}}^{\text {loc }}(\operatorname{perf}(X))$ is the classical Euler characteristic of $X$. 
Acknowledgments : The authors are very grateful to Bertrand Toën for sharing his insights on saturated dg categories and to Maxim Kontsevich for comments on a previous version which motivated the creation of subsection 8.3. The second named author would like also to thank Paul Balmer and Christian Haesemeyer for some conversations. This article was initiated at the Institut Henri Poincaré in Paris. The authors would like to thank this institution for its hospitality and stimulating working environment.

\section{Preliminaries}

1.1. Notations. - Throughout the article we will work over a fixed commutative and unital base ring $k$.

We will denote by $\mathcal{C}(k)$ be the category of (unbounded) complexes of $k$-modules; see $[24, \S 2.3]$. We will use co-homological notation, i.e. the differential increases the degree. The category $\mathcal{C}(k)$ is a symmetric monoidal model category (see [24, Definition 4.2.6]), where one uses the projective model structure for which weak equivalences are quasi-isomorphisms and fibrations are surjections; see [24, Proposition 4.2.13].

The category of sets will be denoted by Set, the category of simplicial sets by sSet, and the category of pointed simplicial sets by sSet•; see [20, $\S \mathrm{I}]$. The categories sSet and sSet. are symmetric monoidal model categories; see [20, Proposition 4.2.8]. The weak equivalences are the maps whose geometric realization is a weak equivalence of topological spaces, the fibrations are the Kan-fibrations, and the cofibrations are the inclusion maps.

We will denote by $S p^{\mathbb{N}}$ the category of spectra and by $\mathrm{Sp}^{\Sigma}$ the category of symmetric spectra (of pointed simplicial sets); see [27].

Finally, the adjunctions will be displayed vertically with the left (resp. right) adjoint on the left- (resp. right-) hand side.

1.2. Triangulated categories. - Throughout the article we will use the language of triangulated categories. The reader unfamiliar with this language is invited to consult Neeman's book [39] or Verdier's original monograph [53]. Recall from [39, Definition 4.2.7] that given a triangulated category $\mathcal{T}$ admitting arbitrary small coproducts, an object $G$ in $\mathcal{T}$ is called compact if for each family $\left\{Y_{i}\right\}_{i \in I}$ of objects in $\mathcal{T}$, the canonical morphism

$$
\bigoplus_{i \in I} \operatorname{Hom}_{\mathcal{T}}\left(G, Y_{i}\right) \stackrel{\sim}{\longrightarrow} \operatorname{Hom}_{\mathcal{T}}\left(G, \bigoplus_{i \in I} Y_{i}\right)
$$

is invertible. We will denote by $\mathcal{T}_{c}$ the category of compact objects in $\mathcal{T}$.

1.3. Quillen model categories. - Throughout the article we will use freely the language of Quillen model categories. The reader unfamiliar with this language is invited to consult Goerss \& Jardine [20], Hirschhorn [23], Hovey [24], or Quillen's 
original monograph [40]. Given a model category $\mathcal{M}$, we will denote by $\mathrm{Ho}(\mathcal{M})$ its homotopy category and by

$$
\operatorname{Map}(-,-): \mathrm{Ho}(\mathcal{M})^{\mathrm{op}} \times \mathrm{Ho}(\mathcal{M}) \longrightarrow \mathrm{Ho}(\mathrm{sSet})
$$

its homotopy function complex; see [23, Definition 17.4.1].

1.4. Grothendieck derivators. - Throughout the article we will use the language of Grothendieck derivators. Derivators allow us to state and prove precise universal properties and to dispense with many of the technical problems one faces in using Quillen model categories. Since this language may be less familiar to the reader, we revise it in the appendix. Given a model category $\mathcal{M}$, we will denote by $\mathrm{HO}(\mathcal{M})$ its associated derivator; see $\S A .2$. Any triangulated derivator $\mathbb{D}$ is canonically enriched over spectra; see $\S A .9$. We will denote by $\mathbb{R} \operatorname{Hom}_{\mathbb{D}}(X, Y)$ the spectrum of maps from $X$ to $Y$ in $\mathbb{D}$. When there is no ambiguity, we will write $\mathbb{R} \operatorname{Hom}(X, Y)$ instead of $\mathbb{R} \operatorname{Hom}_{\mathbb{D}}(X, Y)$.

\section{Background on dg categories}

In this section we collect and recall the notions and results on the (homotopy) theory of dg categories which will be used throughout the article; see [43, 44, 48]. At the end of the section we prove a new result: the derivator associated to the Morita model structure carries a symmetric monoidal structure; see Theorem 2.23. This result is the starting point of the article, since it allow us to define symmetric monoidal localizing invariants; see Definition 7.6.

Definition 2.1. - A small dg category $\mathcal{A}$ is a $\mathcal{C}(k)$-category; see [5, Definition 6.2.1]. Recall that this consists of the following data:

- a set of objects $\operatorname{obj}(\mathcal{A})$ (usually denoted by $\mathcal{A}$ itself);

- for each ordered pair of objects $(x, y)$ in $\mathcal{A}$, a complex of $k$-modules $\mathcal{A}(x, y)$;

- for each ordered triple of objects $(x, y, z)$ in $\mathcal{A}$, a composition morphism in $\mathcal{C}(k)$

$$
\mathcal{A}(y, z) \otimes \mathcal{A}(x, y) \longrightarrow \mathcal{A}(x, z),
$$

satisfying the usual associativity condition;

- for each object $x$ in $\mathcal{A}$, a morphism $k \rightarrow \mathcal{A}(x, x)$, satisfying the usual unit condition with respect to the above composition.

Definition 2.2. - A $d g$ functor $F: \mathcal{A} \rightarrow \mathcal{B}$ is a $\mathcal{C}(k)$-functor; see [5, Definition 6.2.3]. Recall that this consists of the following data:

- a map of sets $F: \operatorname{obj}(\mathcal{A}) \longrightarrow \operatorname{obj}(\mathcal{B})$;

- for each ordered pair of objects $(x, y)$ in $\mathcal{A}$, a morphism in $\mathcal{C}(k)$

$$
F(x, y): \mathcal{A}(x, y) \longrightarrow \mathcal{B}(F x, F y),
$$

satisfying the usual unit and associativity conditions. 
Notation 2.3. - We denote by dgcat the category of small dg categories.

\subsection{Dg cells. -}

(i) Let $\underline{k}$ be the dg category with one object $*$ and such that $\underline{k}(*, *):=k$ (in degree zero). Note that given a small dg category $\mathcal{B}$, there is a bijection between the set of $\mathrm{dg}$ functors from $\underline{k}$ to $\mathcal{B}$ and the set of objects of $\mathcal{B}$.

(ii) For $n \in \mathbb{Z}$, let $S^{n}$ be the complex $k[n]$ (with $k$ concentrated in degree $n$ ) and $D^{n}$ the mapping cone on the identity of $S^{n-1}$. We denote by $\mathcal{S}(n)$ the dg category with two objects 1 and 2 such that $\mathcal{S}(n)(1,1)=k, \mathcal{S}(n)(2,2)=k, \mathcal{S}(n)(2,1)=$ $0, \mathcal{S}(n)(1,2)=S^{n}$ and composition given by multiplication. We denote by $\mathcal{D}(n)$ the dg category with two objects 3 and 4 such that $\mathcal{D}(n)(3,3)=k, \mathcal{D}(n)(4,4)=$ $k, \mathcal{D}(n)(4,3)=0, \mathcal{D}(n)(3,4)=D^{n}$ and composition given by multiplication.

(iii) For $n \in \mathbb{Z}$, let $\iota(n): \mathcal{S}(n-1) \rightarrow \mathcal{D}(n)$ be the dg functor that sends 1 to 3,2 to 4 and $S^{n-1}$ to $D^{n}$ by the identity on $k$ in degree $n-1$ :

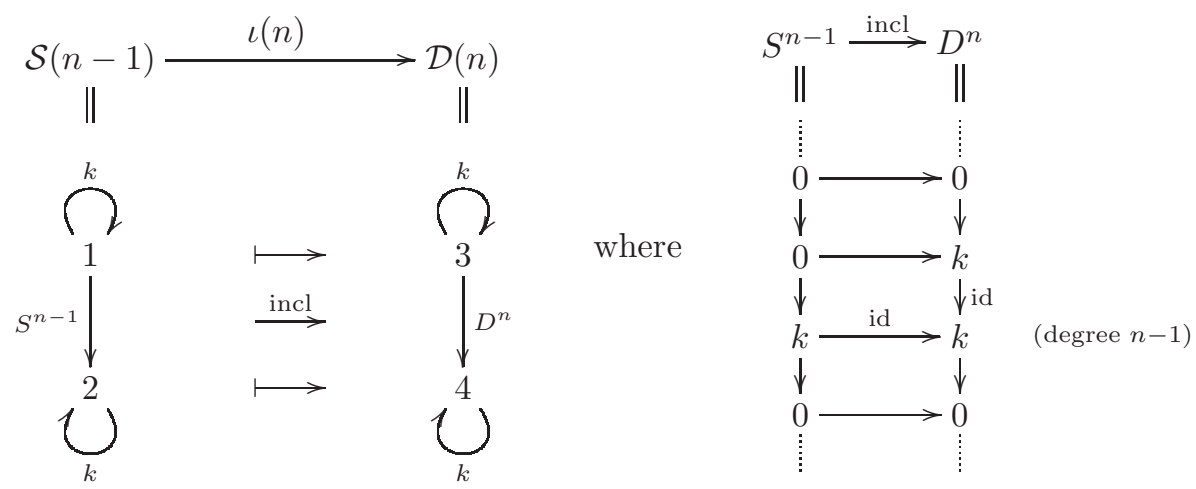

Notation 2.4. - Let $I$ be the set consisting of the dg functors $\{\iota(n)\}_{n \in \mathbb{Z}}$ and the $\operatorname{dg}$ functor $\emptyset \rightarrow \underline{k}$ (where the empty dg category $\emptyset$ is the initial object in dgcat).

Definition 2.5. - A dg category $\mathcal{A}$ is called a $d g$ cell (resp. a finite $d g$ cell) if the unique dg functor $\emptyset \rightarrow \mathcal{A}$ can be expressed as a transfinite (resp. finite) composition of pushouts of dg functors in $I$; see [23, Definition 10.5.8(2)].

2.2. Dg modules. - Let $\mathcal{A}$ be a small (fixed) dg category.

Definition 2.6. - - The category $\mathrm{H}^{0}(\mathcal{A})$ has the same objects as $\mathcal{A}$ and morphisms given by $\mathrm{H}^{0}(\mathcal{A})(x, y):=\mathrm{H}^{0}(\mathcal{A}(x, y))$, where $\mathrm{H}^{0}(-)$ denotes the 0 -th co-homology group functor.

- The opposite dg category $\mathcal{A}^{\text {op }}$ of $\mathcal{A}$ has the same objects as $\mathcal{A}$ and complexes of morphisms given by $\mathcal{A}^{\mathrm{op}}(x, y):=\mathcal{A}(y, x)$.

- A right $d g \mathcal{A}$-module $M$ (or simply a $\mathcal{A}$-module) is a $\operatorname{dg}$ functor $M: \mathcal{A}^{\mathrm{op}} \rightarrow \mathcal{C}_{\mathrm{dg}}(k)$ with values in the $\mathrm{dg}$ category $\mathcal{C}_{\mathrm{dg}}(k)$ of complexes of $k$-modules. 
We denote by $\mathcal{C}(\mathcal{A})$ the category of right $\operatorname{dg} \mathcal{A}$-modules. Its objects are the right $\operatorname{dg} \mathcal{A}$-modules and its morphisms are the natural transformations of dg functors; see $[30, \S 2.3]$. The differential graded structure of $\mathcal{C}_{\mathrm{dg}}(k)$ makes $\mathcal{C}(\mathcal{A})$ naturally into a $\mathrm{dg}$ category; see $[30, \S 3.1]$ for details. We denote by $\mathcal{C}_{\mathrm{dg}}(\mathcal{A})$ this $\mathrm{dg}$ category of right $\mathrm{dg} \mathcal{A}$-modules. Recall from [30, Theorem 3.2] that $\mathcal{C}(\mathcal{A})$ carries a standard projective model structure. A morphism $M \rightarrow M^{\prime}$ in $\mathcal{C}(\mathcal{A})$ is a weak equivalence, resp. a fibration, if for any object $x$ in $\mathcal{A}$, the induced morphism $M(x) \rightarrow M^{\prime}(x)$ is a weak equivalence, resp. a fibration, in the projective model structure on $\mathcal{C}(k)$. In particular, every object is fibrant. Moreover, the dg category $\mathcal{C}_{\mathrm{dg}}(\mathcal{A})$ endowed with this model structure is a $\mathcal{C}(k)$-model category in the sense of [24, Definition 4.2.18].

Notation 2.7. - - - We denote by $\mathcal{D}_{\mathrm{dg}}(\mathcal{A})$ the full $\mathrm{dg}$ subcategory of fibrant and cofibrant objects in $\mathcal{C}_{\mathrm{dg}}(\mathcal{A})$.

- We denote by $\mathcal{D}(\mathcal{A})$ the derived category of $\mathcal{A}$, i.e. the localization of $\mathcal{C}(\mathcal{A})$ with respect to the class of weak equivalences. Note that $\mathcal{D}(\mathcal{A})$ is a triangulated category with arbitrary small coproducts. Recall from $\S 1.2$ that we denote by $\mathcal{D}_{c}(\mathcal{A})$ the category of compact objects in $\mathcal{D}(\mathcal{A})$.

Since $\mathcal{C}_{\mathrm{dg}}(\mathcal{A})$ is a $\mathcal{C}(k)$-model category, [48, Proposition 3.5] implies that we have a natural equivalence of (triangulated) categories

$$
\mathrm{H}^{0}\left(\mathcal{D}_{\mathrm{dg}}(\mathcal{A})\right) \simeq \mathcal{D}(\mathcal{A})
$$

We denote by

$$
\underline{h}: \mathcal{A} \longrightarrow \mathcal{C}_{\mathrm{dg}}(\mathcal{A}) \quad x \mapsto \mathcal{A}(-, x),
$$

the classical Yoneda $d g$ functor; see $[5, \S 6.3]$. Since the $\mathcal{A}$-modules $\mathcal{A}(-, x), x$ in $\mathcal{A}$, are fibrant and cofibrant in the projective model structure, the Yoneda functor factors through the inclusion $\mathcal{D}_{\mathrm{dg}}(\mathcal{A}) \subset \mathcal{C}_{\mathrm{dg}}(\mathcal{A})$. Thanks to the above equivalence $(2.2 .1)$, we obtain an induced fully-faithful functor

$$
\underline{h}: \mathrm{H}^{0}(\mathcal{A}) \hookrightarrow \mathcal{D}(\mathcal{A}) \quad x \mapsto \mathcal{A}(-, x) .
$$

Finally, let $F: \mathcal{A} \rightarrow \mathcal{B}$ be a dg functor. As shown in $[48, \S 3]$ it induces a restriction/extension of scalars Quillen adjunction (on the left-hand side)

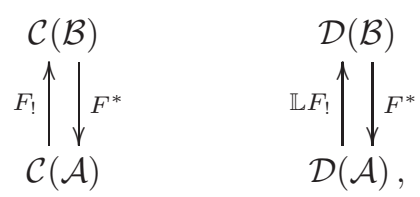

which can be naturally derived (on the right-hand side). 


\subsection{Morita model structure. -}

Definition 2.8. - A dg functor $F: \mathcal{A} \rightarrow \mathcal{B}$ is a called a derived Morita equivalence if the restriction of scalars functor $F^{*}: \mathcal{D}(\mathcal{B}) \stackrel{\sim}{\rightarrow} \mathcal{D}(\mathcal{A})$ is an equivalence of triangulated categories. Equivalently, $F$ is a derived Morita equivalence if the extension of scalars functor $\mathbb{L} F_{!}: \mathcal{D}(\mathcal{A}) \stackrel{\sim}{\rightarrow} \mathcal{D}(\mathcal{B})$ is an equivalence of triangulated categories.

Theorem 2.9. - The category dgcat carries a cofibrantly generated Quillen model structure (called the Morita model structure), whose weak equivalences are the derived Morita equivalences. Moreover, its set of generating cofibrations is the set I of Notation 2.4.

Proof. — See [43, Théorème 5.3].

Notation 2.10. - We denote by Hmo the homotopy category hence obtained.

Recall from [43, Remarque 5.4] that the fibrant objects of the Morita model structure (called the Morita fibrant $d g$ categories) are the $\operatorname{dg}$ categories $\mathcal{A}$ for which the image of the induced functor

$$
\underline{h}: \mathrm{H}^{0}(\mathcal{A}) \hookrightarrow \mathcal{D}(\mathcal{A}) \quad x \mapsto \mathcal{A}(-, x)
$$

is stable under (co-)suspensions, cones and direct factors.

\subsection{Monoidal structure. -}

Definition 2.11. - Let $\mathcal{A}_{1}$ and $\mathcal{A}_{2}$ be small dg categories. The tensor product $\mathcal{A}_{1} \otimes \mathcal{A}_{2}$ of $\mathcal{A}_{1}$ with $\mathcal{A}_{2}$ is defined as follows: the set of objects of $\mathcal{A}_{1} \otimes \mathcal{A}_{2}$ is obj $\left(\mathcal{A}_{1}\right) \times$ $\operatorname{obj}\left(\mathcal{A}_{2}\right)$ and given objects $\left(x, x^{\prime}\right)$ and $\left(y, y^{\prime}\right)$ in $\mathcal{A}_{1} \otimes \mathcal{A}_{2}$, we set

$$
\left(\mathcal{A}_{1} \otimes \mathcal{A}_{2}\right)\left(\left(x, x^{\prime}\right),\left(y, y^{\prime}\right)\right)=\mathcal{A}_{1}(x, y) \otimes \mathcal{A}_{2}\left(x^{\prime}, y^{\prime}\right) .
$$

Remark 2.12. - The tensor product of dg categories gives rise to a symmetric monoidal structure on dgcat, with unit object the dg category $\underline{k}$ (see $\S 2.1$ ). Moreover, this model structure is easily seen to be closed. However, the model structure of Theorem 2.9 endowed with this symmetric monoidal structure is not a symmetric monoidal model category, as the tensor product of two cofibrant objects in dgcat is not cofibrant in general; see $[48, \S 4]$. Nevertheless, the tensor product can be derived into a bifunctor

$$
-\otimes^{\mathbb{L}}-: \mathrm{Hmo} \times \mathrm{Hmo} \longrightarrow \mathrm{Hmo} \quad\left(\mathcal{A}_{1}, \mathcal{A}_{2}\right) \mapsto Q\left(\mathcal{A}_{1}\right) \otimes \mathcal{A}_{2}=: \mathcal{A}_{1} \otimes^{\mathbb{L}} \mathcal{A}_{2},
$$

where $Q\left(\mathcal{A}_{1}\right)$ is a Morita cofibrant resolution of $\mathcal{A}_{1}$.

Now, let $\mathcal{B}$ and $\mathcal{A}$ be small $\mathrm{dg}$ categories. For any object $x$ in $\mathcal{B}$, we have a $\mathrm{dg}$ functor $\mathcal{A} \rightarrow \mathcal{B}^{\mathrm{op}} \otimes \mathcal{A}$. It sends an object $y$ in $\mathcal{A}$ to $(x, y)$ and for each ordered pair of objects $(y, z)$ in $\mathcal{A}$, the morphism in $\mathcal{C}(k)$

$$
\mathcal{A}(y, z) \longrightarrow\left(\mathcal{B}^{\mathrm{op}} \otimes \mathcal{A}\right)((x, y),(x, z))=\mathcal{B}^{\mathrm{op}}(x, x) \otimes \mathcal{A}(y, z)
$$


is given by the tensor product of the unit morphism $k \rightarrow \mathcal{B}^{\mathrm{op}}(x, x)$ with the identity morphism on $\mathcal{A}(y, z)$. Take a Morita cofibrant resolution $Q\left(\mathcal{B}^{\text {op }}\right)$ of $\mathcal{B}^{\text {op }}$ with the same set of objects; see [48, Proposition 2.3(2)]. Since $\mathcal{B}^{\text {op }}$ and $Q\left(\mathcal{B}^{\text {op }}\right)$ have the same set of objects, one sees that for any object $x$ in $\mathcal{B}$, we have a dg functor

$$
i_{x}: \mathcal{A} \longrightarrow Q\left(\mathcal{B}^{\mathrm{op}}\right) \otimes \mathcal{A}=: \mathcal{B}^{\mathrm{op}} \otimes^{\mathbb{L}} \mathcal{A} .
$$

Definition 2.13. - Let $\mathcal{A}$ be a small dg category. A $\mathcal{A}$-module $M$ is called perfect if it is compact as an object in the derived category $\mathcal{D}(\mathcal{A})$. We denote by $\mathcal{C}_{\mathrm{dg}}(\mathcal{A})^{\text {pe }}$ the dg category of perfect $\mathcal{A}$-modules, and put

$$
\operatorname{perf}(\mathcal{A})=\mathcal{C}_{\mathrm{dg}}(\mathcal{A})^{\mathrm{pe}} \cap \mathcal{D}_{\mathrm{dg}}(\mathcal{A})
$$

We thus have an equivalence of triangulated categories

$$
\mathrm{H}^{0}(\operatorname{perf}(\mathcal{A})) \simeq \mathcal{D}_{c}(\mathcal{A})
$$

Let $\mathcal{B}$ and $\mathcal{A}$ be small dg categories. A $\left(\mathcal{B}^{\text {op }} \otimes \mathbb{L} \mathcal{A}\right)$-module $X$ is said to be locally perfect over $\mathcal{B}$ if, for any object $x$ in $\mathcal{B}$, the $\mathcal{A}$-module $i_{x}^{*}(X)$ (see (2.4.1) and $\left.\S 2.2\right)$ is perfect. We denote by $\mathcal{C}_{\mathrm{dg}}\left(\mathcal{B}^{\mathrm{op}} \otimes^{\mathbb{L}} \mathcal{A}\right)^{\mathrm{lpe}}$ the dg category of locally perfect $\left(\mathcal{B}^{\mathrm{op}} \otimes^{\mathbb{L}} \mathcal{A}\right)$-modules over $\mathcal{B}$, and put

$$
\operatorname{rep}(\mathcal{B}, \mathcal{A})=\mathcal{C}_{\mathrm{dg}}\left(\mathcal{B}^{\text {op }} \otimes^{\mathbb{L}} \mathcal{A}\right)^{\operatorname{lpe}} \cap \mathcal{D}_{\mathrm{dg}}\left(\mathcal{B}^{\text {op }} \otimes^{\mathbb{L}} \mathcal{A}\right) .
$$

Note that $\mathrm{H}^{0}(\operatorname{rep}(\mathcal{B}, \mathcal{A}))$ is canonically equivalent to the full triangulated subcategory of $\mathcal{D}\left(\mathcal{B}^{\text {op }} \otimes^{\mathbb{L}} \mathcal{A}\right)$ spanned by the locally perfect $\left(\mathcal{B}^{\text {op }} \otimes^{\mathbb{L}} \mathcal{A}\right)$-modules over $\mathcal{B}$.

Remark 2.14. - For any dg category $\mathcal{A}$, the Yoneda embedding $\underline{h}: \mathcal{A} \rightarrow \operatorname{perf}(\mathcal{A})$ is a derived Morita equivalence, and $\operatorname{perf}(\mathcal{A})$ is Morita fibrant. This construction thus provides us a canonical fibrant replacement functor for the Morita model structure.

Theorem 2.15 (Toën). - Given small dg categories $\mathcal{B}$ and $\mathcal{A}$, we have a natural bijection

$$
\operatorname{Hom}_{\mathrm{Hmo}}(\mathcal{B}, \mathcal{A}) \simeq \operatorname{Iso} \mathrm{H}^{0}(\operatorname{rep}(\mathcal{B}, \mathcal{A}))
$$

(where Iso $\mathcal{C}$ stands for the set of isomorphism classes of objects in $\mathcal{C}$ ). Moreover, given small dg categories $\mathcal{A}_{1}, \mathcal{A}_{2}$, and $\mathcal{A}_{3}$, the composition in $\mathrm{Hmo}$ corresponds to the derived tensor product of bimodules:

$$
\begin{array}{ccc}
\text { Iso } \mathrm{H}^{0}\left(\operatorname{rep}\left(\mathcal{A}_{1}, \mathcal{A}_{2}\right)\right) \times \operatorname{Iso} \mathrm{H}^{0}\left(\operatorname{rep}\left(\mathcal{A}_{2}, \mathcal{A}_{3}\right)\right) & \longrightarrow & \operatorname{Iso} \mathrm{H}^{0}\left(\operatorname{rep}\left(\mathcal{A}_{1}, \mathcal{A}_{3}\right)\right) \\
([X],[Y]) & \longmapsto & {\left[X \otimes_{\mathcal{A}_{2}}^{\mathbb{L}} Y\right] .}
\end{array}
$$

Proof. - See [48, Corollary 4.8] and [43, Remarque 5.11].

Theorem 2.16 (Toën). - The derived tensor product $-\otimes^{\mathbb{L}}-$ on Hmo admits the bifunctor

$$
\mathrm{rep}(-,-): \mathrm{Hmo}^{\text {op }} \times \mathrm{Hmo} \longrightarrow \mathrm{Hmo}
$$

as an internal Hom-functor.

Proof. - See [48, Theorem 6.1] and [43, Remarque 5.11]. 
Corollary 2.17. - Given small dg categories $\mathcal{A}_{1}, \mathcal{A}_{2}$ and $\mathcal{A}_{3}$, we have a natural isomorphism in $\mathrm{Ho}(\mathrm{sSet})$

$$
\operatorname{Map}\left(\mathcal{A}_{1} \otimes^{\mathbb{L}} \mathcal{A}_{2}, \mathcal{A}_{3}\right) \simeq \operatorname{Map}\left(\mathcal{A}_{1}, \operatorname{rep}\left(\mathcal{A}_{2}, \mathcal{A}_{3}\right)\right) .
$$

Proof. - See [48, Corollary 6.4] and [43, Remarque 5.11].

Remark 2.18. - Observe that, when $\mathcal{B}=\underline{k}$, the dg category $\mathcal{B}^{\text {op }} \otimes^{\mathbb{L}} \mathcal{A}$ identifies with $\mathcal{A}$, which gives a natural isomorphism in $\mathrm{Hmo}$ :

$$
\operatorname{rep}(\underline{k}, \mathcal{A}) \simeq \operatorname{perf}(\mathcal{A}) .
$$

We finish this section by showing how to endow the derivator associated to the Morita model structure on dg categories with a symmetric monoidal structure; see Theorem 2.23. Recall from $\S 2.4$ that the Morita model structure is not a monoidal model structure and so we cannot apply the general Proposition A.2. We sidestep this difficulty by introducing the notion of $k$-flat $d g$ category.

Definition 2.19. - A complex of $k$-modules is called $k$-flat if for any acyclic complex $N$, the complex $M \otimes N$ is acyclic.

A dg category is called $k$-flat if for each ordered pair of objects $(x, y)$ in $\mathcal{A}$, the complex $\mathcal{A}(x, y)$ is $k$-flat.

Notation 2.20. - We denote by dgcat flat $_{\text {the }}$ category of $k$-flat dg categories.

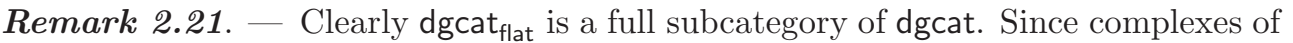
$k$-flat modules are stable under tensor product, the category dgcat flat $_{\text {is }}$ a symmetric monoidal full subcategory of dgcat (see §2.4). Moreover, the tensor product

$$
-\otimes-: \text { dgcat }_{\text {flat }} \times \text { dgcat }_{\text {flat }} \longrightarrow \text { dgcat }_{\text {flat }}
$$

preserves derived Morita equivalences (in both variables).

Notation 2.22. - We denote by $\mathrm{HO}$ (dgcat) the derivator associated to the Morita model structure on dg categories; see Theorem 2.9.

Theorem 2.23. - The derivator $\mathrm{HO}$ (dgcat) carries a symmetric monoidal structure $-\otimes^{\mathbb{L}}-;$ see $\S A .5$. Moreover, the induced symmetric monoidal structure on $\mathrm{HO}(\mathrm{dgcat})(e)=\mathrm{Hmo}$ coincides with the one described in Remark 2.12.

Proof. - Let $\mathrm{HO}\left(\mathrm{dgcat}_{\text {flat }}\right)=\operatorname{dgcat}_{\text {flat }}\left[\mathcal{M o r}^{-1}\right]$ be the prederivator associated to the class $\mathcal{M}$ or of derived Morita equivalences in dgcat $_{\text {flat }}$; see $\S A .1$. Since dgcat flat is a symmetric monoidal category, and the tensor product preserves the class $\mathcal{M}$ or, i.e. $\mathcal{M o r} \otimes$ Mor $\subset$ Mor, the prederivator $\mathrm{HO}\left(\right.$ dgcat $\left._{\text {flat }}\right)$ carries a natural symmetric monoidal structure; see $\S$ A.5. Now, choose a functorial Morita cofibrant resolution functor

$$
Q: \text { dgcat } \longrightarrow \text { dgcat } \quad Q \Rightarrow \text { Id . }
$$


Thanks to [48, Proposition 2.3(3)] every cofibrant dg category is $k$-flat, and so we obtain functors

$$
i: \text { dgcat }_{\text {flat }} \hookrightarrow \text { dgcat } \quad Q: \text { dgcat } \longrightarrow \text { dgcat }_{\text {flat }}
$$

and natural transformations

$$
i \circ Q \Rightarrow \operatorname{Id} \quad Q \circ i \Rightarrow \operatorname{Id} .
$$

Since the functors $i$ and $Q$ preserve derived Morita equivalences and the above natural transformations (2.4.4) are objectwise derived Morita equivalences, we obtain by 2functoriality (see $\S$ A.1) morphisms of derivators

$$
i: \mathrm{HO}\left(\text { dgcat }_{\text {flat }}\right) \longrightarrow \mathrm{HO}(\text { dgcat }) \quad Q: \mathrm{HO}(\text { dgcat }) \longrightarrow \mathrm{HO}\left(\text { dgcat }_{\text {flat }}\right)
$$

which are quasi-inverse to each other. Using this equivalence of derivators, we transport the monoidal structure from $\mathrm{HO}\left(\right.$ dgcat $\left._{\text {flat }}\right)$ to $\mathrm{HO}$ (dgcat). The fact that the induced monoidal structure on $\mathrm{HO}($ dgcat $)(e)=\mathrm{Hmo}$ coincides with the one described in Remark 2.12 is now clear.

Proposition 2.24. - The tensor product on $\mathrm{HO}$ (dgcat) of Theorem 2.23 preserves homotopy colimits in each variable.

Proof. — This follows immediately from Corollary 2.17.

\section{Homotopically finitely presented dg categories}

In this section we give a new characterization of homotopically finitely presented dg categories; see Theorem 3.3(3). Using this new characterization we show that homotopically finitely presented dg categories are stable under derived tensor product; see Theorem 3.4. This stability result will play a key role in the construction of a symmetric monoidal model structure on the category of non-commutative motives; see Section 7.

Proposition 3.1. - For any small $d g$ category $\mathcal{A}$ and $n \in \mathbb{Z}$, we have natural isomorphisms in $\mathrm{Hmo}$

$$
\mathcal{S}(n)^{\mathrm{op}} \otimes \mathcal{A} \simeq \mathcal{S}(n)^{\mathrm{op}} \otimes^{\mathbb{L}} \mathcal{A} \simeq \operatorname{rep}(\mathcal{S}(n), \mathcal{A})
$$

Proof. - Since $\mathcal{S}(n) \simeq \mathcal{S}(n)^{\text {op }}$ is cofibrant, we obtain the following isomorphism in $\mathrm{Hmo}$

$$
\mathcal{S}(n)^{\mathrm{op}} \otimes^{\mathbb{L}} \mathcal{A} \simeq \mathcal{S}(n)^{\mathrm{op}} \otimes \mathcal{A}
$$

By construction, the $\mathrm{dg}$ category $\mathcal{S}(n)$ is also locally perfect, i.e. its complexes of morphisms are perfect complexes of $k$-modules; see [51, Definition 2.4(1)]. We obtain then by [51, Lemma 2.8] the following inclusion

$$
\mathcal{S}(n)^{\mathrm{op}} \otimes \mathcal{A} \simeq \operatorname{perf}\left(\mathcal{S}(n)^{\mathrm{op}} \otimes^{\mathbb{L}} \mathcal{A}\right) \subset \operatorname{rep}(\mathcal{S}(n), \mathcal{A}) .
$$


Let us now prove the converse inclusion. Observe that we have two natural dg functors

$$
\begin{aligned}
& i_{1}: \mathcal{A} \longrightarrow \mathcal{S}(n)^{\mathrm{op}} \otimes \mathcal{A} \quad i_{2}: \mathcal{A} \longrightarrow \mathcal{S}(n)^{\mathrm{op}} \otimes \mathcal{A} \\
& x \longmapsto \quad(1, x) \quad x \longmapsto \quad(2, x)
\end{aligned}
$$

and the category of $\left(\mathcal{S}(n)^{\text {op }} \otimes \mathcal{A}\right)$-modules identifies with the category of morphisms of degree $n$ in $\mathcal{C}(\mathcal{A})$, i.e. to the category of triples $\left(M, M^{\prime}, f\right)$, where $M$ and $M^{\prime}$ are $\mathcal{A}$ modules, and $f: M \longrightarrow M^{\prime}[n]$ is a morphism of $\mathcal{A}$-modules. Under this identification, we obtain the following extension of scalars functors (see $\S 2.2$ ):

$$
\begin{aligned}
\left(i_{1}\right) !: \mathcal{C}(\mathcal{A}) \longrightarrow \mathcal{C}\left(\mathcal{S}(n)^{\mathrm{op}} \otimes \mathcal{A}\right) & M \longmapsto\left(M, M[-n], 1_{M}\right) \\
\left(i_{2}\right) !: \mathcal{C}(\mathcal{A}) \longrightarrow \mathcal{C}\left(\mathcal{S}(n)^{\mathrm{op}} \otimes \mathcal{A}\right) & M \longmapsto(0, M, 0) .
\end{aligned}
$$

Now, let $X$ be an object in $\operatorname{rep}(\mathcal{S}(n), \mathcal{A})$. Note that $X$ corresponds to a cofibration

$$
f: M \succ M^{\prime}[n]
$$

in $\mathcal{C}(\mathcal{A})$ between cofibrant and perfect $\mathcal{A}$-modules (see Definition 2.13). Consider the following short exact sequence of morphisms of $\mathcal{A}$-modules

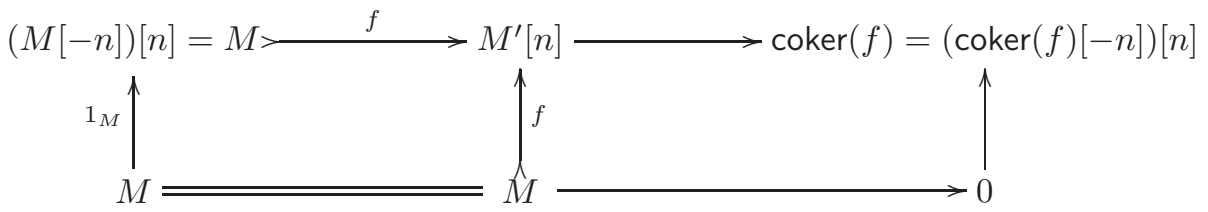

where coker $(f)$ denotes the cokernel of $f$ in the category $\mathcal{C}(\mathcal{A})$. Since $M$ and $M^{\prime}[n]$ are cofibrant and perfect $\mathcal{A}$-modules, and $f$ is a cofibration, the $\mathcal{A}$-module coker $(f)$ is also cofibrant and perfect. Perfect modules are stable under suspension, and so coker $(f)[-n]$ is a perfect (and cofibrant) $\mathcal{A}$-module. We have natural isomorphisms :

$$
\left(i_{1}\right) !(M) \simeq\left(M, M[-n], 1_{M}\right) \quad \text { and } \quad\left(i_{2}\right) !(\operatorname{coker}(f)[-n]) \simeq(0, \operatorname{coker}(f)[-n], 0) .
$$

Since the extension of scalars functors $\mathbb{L}\left(i_{1}\right)$ ! and $\mathbb{L}\left(i_{2}\right)$ ! preserve perfect modules, these two objects are perfect. Finally, since $\operatorname{perf}\left(\mathcal{S}(n)^{\mathrm{op}} \otimes \mathcal{A}\right)$ is stable under extensions in $\mathcal{D}_{\mathrm{dg}}\left(\mathcal{S}(n)^{\text {op }} \otimes \mathcal{A}\right)$, and in the above short exact sequence (3.0.5) the left and right vertical morphisms belong to $\operatorname{perf}\left(\mathcal{S}(n)^{\mathrm{op}} \otimes \mathcal{A}\right)$, we conclude that the object $X$ also belongs to $\operatorname{perf}\left(\mathcal{S}(n)^{\text {op }} \otimes \mathcal{A}\right)$.

Definition 3.2 ([51, Definition 2.1(3)]). - Let $\mathcal{M}$ be a Quillen model category. An object $X$ in $\mathcal{M}$ is called homotopically finitely presented if for any filtered system of objects $\left\{Y_{j}\right\}_{j \in J}$ in $\mathcal{M}$, the induced map

$$
\underset{j \in J}{\operatorname{hocolim}} \operatorname{Map}\left(X, Y_{j}\right) \longrightarrow \operatorname{Map}\left(X, \underset{j \in J}{\operatorname{hocolim}} Y_{j}\right)
$$

is an isomorphism in $\mathrm{Ho}(\mathrm{sSet})$.

Theorem 3.3. - Let $\mathcal{B}$ be a small dg category. In the Morita model structure (see Theorem 2.9) the following conditions are equivalent: 
(1) The $d g$ category $\mathcal{B}$ is homotopically finitely presented;

(2) The $d g$ category $\mathcal{B}$ is a retract in $\mathrm{Hmo}$ of a finite $d g$ cell (see Definition 2.5);

(3) For any filtered system $\left\{\mathcal{A}_{j}\right\}_{j \in J}$ of $d g$ categories, the induced morphism

$$
\underset{j \in J}{\operatorname{hocolim}} \operatorname{rep}\left(\mathcal{B}, \mathcal{A}_{j}\right) \stackrel{\sim}{\longrightarrow} \operatorname{rep}\left(\mathcal{B}, \underset{j \in J}{\operatorname{hocolim}} \mathcal{A}_{j}\right)
$$

is an isomorphism in $\mathrm{Hmo}$.

Proof. - (1) $\Leftrightarrow(2)$ : This equivalence follows from [42, Proposition 5.2] and [42, Example 5.1].

$(3) \Rightarrow(1)$ : Let $\left\{\mathcal{A}_{j}\right\}_{j \in J}$ be a filtered system of dg categories. By hypothesis, we have an induced isomorphism in $\mathrm{Hmo}$

$$
\underset{j \in J}{\operatorname{hocolim}} \operatorname{rep}\left(\mathcal{B}, \mathcal{A}_{j}\right) \stackrel{\sim}{\longrightarrow} \operatorname{rep}\left(\mathcal{B}, \underset{j \in J}{\operatorname{hocolim}} \mathcal{A}_{j}\right) .
$$

Thanks to Corollary 2.17 we have, for any $\operatorname{dg}$ category $\mathcal{A}$, natural isomorphisms in $\mathrm{Ho}(\mathrm{sSet})$ :

$$
\operatorname{Map}(\mathcal{B}, \mathcal{A}) \simeq \operatorname{Map}\left(\underline{k} \otimes^{\mathbb{L}} \mathcal{B}, \mathcal{A}\right) \simeq \operatorname{Map}(\underline{k}, \operatorname{rep}(\mathcal{B}, \mathcal{A})) .
$$

The dg category $\underline{k}$ is a finite dg cell, and so using equivalence $(1) \Leftrightarrow(2)$ we conclude that $\underline{k}$ is homotopically finitely presented. Therefore, by applying the functor

$$
\operatorname{Map}(\underline{k},-): \mathrm{Hmo} \longrightarrow \mathrm{Ho}(\mathrm{sSet})
$$

to the above isomorphism (3.0.7), we obtain an induced isomorphism in $\mathrm{Ho}(\mathrm{sSet})$

$$
\underset{j \in J}{\operatorname{hocolim}} \operatorname{Map}\left(\mathcal{B}, \mathcal{A}_{j}\right) \stackrel{\sim}{\longrightarrow} \operatorname{Map}\left(\mathcal{B}, \underset{j \in J}{\operatorname{hocolim}} \mathcal{A}_{j}\right) .
$$

This shows that $\mathcal{B}$ is homotopically finitely presented.

$(2) \Rightarrow(3)$ : The class of objects in Hmo which satisfy condition (3) is clearly stable under retracts. Moreover, given a small $\operatorname{dg}$ category $\mathcal{A}$, the functor

$$
\operatorname{rep}(-, \mathcal{A}): \mathrm{Hmo}^{\text {op }} \longrightarrow \mathrm{Hmo}
$$

sends homotopy colimits to homotopy limits. Since by construction homotopy pullbacks in Hmo commute with filtered homotopy colimits, we conclude that the class of objects in Hmo which satisfy condition (3) is also stable under homotopy pushouts. Therefore, it is enough to verify condition (3) for the domains and codomains of the elements of the set $I$; see Notation 2.4. If $\mathcal{B}=\emptyset$ this is clear. The case where $\mathcal{B}=\underline{k}$ was proved in [51, Lemma 2.10]. If $\mathcal{B}=\mathcal{D}(n), n \in \mathbb{Z}$, we have a natural derived Morita equivalence

$$
\underline{k} \amalg_{\emptyset}^{\mathbb{L}} \underline{k} \simeq \underline{k} \amalg \underline{k} \stackrel{\sim}{\longrightarrow} \mathcal{D}(n) .
$$

Therefore, it remains to prove the case where $\mathcal{B}=\mathcal{S}(n), n \in \mathbb{Z}$. Since by Proposition 2.24 the derived tensor product preserves homotopy colimits, this case follows automatically from Proposition 3.1. 
Theorem 3.4. - Let $\mathcal{B}_{1}$ and $\mathcal{B}_{2}$ be homotopically finitely presented $d g$ categories. Then $\mathcal{B}_{1} \otimes^{\mathbb{L}} \mathcal{B}_{2}$ is a homotopically finitely presented $d g$ category.

Proof. - Let $\left\{\mathcal{A}_{j}\right\}_{j \in J}$ be a filtered system of dg categories. The proof is a consequence of the following weak equivalences :

$$
\begin{aligned}
\operatorname{Map}\left(\mathcal{B}_{1} \otimes^{\mathbb{L}} \mathcal{B}_{2}, \underset{j \in J}{\operatorname{hocolim}} \mathcal{A}_{j}\right) & \simeq \operatorname{Map}\left(\mathcal{B}_{1}, \operatorname{rep}\left(\mathcal{B}_{2}, \underset{j \in J}{\operatorname{hocolim}} \mathcal{A}_{j}\right)\right) \\
& \simeq \operatorname{Map}\left(\mathcal{B}_{1}, \underset{j \in J}{\operatorname{hocolim} \operatorname{rep}}\left(\mathcal{B}_{2}, \mathcal{A}_{j}\right)\right) \\
& \simeq \underset{j \in J}{\operatorname{hocolim}} \operatorname{Map}\left(\mathcal{B}_{1}, \operatorname{rep}\left(\mathcal{B}_{2}, \mathcal{A}_{j}\right)\right) \\
& \simeq \underset{j \in J}{\operatorname{hocolim}} \operatorname{Map}\left(\mathcal{B}_{1} \otimes^{\mathbb{L}} \mathcal{B}_{2}, \mathcal{A}_{j}\right) .
\end{aligned}
$$

Equivalences (3.0.8) and (3.0.11) follow from Corollary 2.17, Equivalence (3.0.9) follows from Theorem 3.3, and Equivalence (3.0.10) follows from Definition 3.2.

\section{Saturated dg categories}

In this section we introduce Kontsevich's notion of saturated dg category. Following Toën's work, we characterize these dg categories as the dualizable objects in the Morita homotopy category; see Theorem 4.8. This conceptual characterization will play an important role in our applications; see Section 8.

Definition 4.1 (Kontsevich). - ([33, 35, 36])

- A small dg category $\mathcal{A}$ is called smooth if the right $\operatorname{dg}\left(\mathcal{A}^{\mathrm{op}} \otimes \mathbb{L} \mathcal{A}\right)$-module

$$
\mathcal{A}(-,-): \mathcal{A} \otimes^{\mathbb{L}} \mathcal{A}^{\mathrm{op}} \longrightarrow \mathcal{C}_{\mathrm{dg}}(k) \quad(x, y) \mapsto \mathcal{A}(y, x)
$$

is perfect; see Definition 2.13.

- A small dg category $\mathcal{A}$ is called proper if for each ordered pair of objects $(x, y)$ in $\mathcal{A}$, the complex of $k$-modules $\mathcal{A}(x, y)$ is perfect.

- A small dg category $\mathcal{A}$ is called saturated if it is smooth and proper.

Remark 4.2. - Given a $\operatorname{dg}$ functor $F: \mathcal{B} \rightarrow \mathcal{A}$, we have a natural $\left(\mathcal{B}^{\circ \mathrm{p}} \otimes^{\mathbb{L}} \mathcal{A}\right)$ module

$$
\mathcal{B} \otimes^{\mathbb{L}} \mathcal{A}^{\text {op }} \longrightarrow \mathcal{C}_{\mathrm{dg}}(k) \quad(x, y) \mapsto \mathcal{A}(y, F x),
$$

which belongs to $\operatorname{rep}(\mathcal{B}, \mathcal{A})$ (see Definition 2.13). The above $\left(\mathcal{A}^{\mathrm{op}} \otimes^{\mathbb{L}} \mathcal{A}\right)$-module $\mathcal{A}(-,-)$ (4.0.12) is obtained by taking $\mathcal{B}=\mathcal{A}$ and $F$ the identity dg functor.

Notation 4.3. - Note that the class of smooth (resp. proper) dg categories is invariant under derived Morita equivalences (see Definition 2.8). We denote by $\mathrm{Hmo}_{\text {sat }}$ the full subcategory of Hmo (see Notation 2.10) whose objects are the saturated dg categories. 
Example 4.4. - Consider the dg categories $\mathcal{S}(n), n \geq 0$, from $§ 2.1($ ii). By construction these dg categories are proper. Thanks to Proposition 3.1, we have a natural isomorphism $\mathcal{S}(n)^{\text {op }} \otimes^{\mathbb{L}} \mathcal{S}(n) \simeq \operatorname{rep}(\mathcal{S}(n), \mathcal{S}(n))$ in Hmo. Since the $\left(\mathcal{S}(n)^{\text {op }} \otimes^{\mathbb{L}} \mathcal{S}(n)\right)$ module $\mathcal{S}(n)(-,-)$ belongs to $\operatorname{rep}(\mathcal{S}(n), \mathcal{S}(n))$ (see Remark 4.2) and we have a natural derived Morita equivalence $\mathcal{S}(n)^{\mathrm{op}} \otimes^{\mathbb{L}} \mathcal{S}(n) \rightarrow \operatorname{perf}\left(\mathcal{S}(n)^{\mathrm{op}} \otimes^{\mathbb{L}} \mathcal{S}(n)\right.$ ) (see Remark 2.14), we conclude that the $\mathrm{dg}$ categories $\mathcal{S}(n)$ are also smooth. Therefore, they are saturated.

Example 4.5. - (i) Let $X$ be a quasi-compact and separated $k$-scheme. Consider the category $\mathcal{C}(Q \operatorname{Coh}(X))$ of (unbounded) complexes of quasi-coherent sheaves on $X$. Thanks to [26], $\mathcal{C}(Q C o h(X))$ is a model category with monomorphisms as cofibrations and quasi-isomorphisms as weak equivalences. Moreover, when endowed with its natural $\mathcal{C}(k)$-enrichment, $\mathcal{C}(Q \operatorname{Coh}(X))$ becomes a $\mathcal{C}(k)$-model category in the sense of [24, Definition 4.2.18]. Let $\mathcal{L}_{\text {qcoh }}(X)$ be the dg category of fibrant objects in $\mathcal{C}(Q \operatorname{Coh}(X))$. Note that $\mathrm{H}^{0}\left(\mathcal{L}_{q c o h}(X)\right)$ is naturally equivalent to the (unbounded) derived category $\mathcal{D}_{q c o h}(X)$ of quasi-coherent sheaves on $X$. Finally, let perf $(X)$ be the full dg subcategory of $\mathcal{L}_{q c o h}(X)$ whose objects are the perfect complexes. Note that $\mathrm{H}^{0}(\operatorname{perf}(X))$ is naturally equivalent to the category of compact objects in $\mathcal{D}_{q c o h}(X)$. Thanks to Toën (see [51, Lemma 3.27]), when $X$ is a smooth and proper $k$-scheme, $\operatorname{perf}(X)$ is a saturated dg category.

(ii) Let $A$ be a $k$-algebra, which is projective of finite rank as a $k$-module, and of finite global cohomological dimension. Then, the dg category of perfect complexes of $A$-modules is a saturated dg category.

(iii) For examples coming from deformation quantization, we invite the reader to consult [33].

Definition 4.6. - Let $\mathcal{C}$ be a symmetric monoidal category with monoidal product $\otimes$ and unit object 1 . An object $X$ in $\mathcal{C}$ is called dualizable (or rigid) if there exists an object $X^{\vee}$ in $\mathcal{C}$, and maps ev $: X \otimes X^{\vee} \rightarrow \mathbf{1}$ and $\delta: \mathbf{1} \rightarrow X^{\vee} \otimes X$ such that the composites

$$
X \simeq X \otimes 1 \stackrel{\mathrm{id} \otimes \delta}{\longrightarrow} X \otimes X^{\vee} \otimes X \stackrel{\mathrm{ev} \otimes \mathrm{id}}{\longrightarrow} 1 \otimes X \simeq X
$$

and

$$
X^{\vee} \simeq \mathbf{1} \otimes X^{\vee} \stackrel{\delta \otimes \text { id }}{\longrightarrow} X^{\vee} \otimes X \otimes X^{\vee} \stackrel{\text { id } \otimes \text { ev }}{\longrightarrow} X^{\vee} \otimes \mathbf{1} \simeq X^{\vee}
$$

are identities. The object $X^{\vee}$ is called the dual of $X$, the map ev is called the evaluation map, and the map $\delta$ is called the co-evaluation map.

Remark 4.7. - (i) Given $\left(\left(X^{\vee}\right)_{1}, \mathrm{ev}_{1}, \delta_{1}\right)$ and $\left(\left(X^{\vee}\right)_{2}, \mathrm{ev}_{2}, \delta_{2}\right)$ as in Definition 4.6 , there is a unique isomorphism $\left(X^{\vee}\right)_{1} \stackrel{\sim}{\rightarrow}\left(X^{\vee}\right)_{2}$ making the natural diagrams commute. Therefore, the dual of $X$, together with the evaluation and the co-evaluation maps, is well-defined up to unique isomorphism. 
(ii) Let $X$ be a dualizable object in $\mathcal{C}$. Thanks to Equations (4.0.13) and (4.0.14), the evaluation and co-evaluation maps give rise to an adjunction:

$$
-\left.\left.\otimes X\right|_{\mathcal{C}} ^{\mathcal{C}}\right|^{\vee} \otimes-
$$

In fact, an object $X$ of $\mathcal{C}$ is dualizable if and only if there exists an object $X^{\prime}$, together with a functorial isomorphism

$$
\operatorname{Hom}_{\mathcal{C}}(Y \otimes X, Z) \simeq \operatorname{Hom}_{\mathcal{C}}\left(Y, X^{\prime} \otimes Z\right)
$$

for any objects $Y$ and $Z$ in $\mathcal{C}$ (and of course such an $X^{\prime}$ is a dual of $X$ ). In other words, $X$ is dualizable with dual $X^{\vee}$ if and only if, for any object $Z$ of $\mathcal{C}$, $X^{\vee} \otimes Z$ is the internal Hom object from $X$ to $Z$.

(iii) Let $X$ and $Y$ be dualizable objects in $\mathcal{C}$. Then $X \otimes Y$ is also a dualizable object with dual $Y^{\vee} \otimes X^{\vee}$.

(iv) Let $F: \mathcal{C} \rightarrow \mathcal{C}^{\prime}$ be a symmetric monoidal functor. Then, if $X$ is a dualizable object in $\mathcal{C}, F(X)$ is a dualizable object in $\mathcal{C}^{\prime}$ with dual $F\left(X^{\vee}\right)$

We are now ready to state the following folklore result:

Theorem 4.8. - The dualizable objects in the Morita homotopy category Hmo (see §2.4) are the saturated $d g$ categories. Moreover, the dual of a saturated dg category $\mathcal{A}$ is its opposite dg category $\mathcal{A}^{\text {op }}$.

Proof. - Let us start by introducing the category DGCAT. Its objects are the small $\operatorname{dg}$ categories. Given small $\mathrm{dg}$ categories $\mathcal{B}$ and $\mathcal{A}$, the set of morphisms from $\mathcal{B}$ to $\mathcal{A}$ is the set of isomorphism classes in $\mathcal{D}\left(\mathcal{B}^{\mathrm{op}} \otimes^{\mathbb{L}} \mathcal{A}\right)$. Given small dg categories $\mathcal{A}_{1}, \mathcal{A}_{2}$, and $\mathcal{A}_{3}$, the composition corresponds to the derived tensor product of bimodules:

$$
\begin{array}{ccc}
\operatorname{Iso} \mathcal{D}\left(\mathcal{A}_{1}^{\text {op }} \otimes^{\mathbb{L}} \mathcal{A}_{2}\right) \times \operatorname{Iso} \mathcal{D}\left(\mathcal{A}_{2}^{\text {op }} \otimes^{\mathbb{L}} \mathcal{A}_{3}\right) & \longrightarrow & \operatorname{Iso} \mathcal{D}\left(\mathcal{A}_{1}^{\text {op }} \otimes^{\mathbb{L}} \mathcal{A}_{3}\right) \\
([X],[Y]) & \mapsto & {\left[X \otimes_{\mathcal{A}_{2}}^{\mathbb{L}} Y\right] .}
\end{array}
$$

As in the case of $\mathrm{Hmo}$, the derived tensor product of dg categories gives rise to a symmetric monoidal structure on DGCAT, with unit object the dg category $\underline{k}$. The key property of DGCAT is that all its objects are dualizable: given a small dg category $\mathcal{A}$, take for dual its opposite $\mathrm{dg}$ category $\mathcal{A}^{\mathrm{op}}$, for evaluation map the isomorphism class in $\mathcal{D}\left(\left(\mathcal{A} \otimes^{\mathbb{L}} \mathcal{A}^{\mathrm{op}}\right)^{\mathrm{op}} \otimes^{\mathbb{L}} k\right) \simeq \mathcal{D}\left(\mathcal{A}^{\mathrm{op}} \otimes \mathcal{A}\right)$ of the $\left(\mathcal{A}^{\mathrm{op}} \otimes^{\mathbb{L}} \mathcal{A}\right)$-module

$$
\mathcal{A}(-,-): \mathcal{A} \otimes^{\mathbb{L}} \mathcal{A}^{\mathrm{op}} \longrightarrow \mathcal{C}_{\mathrm{dg}}(k) \quad(x, y) \mapsto \mathcal{A}(y, x),
$$

and for co-evaluation map the isomorphism class in $\mathcal{D}\left(\underline{k}^{\mathrm{op}} \otimes^{\mathbb{L}}\left(\mathcal{A}^{\mathrm{op}} \otimes^{\mathbb{L}} \mathcal{A}\right)\right) \simeq \mathcal{D}\left(\mathcal{A}^{\mathrm{op}} \otimes\right.$ $\mathcal{A})$ of the same $\left(\mathcal{A}^{\text {op }} \otimes^{\mathbb{L}} \mathcal{A}\right)$-module (4.0.16). With this choices, both composites (4.0.13) and (4.0.14) are identities. Note also that, by construction of DGCAT, and by Theorem 2.15, we have a natural symmetric monoidal functor

$$
\mathrm{Hmo} \longrightarrow \mathrm{DGCAT} \quad \mathcal{A} \mapsto \mathcal{A},
$$


which is faithful but not full.

Let $\mathcal{A}$ be a dualizable object in Hmo, with dual $\mathcal{A}^{\vee}$, evaluation map ev : $\mathcal{A} \otimes^{\mathbb{L}} \mathcal{A}^{\vee} \rightarrow$ $\underline{k}$ and co-evaluation map $\delta: \underline{k} \rightarrow \mathcal{A}^{\vee} \otimes^{\mathbb{L}} \mathcal{A}$. Since the above functor (4.0.17) is symmetric monoidal, Remark 4.7(iv) implies that $\mathcal{A}$ is dualizable in DGCAT. By unicity of duals (see Remark 4.7(i)), $\mathcal{A}^{\vee}$ is the opposite dg category $\mathcal{A}^{\mathrm{op}}$, and ev and $\delta$ are the isomorphism class in $\mathcal{D}\left(\mathcal{A}^{\mathrm{op}} \otimes^{\mathbb{L}} \mathcal{A}\right)$ of the above $\left(\mathcal{A}^{\mathrm{op}} \otimes^{\mathbb{L}} \mathcal{A}\right)$-module $\mathcal{A}(-,-)$ (see 4.0.16). The morphism ev belongs to Hmo, and so $\mathcal{A}(-,-)$ takes values in perfect complexes of $k$-modules. We conclude then that $\mathcal{A}$ is proper. Similarly, since $\delta$ is a morphism in $\mathrm{Hmo}, \mathcal{A}(-,-)$ belongs to $\operatorname{perf}\left(\mathcal{A}^{\mathrm{op}} \otimes^{\mathbb{L}} \mathcal{A}\right)$. In this case we conclude that $\mathcal{A}$ is smooth. In sum, we have shown that $\mathcal{A}$ is saturated and that $\mathcal{A}^{\vee}=\mathcal{A}^{\mathrm{op}}$.

Now, let $\mathcal{A}$ be a saturated dg category. Since $\mathcal{A}$ is proper $\mathcal{A}(-,-)$ takes values in perfect complexes of $k$-modules. Similarly, since $\mathcal{A}$ is smooth $\mathcal{A}(-,-)$ belongs to $\operatorname{perf}\left(\mathcal{A}^{\mathrm{op}} \otimes^{\mathbb{L}} \mathcal{A}\right)$. We conclude that the evaluation and co-evaluation maps of $\mathcal{A}$ in DGCAT belong to the subcategory Hmo. This implies that $\mathcal{A}$ is dualizable in $\mathrm{Hmo}$. $\sqrt{ }$

We finish this section with a comparison between saturated and homotopically finitely presented dg categories (see §3).

Lemma 4.9. - Let $\mathcal{B}$ be a saturated $d g$ category. Then, for every $d g$ category $\mathcal{A}$, we have a functorial isomorphism in $\mathrm{Hmo}$

$$
\mathcal{B}^{\mathrm{op}} \otimes^{\mathbb{L}} \mathcal{A} \simeq \operatorname{rep}(\mathcal{B}, \mathcal{A}) .
$$

Proof. - Since $\mathcal{B}^{\vee}=\mathcal{B}^{\text {op }}$, the general theory of dualizable objects implies the claim; see Remark 4.7 (iii).

Proposition 4.10. - Every saturated $d g$ category is homotopically finitely presented.

Proof. - Let $\mathcal{B}$ be a saturated dg category. Since the derived tensor product of dg categories preserves homotopy colimits in each variable (see Proposition 2.24), Lemma 4.9 implies that the functor $\operatorname{rep}(\mathcal{B},-)$ commutes with homotopy colimits. Using Theorem 3.3(3) the proof is achieved.

\section{Simplicial presheaves}

In this section we present a general theory of symmetric monoidal structures on simplicial presheaves. These general results will be used in the construction of an explicit symmetric monoidal structure on the category of non-commutative motives; see Section 7.

Starting from a small symmetric monoidal category $\mathcal{C}$, we recall that the tensor product of $\mathcal{C}$ extends to simplicial presheaves on $\mathcal{C}$; see $\S 5.4$. Then, we prove that this symmetric monoidal structure is compatible with the projective model structure; see Theorem 5.2. Finally, we study its behavior under left Bousfield localizations. 
We describe in particular a "minimal" compatibility condition between the tensor product and a localizing set; see Theorem 5.7.

5.1. Notations. - Throughout this section $\mathcal{C}$ will denote a (fixed) small category.

(i) We denote by $\widehat{\mathcal{C}}$ the category of presheaves of sets on $\mathcal{C}$, i.e. the category of contravariant functors from $\mathcal{C}$ to Set.

(ii) Given an object $\alpha$ in $\mathcal{C}$, we still denote by $\alpha$ the presheaf represented by $\alpha$

$$
\alpha: \mathcal{C}^{\mathrm{op}} \longrightarrow \text { Set }, \quad \beta \mapsto \operatorname{Hom}_{\mathcal{C}}(\beta, \alpha)
$$

(i.e. we consider the Yoneda embedding as a full inclusion).

(iii) Let $\Delta$ be the category of simplices, i.e. the full subcategory of the category of ordered sets spanned by the sets $\Delta[n]=\{0, \ldots, n\}$ for $n \geq 0$. We set

$$
\text { sSet }=\widehat{\Delta} \text {. }
$$

(iv) We denote by $s \widehat{\mathcal{C}} \simeq \widehat{\triangle \times \mathcal{C}}$ the category of simplicial objects in $\widehat{\mathcal{C}}$, i.e. the category of contravariant functors from $\Delta$ to $\widehat{\mathcal{C}}$.

(v) Finally, we consider $\widehat{\mathcal{C}}$ as a full subcategory of s $\widehat{\mathcal{C}}$. A presheaf of sets on $\mathcal{C}$ is identified with a simplicially constant object of $s \widehat{\mathcal{C}}$.

5.2. Simplicial structure. - Recall that we have a bifunctor

$$
-\otimes-: \widehat{\mathcal{C}} \times \text { Set } \longrightarrow \widehat{\mathcal{C}}
$$

defined by

$$
X \otimes K=\coprod_{k \in K} X .
$$

This defines an action of the category Set on $\widehat{\mathcal{C}}$. This construction extends to simplicial objects

$$
\mathrm{s} \widehat{\mathcal{C}} \times \mathrm{sSet} \longrightarrow \mathrm{s} \widehat{\mathcal{C}} \quad(F, K) \mapsto F \otimes K,
$$

where for $n \geq 0$ :

$$
(F \otimes K)_{n}=F_{n} \otimes K_{n} .
$$

This makes $\mathrm{s} \widehat{\mathcal{C}}$ into a simplicial category; see for instance [20, §II Definition 2.1].

5.3. Quillen Model structure. - The generating cofibrations of the classical cofibrantly generated Quillen model structure on sSet are the boundary inclusions

$$
i_{n}: \partial \Delta[n] \longrightarrow \Delta[n] \quad n \geq 0
$$

and the generating trivial cofibrations are the horn inclusions

$$
j_{n}^{k}: \Lambda[k, n] \longrightarrow \Delta[n] \quad 0 \leq k \leq n, n \geq 1 .
$$

We have the projective model structure on $s \widehat{\mathcal{C}}$ : the weak equivalences are the termwise simplicial weak equivalences, and the fibrations are the termwise Kan fibrations; see for instance [6, page 314] or [23, Theorem 11.6.1]. The projective model structure 
is proper and cellular/combinatorial. In particular, it is cofibrantly generated, with generating cofibrations

$$
1_{\alpha} \otimes i_{n}: \alpha \otimes \partial \Delta[n] \longrightarrow \alpha \otimes \Delta[n], \quad \alpha \in \mathcal{C}, n \geq 0,
$$

and generating trivial cofibrations

$$
1_{\alpha} \otimes j_{n}^{k}: \alpha \otimes \Lambda[k, n] \longrightarrow \alpha \otimes \Delta[n], \quad \alpha \in \mathcal{C}, 0 \leq k \leq n, n \geq 1 .
$$

In particular, observe that representable presheaves are cofibrant in $s \widehat{\mathcal{C}}$.

5.4. Day's convolution product. - Throughout this subsection, and until the end of Section 5, we will assume that our (fixed) small category $\mathcal{C}$ carries a symmetric monoidal structure, with tensor product $\otimes$ and unit object 1 . Under this assumption, the general theory of left Kan extensions in categories of presheaves implies formally that $\widehat{\mathcal{C}}$ is endowed with a unique closed symmetric monoidal structure which makes the Yoneda embedding a symmetric monoidal functor. We will also denote by $\otimes$ the corresponding tensor product on $\widehat{\mathcal{C}}$; the reader who enjoys explicit formulas is invited to consult $[13, \S 3]$.

This monoidal structure extends to the category $s \widehat{\mathcal{C}}$ in an obvious way: given two simplicial presheaves $F$ and $G$ on $\mathcal{C}$, we define $F \otimes G$ by the formula

$$
(F \otimes G)_{n}=F_{n} \otimes G_{n}, \quad n \geq 0 .
$$

The functor

$$
\mathrm{sSet} \longrightarrow \mathrm{s} \widehat{\mathcal{C}}, \quad K \longmapsto \mathbf{1} \otimes K
$$

is naturally endowed with a structure of symmetric monoidal functor (where sSet is considered as a symmetric monoidal category with the cartesian product as tensor product).

Definition 5.1 ([24, Definition 4.2.1] ). - Given maps $f: X \rightarrow Y$ and $g: A \rightarrow B$ in a symmetric monoidal category (with tensor product $\otimes$ ), the pushout product map $f \square g$ of $f$ and $g$ is given by:

$$
f \square g: X \otimes B \coprod_{X \otimes A} Y \otimes A \longrightarrow Y \otimes B .
$$

Theorem 5.2. - The category $\mathrm{s} \widehat{\mathcal{C}}$, endowed with the projective model category structure is a symmetric monoidal model category (see [24, Definition 4.2.6]).

Proof. - As the model category of simplicial sets is a symmetric monoidal model category (with the cartesian product as tensor product), the result follows from the explicit description of the generating cofibrations and generating trivial cofibrations of $\mathrm{s} \widehat{\mathcal{C}}$ : given two objects $\alpha$ and $\alpha^{\prime}$ in $\mathcal{C}$ and two maps $i: K \rightarrow L$ and $i^{\prime}: K^{\prime} \rightarrow L^{\prime}$ in sSet, we have

$$
\left(1_{\alpha} \otimes i\right) \square\left(1_{\alpha^{\prime}} \otimes i^{\prime}\right) \simeq 1_{\alpha \otimes \alpha^{\prime}} \otimes\left(i \square i^{\prime}\right) .
$$


Since for any object $\alpha$ in $\mathcal{C}$, the functor $K \mapsto \alpha \otimes K$ is a left Quillen functor from sSet to $\mathrm{s} \widehat{\mathcal{C}}$, the proof is finished.

Let $s \widehat{\mathcal{C}}$. be the category of pointed simplicial presheaves on $\mathcal{C}$. The forgetful functor $U: \mathrm{s} \widehat{\mathcal{C}} \bullet \rightarrow s \widehat{\mathcal{C}}$ has a left adjoint

$$
\mathrm{s} \widehat{\mathcal{C}} \longrightarrow \mathrm{s} \widehat{\mathcal{C}} \bullet, \quad F \longmapsto F_{+},
$$

where $F_{+}$denotes the pointed simplicial presheaf $F \amalg \star$, with $\star$ the terminal object of $\mathrm{s} \widehat{\mathcal{C}}$. The category $s \widehat{\mathcal{C}}$. is then canonically a cofibrantly generated model category, in such a way that the functor (5.4.1) is a left Quillen functor; see [24, Proposition 1.1.8 and Lemma 2.1.21].

Furthermore, there is a unique symmetric monoidal structure on $s \widehat{\mathcal{C}} \bullet$ making the functor (5.4.1) symmetric monoidal. The unit object is $\mathbf{1}_{+}$, and the tensor product Q. is defined as follows: for two pointed simplicial presheaves $F$ and $G$, their tensor product is given by the following pushout in the category $s \widehat{\mathcal{C}}$ of unpointed simplicial presheaves :

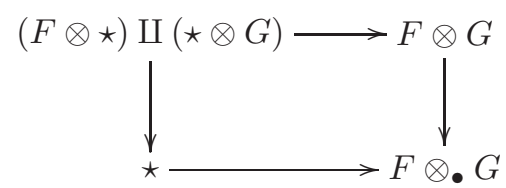

In particular, for two simplicial presheaves $F$ and $G$, we have

$$
(F \otimes G)_{+} \simeq F_{+} \otimes_{\bullet} G_{+} .
$$

Proposition 5.3. - With the above definition, the model category $\mathrm{s} \widehat{\mathcal{C}}$. is a symmetric monoidal model category.

Proof. - The generating cofibrations (resp. generating trivial cofibrations) of $\mathrm{s} \widehat{\mathcal{C}}$. are the maps of shape $A_{+} \rightarrow B_{+}$for $A \rightarrow B$ a generating cofibration (resp. generating trivial cofibration) of $s \widehat{\mathcal{C}}$. As the functor (5.4.1) is a left Quillen functor, the result follows immediately from Formula (5.4.2) and from Theorem 5.2.

Remark 5.4. - In practice, we shall refer to the pointed tensor product $\otimes_{\bullet}$ as the canonical tensor product on $s \widehat{\mathcal{C}} \bullet$ associated to the monoidal structure on $\mathcal{C}$. Whenever there is no ambiguity, we denote the pointed tensor product simply by $\otimes$.

\subsection{Left Bousfield localization. -}

Definition 5.5. - ([23, Definition 3.1.4]) Let $\mathcal{M}$ be a model category and $S$ a set of morphisms in $\mathcal{M}$. An object $X$ in $\mathcal{M}$ is called $S$-local if it is fibrant and for every element $s: A \rightarrow B$ of the set $S$, the induced map of homotopy function complexes

$$
s^{*}: \operatorname{Map}(B, X) \longrightarrow \operatorname{Map}(A, X)
$$


is a weak equivalence. A map $g: X \rightarrow Y$ in $\mathcal{M}$ is called a $S$-local equivalence if for every $S$-local object $W$, the induced map of homotopy function complexes

$$
g^{*}: \operatorname{Map}(Y, W) \longrightarrow \operatorname{Map}(X, W)
$$

is a weak equivalence

Recall that, if $\mathcal{M}$ is cellular (or combinatorial) and left proper, the left Bousfield localization of $\mathcal{M}$ is the model category $\mathrm{L}_{S} \mathcal{M}$ whose underlying category is $\mathcal{M}$, whose cofibrations are those of $\mathcal{M}$, and whose weak equivalences are the $S$-local weak equivalences; see [23, Definition 9.3.1(1)]. The fibrant objects of $L_{S} \mathcal{M}$ are then the objects of $\mathcal{M}$ which are both fibrant and $S$-local, and the fibrations between fibrant objects in $\mathrm{L}_{S} \mathcal{M}$ are the fibrations of $\mathcal{M}$.

Proposition 5.6. - Let $\mathcal{M}$ be a left proper, cellular (or combinatorial), symmetric monoidal model category (with tensor product $\otimes$ ), and $S$ a set of morphisms in $\mathcal{M}$. Assume that the following conditions hold:

(i) $\mathcal{M}$ admits generating sets of cofibrations and of trivial cofibrations consisting of maps between cofibrant objects;

(ii) every element of $S$ is a map between cofibrant objects;

(iii) given a cofibrant object $X$, the functor $X \otimes(-)$ sends the element of $S$ to $S$-local weak equivalences.

(iv) the unit object of the monoidal structure on $\mathcal{M}$ is cofibrant.

Then the left Bousfield localization $\mathrm{L}_{S} \mathcal{M}$ of $\mathcal{M}$ with respect to the set $S$ is a symmetric monoidal model category.

Proof. - The left Bousfield localization $\mathrm{L}_{S} \mathcal{M}$ of $\mathcal{M}$ with respect to the set $S$ is cofibrantly generated (see [23, Theorem 4.1.1(3)]) and thanks to condition (iv) the unit object is cofibrant. Therefore, by [24, Lemma 4.2.7] it is enough to verify the pushout product axiom on the sets of generating (trivial) cofibrations. The class of cofibrations in $\mathrm{L}_{S} \mathcal{M}$ and in $\mathcal{M}$ is the same, and so half of the pushout product axiom is automatically verified. Now, let $g: A \longrightarrow B$ be a generating cofibration in $\mathrm{L}_{S} \mathcal{M}$ and $f: X \stackrel{\sim}{\longrightarrow} Y$ a generating trivial cofibration in $\mathrm{L}_{S} \mathcal{M}$. By condition (i), we may assume, that the objects $X, Y, A$ and $B$ are cofibrant. Moreover, condition (iii) implies that tensoring by a cofibrant object preserves $S$-local weak equivalences. Consider the following commutative diagram:

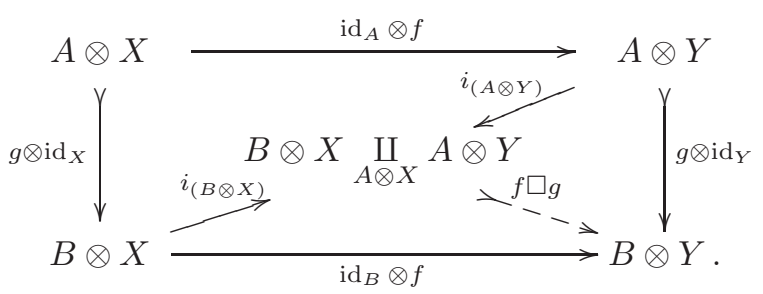


Using the two-out-of-three property for $S$-local weak equivalences, we conclude that $f \square g$ is an $S$-local equivalence.

Theorem 5.7. - Let $S$ be a set of morphisms between cofibrant objects in $\mathrm{s} \widehat{\mathcal{C}}$. Assume that the following condition holds:

(C) given an object $\alpha$ in $\mathcal{C}$ and a map $G \rightarrow H$ in $S$, the morphism $\alpha \otimes G \rightarrow \alpha \otimes H$ is an $S$-local equivalence.

Then the left Bousfield localization $\mathrm{L}_{S} \mathrm{~s} \widehat{\mathcal{C}}$ of $\mathrm{s} \widehat{\mathcal{C}}$ with respect to the set $S$, is a symmetric monoidal model category.

Proof. - We shall apply Proposition 5.6. It is sufficient to prove condition (iii) of loc. cit. In other words, we need to prove that for any object $F$ of $\mathrm{s} \widehat{\mathcal{C}}$ and any map $G \rightarrow H$ in $S$, the map

$$
F \otimes^{\mathbb{L}} G \longrightarrow F \otimes^{\mathbb{L}} H
$$

in $\mathrm{Ho}(\mathrm{s} \widehat{\mathcal{C}})$ is sent to an isomorphism in $\mathrm{Ho}\left(L_{S} s \widehat{\mathcal{C}}\right)$. Thanks to Condition (C) this is the case when $F$ is representable. As the functors $(-) \otimes^{\mathbb{L}} F$ commute with homotopy colimits, the general case follows from the fact that the functor $\mathrm{Ho}(\mathrm{s} \widehat{\mathcal{C}}) \rightarrow \mathrm{Ho}\left(\mathrm{L}_{S} \mathrm{~s} \widehat{\mathcal{C}}\right)$ commutes with homotopy colimits, and that any simplicial presheaf is a homotopy colimit of representable presheaves (see for instance [15, Proposition 2.9] or [8, Proposition 3.4.34]).

Corollary 5.8. - Assume that $S$ is a set of maps in $\mathcal{C}$ which is closed under tensor product in $\mathcal{C}$ (up to isomorphism). Then, by considering $S$ as a set of maps in $\widehat{\mathbf{C}}$ via the Yoneda embedding, the left Bousfield localization $\mathrm{L}_{S} \mathrm{~s} \widehat{\mathcal{C}}$ is a symmetric monoidal model category.

Proof. — Condition (C) of the Theorem 5.7 is trivially satisfied.

\section{Monoidal stabilization}

In this section we relate the general theory of spectra with the general theory of symmetric spectra; see Theorem 6.1. This will be used in the construction of an explicit symmetric monoidal structure on the category of non-commutative motives; see Section 7.

Let $\mathcal{M}$ be a cellular (or combinatorial) pointed simplicial left proper model category. There is then a natural action of the category of pointed simplicial sets

$$
\mathcal{M} \times \mathrm{sSet}_{\bullet} \longrightarrow \mathcal{M}, \quad(X, K) \longmapsto X \otimes K
$$

(in the literature, $X \otimes K$ is usually denoted by $X \wedge K$ ). We denote by $\operatorname{Sp}^{\mathbb{N}}(\mathcal{M})$ the stable model category of $S^{1}$-spectra on $\mathcal{M}$, where $S^{1}=\Delta[1] / \partial \Delta[1]$ is the simplicial circle, seen as endofunctor $X \mapsto X \otimes S^{1}$ of $\mathcal{M}$; see [25, §1].

Assume that $\mathcal{M}$ is a symmetric monoidal model category with cofibrant unit object 1. We write $\operatorname{Sp}^{\Sigma}(\mathcal{M})$ for the stable symmetric monoidal model category of symmetric 
$S^{1} \otimes 1$-spectra on $\mathcal{M}$; see $[25, \S 7]$. In this situation we have a symmetric monoidal left Quillen functor

$$
\Sigma^{\infty}: \mathcal{M} \longrightarrow \operatorname{Sp}^{\Sigma}(\mathcal{M}) .
$$

Theorem 6.1. - Under the above assumptions, the model categories $\operatorname{Sp}^{\mathbb{N}}(\mathcal{M})$ and $\mathrm{Sp}^{\Sigma}(\mathcal{M})$ are canonically Quillen equivalent.

Proof. - By applying [25, Theorem 10.3 and Corollary 10.4], it is sufficient to prove that $S^{1} \otimes \mathbf{1}$ is symmetric in $\mathrm{Ho}(\mathcal{M})$, i.e. that the permutation $(1,2,3)$ acts trivially on $\left(S^{1} \otimes \mathbf{1}\right)^{\otimes 3}$. Using [9, Corollaire 6.8], we see that $\left(S^{1} \otimes \mathbf{1}\right)^{\otimes 3} \simeq S^{3} \otimes \mathbf{1}$ in $\mathrm{Ho}(\mathcal{M})$, and that it is sufficient to check this condition in the case where $\mathcal{M}$ is the model category of pointed simplicial sets. Finally, in this particular case, the result is well known; see for instance [24, Lemma 6.6.2].

\section{Symmetric monoidal structure}

In this section we motivate, state and prove our main result: the localizing motivator carries a canonical symmetric monoidal structure; see Theorem 7.5.

Definition 7.1. - A sequence of triangulated categories

$$
\mathcal{R} \stackrel{I}{\longrightarrow} \mathcal{S} \stackrel{P}{\longrightarrow} \mathcal{T}
$$

is called exact if the composition is zero, the functor $I$ is fully-faithful and the induced functor from the Verdier quotient $\mathcal{S} / \mathcal{R}$ to $\mathcal{T}$ is cofinal, i.e. it is fully-faithful and every object in $\mathcal{T}$ is a direct summand of an object of $\mathcal{S} / \mathcal{R}$; see [39, §2] for details. A sequence of dg categories

$$
\mathcal{A} \stackrel{F}{\longrightarrow} \mathcal{B} \stackrel{G}{\longrightarrow} \mathcal{C}
$$

is called exact if the induced sequence of triangulated categories (see $\S 2.2$ )

$$
\mathcal{D}(\mathcal{A}) \stackrel{\mathbb{L} F_{5}}{\longrightarrow} \mathcal{D}(\mathcal{B}) \stackrel{\mathbb{L} G_{3}}{\longrightarrow} \mathcal{D}(\mathcal{C})
$$

is exact.

Recall from $[42, \S 10]$ the construction of the universal localizing invariant.

Theorem 7.2. - ([42, Theorem 10.5]) There exists a morphism of derivators

$$
\mathcal{U}_{\mathrm{dg}}^{\text {loc }}: \mathrm{HO}(\text { dgcat }) \longrightarrow \mathrm{Mot}_{\mathrm{dg}}^{\text {loc }},
$$

with values in a strong triangulated derivator (see §A.3), which has the following properties:

flt) $\mathcal{U}_{\mathrm{dg}}^{\mathrm{loc}}$ preserves filtered homotopy colimits;

p) $\mathcal{U}_{\mathrm{dg}}^{\text {loc }}$ preserves the terminal object; 
loc) $\mathcal{U}_{\mathrm{dg}}^{\text {loc }}$ satisfies localization, i.e. sends exact sequence of $d g$ categories

$$
\mathcal{A} \longrightarrow \mathcal{B} \longrightarrow \mathcal{C}
$$

to distinguished triangles in $\operatorname{Mot}_{\mathrm{dg}}^{\mathrm{loc}}(e)$

$$
\mathcal{U}_{\mathrm{dg}}^{\mathrm{loc}}(\mathcal{A}) \longrightarrow \mathcal{U}_{\mathrm{dg}}^{\mathrm{loc}}(\mathcal{B}) \longrightarrow \mathcal{U}_{\mathrm{dg}}^{\mathrm{loc}}(\mathcal{C}) \longrightarrow \mathcal{U}_{\mathrm{dg}}^{\mathrm{loc}}(\mathcal{A})[1]
$$

Moreover, $\mathcal{U}_{\mathrm{dg}}^{\mathrm{loc}}$ is universal with respect to these properties, i.e. given any strong triangulated derivator $\mathbb{D}$, we have an induced equivalence of categories

$$
\left(\mathcal{U}_{\mathrm{dg}}^{\text {loc }}\right)^{*}: \underline{\operatorname{Hom}}_{!}\left(\operatorname{Mot}_{\mathrm{dg}}^{\mathrm{loc}}, \mathbb{D}\right) \stackrel{\sim}{\longrightarrow} \underline{\operatorname{Hom}}_{\mathrm{loc}}(\mathrm{HO}(\text { dgcat }), \mathbb{D}),
$$

where the left-hand side stands for the category of homotopy colimit preserving morphisms of derivators, while the right-hand side stands for the full subcategory of Hom $(\mathrm{HO}($ dgcat $), \mathbb{D})$ spanned by the morphisms of derivators which verify the three conditions above.

Definition 7.3. - The objects of the category $\underline{\mathrm{Hom}}_{\mathrm{loc}}(\mathrm{HO}(\mathrm{dgcat}), \mathbb{D})$ are called localizing invariants and $\mathcal{U}_{\mathrm{dg}}^{\mathrm{loc}}$ is called the universal localizing invariant. Because of its universal property, which is a reminiscence of motives, Mot ${ }_{\mathrm{dg}}^{\text {loc }}$ is called the localizing motivator. Its base category $\operatorname{Mot}_{d g}^{l o c}(e)$ is a triangulated category and is morally what we would like to consider as the category of non-commutative motives.

Example 7.4. - Examples of localizing invariants include Hochschild homology and cyclic homology (see [42, Theorem 10.7]), non-connective algebraic $K$-theory (see [42, Theorem 10.9]), and even topological Hochschild homology and topological cyclic homology (see [2, Theorem 6.1] and [45, §8]).

In this section we introduce a new ingredient in Theorem 7.2: symmetric monoidal structures. As shown in Theorem 2.23 the derivator $\mathrm{HO}$ (dgcat) carries a symmetric monoidal structure. It is therefore natural to consider localizing invariants which are symmetric monoidal; see Examples 7.9-7.11. Our main result is the following.

Theorem 7.5. - The localizing motivator Mot $_{\mathrm{dg}}^{\mathrm{loc}}$ carries a canonical symmetric monoidal structure $-\otimes^{\mathbb{L}}-$, making the universal localizing invariant $\mathcal{U}_{\mathrm{dg}}^{\text {loc }}$ symmetric monoidal (see $\S A .5)$. Moreover, this tensor product preserves homotopy colimits in each variable, and is characterized by the following universal property: given any strong triangulated derivator $\mathbb{D}$ (see $\S A .3)$, endowed with a symmetric monoidal structure which preserves homotopy colimits in each variable, we have an induced equivalence of categories

$$
\left(\mathcal{U}_{\mathrm{dg}}^{\text {loc }}\right)^{*}: \underline{\operatorname{Hom}}_{!}^{\otimes}\left(\operatorname{Mot}_{\mathrm{dg}}^{\mathrm{loc}}, \mathbb{D}\right) \stackrel{\sim}{\longrightarrow} \underline{\operatorname{Hom}}_{\mathrm{loc}}^{\otimes}(\mathrm{HO}(\text { dgcat }), \mathbb{D}),
$$

where the left-hand side stands for the category of symmetric monoidal homotopy colimit preserving morphisms of derivators, while the right-hand side stands for the category of symmetric monoidal morphisms of derivators which belong to the category 


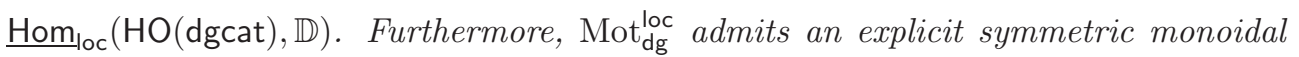
Quillen model.

Definition 7.6. - The objects of the category $\underline{\mathrm{Hom}}_{\mathrm{loc}}^{\otimes}(\mathrm{HO}(\mathrm{dgcat}), \mathbb{D})$ are called symmetric monoidal localizing invariants.

Corollary 7.7. - Any dualizable object of $\operatorname{Mot}_{\mathrm{dg}}^{\mathrm{loc}}(e)$ is compact; see Definition 4.6 and §1.2. In particular, given any saturated $d g$ category $\mathcal{A}$ (see Definition 4.1), the object $\mathcal{U}_{\mathrm{dg}}^{\mathrm{loc}}(\mathcal{A})$ is compact.

Proof. - Let $M$ be a dualizable object of $\operatorname{Mot}_{\mathrm{dg}}^{\mathrm{loc}}(e)$. We need to prove that the functor $\operatorname{Hom}(M,-)$ commutes with arbitrary sums. Since $M$ is dualizable, this functor is isomorphic to $\operatorname{Hom}\left(\mathcal{U}_{\mathrm{dg}}^{\text {loc }}(\underline{k}), M^{\vee} \otimes^{\mathbb{L}}-\right)$. The unit object $\mathcal{U}_{\mathrm{dg}}^{\text {loc }}(\underline{k})$ is known to be compact (see [11, Theorem 7.16]), and so the first assertion is proven. The second assertion follows from the fact that $\mathcal{U}_{\mathrm{dg}}^{\text {loc }}$ is symmetric monoidal, and that $\mathcal{U}_{\mathrm{dg}}^{\text {loc }}(\mathcal{A})$ is dualizable for any saturated $\mathrm{dg}$ category $\mathcal{A}$ (see Remark 4.7 (iv) and Theorem 4.8).

Remark 7.8. - Although we do not know if the triangulated category $\operatorname{Mot}_{\mathrm{dg}}^{\mathrm{loc}}(e)$ is compactly generated, Corollary 7.7 implies that the localizing triangulated subcategory of $\operatorname{Mot}_{\mathrm{dg}}^{\mathrm{loc}}(e)$ generated by dualizable objects is compactly generated.

Before proving Theorem 7.5, let us give some examples of symmetric monoidal localizing invariants.

Example 7.9 (Hochschild homology). - Let $\mathcal{A}$ be a small $k$-flat dg category; see Definition 2.19. We can associate to $\mathcal{A}$ a simplicial object in $\mathcal{C}(k)$, i.e. a contravariant functor from $\Delta$ to $\mathcal{C}(k)$ : its $n$-th term is given by

$$
\bigoplus_{\left(x_{0}, \ldots, x_{n}\right)} \mathcal{A}\left(x_{n}, x_{0}\right) \otimes \mathcal{A}\left(x_{n-1}, x_{n}\right) \otimes \cdots \otimes \mathcal{A}\left(x_{0}, x_{1}\right)
$$

where $\left(x_{0}, \ldots, x_{n}\right)$ is an ordered sequence of objects in $\mathcal{A}$. The face maps are given by

$$
d_{i}\left(f_{n}, \ldots, f_{i}, f_{i-1}, \ldots, f_{0}\right)= \begin{cases}\left(f_{n}, \ldots, f_{i} \circ f_{i-1}, \ldots, f_{0}\right) & \text { if } i>0 \\ (-1)^{(n+\sigma)}\left(f_{0} \circ f_{n}, \ldots, f_{1}\right) & \text { if } i=0\end{cases}
$$

where $\sigma=\left(\operatorname{deg} f_{0}\right)\left(\operatorname{deg} f_{1}+\ldots+\operatorname{deg} f_{n-1}\right)$, and the degenerancies maps are given by

$$
s_{j}\left(f_{n}, \ldots, f_{j}, f_{j-1}, \ldots, f_{0}\right)=\left(f_{n}, \ldots, f_{j}, \mathrm{id}_{x_{j}}, f_{j-1}, \ldots, f_{0}\right) .
$$

Associated to this simplicial object we have a chain complex in $\mathcal{C}(k)$ (by the DoldKan equivalence), and so a bicomplex of $k$-modules. The Hochschild complex $H H(\mathcal{A})$ of $\mathcal{A}$ is the sum-total complex associated to this bicomplex. If $\mathcal{A}$ is an arbitrary $\operatorname{dg}$ category, its Hochschild complex is obtained by first taking a $k$-flat (e.g. cofibrant) resolution of $\mathcal{A}$; see Definition 2.19. This construction furnishes us a functor

$$
H H: \text { dgcat } \longrightarrow \mathcal{C}(k),
$$


which by [42, Theorem 10.7] gives rise to a localizing invariant

$$
H H: \mathrm{HO}(\text { dgcat }) \longrightarrow \mathrm{HO}(\mathcal{C}(k)) \text {. }
$$

Given small dg categories $\mathcal{A}$ and $\mathcal{B}$, we have a functorial quasi-isomorphism

$$
\operatorname{sh}: H H(\mathcal{A}) \otimes H H(\mathcal{B}) \longrightarrow H H(\mathcal{A} \otimes \mathcal{B})
$$

given by the shuffle product map; see [38, §4.2.3]. The localizing invariant (7.0.1), endowed with the shuffle product map, becomes then a symmetric monoidal localizing invariant.

Example 7.10 (Mixed complexes). - Following Kassel $[29, \S 1]$ we denote by $\Lambda$ the dg algebra $k[\epsilon] / \epsilon^{2}$, where $\epsilon$ is of degree -1 and $d(\epsilon)=0$. Under this notation, a mixed complex is a right $\mathrm{dg} \Lambda$-module (see Definition 2.6).

Let $\mathcal{A}$ be a small dg category. The Hochschild complex $H H(\mathcal{A})$ of $\mathcal{A}$ (see Example 7.9), endowed with the cyclic operator

$$
t_{n}\left(f_{n-1}, \ldots, f_{0}\right)=(-1)^{n+\sigma}\left(f_{0}, f_{n-1}, f_{n-2}, \ldots, f_{1}\right),
$$

gives rise to a mixed complex $C(\mathcal{A})$; see [31, §1.3]. The assignment $\mathcal{A} \mapsto C(\mathcal{A})$ yields a localizing invariant

$$
C: \mathrm{HO}(\text { dgcat }) \longrightarrow \mathrm{HO}(\mathcal{C}(\Lambda))
$$

with values in the derivator associated to right $\mathrm{dg} \Lambda$-modules; see [42, Theorem 10.7]. Recall from $[29, \S 1]$ that the category $\mathcal{C}(\Lambda)$ carries a natural symmetric monoidal structure whose unit object is $k$ : given two mixed complexes there is a canonical mixed complex structure on the tensor product of the underlying complexes. Moreover, this symmetric monoidal structure is compatible with the projective model structure (see $\S 2.2)$. Thanks to [29, Theorem 2.4] the localizing invariant (7.0.2) becomes then a symmetric monoidal localizing invariant.

This example will be used in the construction of a canonical Chern character map from non-connective algebraic $K$-theory to negative cyclic homology; see Example 8.10.

Example 7.11 (Periodic complexes). - In this example we assume that our base ring $k$ is a field. Let $k[u]$ be the cocommutative Hopf algebra of polynomials in one variable $u$ of degree 2 ; see $[29, \S 1]$. Consider the symmetric monoidal model category $k[u]$-Comod of $k[u]$-comodules; see [24, Theorem 2.5.17]. The monoidal structure is given by the cotensor product $-\square_{k[u]}-$ of comodules, with unit $k[u]$.

Given a mixed complex $M$ (see Example 7.10) we denote by $P(M)$ the $k[u]$ comodule, whose underlying complex is $M \otimes_{\Lambda}^{\mathbb{L}} k$, obtained by iteration of the map

$$
\left(M \otimes_{\Lambda}^{\mathbb{L}} k\right)[-2] \stackrel{S}{\longrightarrow} M \otimes_{\Lambda}^{\mathbb{L}} k,
$$


see [29, Proposition 1.4]. Using [29, Theorem 1.7] and [19, Proposition 9.2], we conclude that we have a symmetric monoidal morphism of derivators

$$
P: \mathrm{HO}(\mathcal{C}(\Lambda)) \longrightarrow \mathrm{HO}(k[u] \text {-Comod }) \text {. }
$$

By composing $P$ with the localizing invariant (7.0.2) we obtain then a symmetric monoidal localizing invariant

$$
(P \circ C): \mathrm{HO}(\text { dgcat }) \longrightarrow \mathrm{HO}(k[u] \text {-Comod }) .
$$

This example will be used in the construction of a canonical Chern character map from non-connective algebraic $K$-theory to periodic cyclic homology; see Example 8.11.

7.1. Proof of Theorem 7.5. - We will use freely the theory of derivators which is recalled and developed in the appendix. Recall from $[42, \S 10]$ that $\mathcal{U}_{\mathrm{dg}}^{\mathrm{loc}}$ is obtained by the following composition:

$$
\mathrm{HO}(\text { dgcat }) \stackrel{\mathbb{R} h}{\longrightarrow} \mathrm{L}_{\Sigma} \text { Hot }_{\text {ggcat }_{f}} \stackrel{\Phi}{\longrightarrow} \mathrm{L}_{\Sigma, P} \text { Hot }_{\text {dgcat }_{f}} \stackrel{\text { stab }}{\longrightarrow} \mathrm{St}\left(\mathrm{L}_{\Sigma, P} \text { Hot }_{\text {dgcat }_{f}}\right) \stackrel{\gamma}{\longrightarrow} \text { Mot }_{\mathrm{dg}}^{\text {loc }} .
$$

The morphism stab corresponds to a stabilization procedure (see $\S \mathrm{A} .8$ ) and the morphism $\gamma$ to a left Bousfield localization procedure (see $\S$ A.7). Since these procedures commute (see Proposition A.12), we can also obtain $\mathcal{U}_{\mathrm{dg}}^{\text {loc }}$ by the following composition

$$
\mathrm{HO}(\text { dgcat }) \stackrel{\mathbb{R} h}{\rightarrow} \mathrm{L}_{\Sigma} \text { Hot }_{\text {dgcat }_{\mathrm{f}}} \stackrel{\Phi}{\longrightarrow} \mathrm{L}_{\Sigma, P} \text { Hot }_{\text {dgcat }_{\mathrm{f}}} \stackrel{\gamma}{\longrightarrow} \text { Mot }_{\mathrm{dg}}^{\text {uloc }} \stackrel{\text { stab }}{\longrightarrow} \text { Mot }_{\mathrm{dg}}^{\text {loc }},
$$

where $\operatorname{Mot}_{\mathrm{dg}}^{\mathrm{uloc}}$ is the unstable analogue of the localizing motivator.

The proof of Theorem 7.5 will consist on the concatenation of Propositions 7.127.16 followed by Remark 7.18. In each one of these propositions we construct an explicit symmetric monoidal Quillen model for the corresponding (intermediate) derivator of the composition (7.1.1).

We start by fixing on dgcat a fibrant resolution functor $R$, a cofibrant resolution functor $Q$, a left framing $\Gamma_{*}$ (i.e. a well-behaved cosimplicial resolution functor; see [24, Definition 5.2.7 and Theorem 5.2.8]), as well as a small full subcategory dgcat $_{\mathrm{f}}$ of dgcat, satisfying the following properties :

(a) any finite dg cell (see Definition 2.5) is in dgcat $_{f}$;

(b) any object in dgcat $_{\mathrm{f}}$ is homotopically finitely presentated (see Definition 3.2);

(c) given any object $\mathcal{A}$ in dgcat $_{\mathrm{f}}, Q(R(\mathcal{A}))$ and $Q(\mathcal{A})$ belong to dgcat ;

(d) for any cofibrant object $\mathcal{A}$ of $\operatorname{dgcat}_{\mathrm{f}}$, if $\Gamma_{*}(\mathcal{A})$ denotes the given cosimplicial frame of $\mathcal{A}$, then $\Gamma_{n}(\mathcal{A})$ belongs to $\operatorname{dgcat}_{\mathrm{f}}$ for all $n \geq 0$.

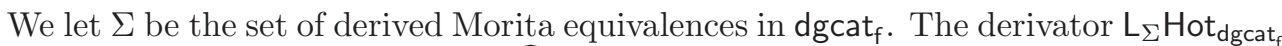
is simply the derivator $\mathrm{HO}\left(\mathrm{L}_{\Sigma} \mathrm{s} \widehat{\mathrm{dgcat}_{f}}\right)$ associated to the left Bousfield localization of the projective model structure on $\mathrm{s} \overline{\mathrm{dgcat}}_{\mathrm{f}}$ ( $\mathrm{see} \S 5.3$ ), with respect to the set $\Sigma$. Note that, up to Quillen equivalence, this construction does not depend on the choice of the category dgcat $\mathrm{f}_{\mathrm{f}}$ but only on the Dwyer-Kan localization of dgcat $_{f}$ by $\Sigma$ (see [18]). The above stability properties imply that the Dwyer-Kan localization of $\operatorname{dgcat}_{\mathrm{f}}$ by $\Sigma$ 
is simply (equivalent to) the full simplicial subcategory of the Dwyer-Kan localization of the model category dgcat spanned by the homotopically finitely presented dg categories. In order to obtain a symmetric monoidal structure, we have then the freedom to add the following properties to $\mathrm{dgcat}_{\mathrm{f}}$ :

(e) any dg category in dgcat $\mathrm{f}_{\mathrm{f}}$ is $k$-flat (see Definition 2.19);

(f) given any dg categories $\mathcal{A}$ and $\mathcal{B}$ in dgcat $_{\mathrm{f}}, \mathcal{A} \otimes \mathcal{B}$ belongs to dgcat f this makes $_{\mathrm{f}}$ sense because of Theorem 3.4).

In the sequel, we assume that a small full subcategory dgcat $_{f}$ of dgcat satisfying all the above properties (a)-(f) has been chosen; for instance, one might consider the smallest one relatively to $R, Q$ and $\Gamma_{*}$. The morphism

$$
\mathbb{R} \underline{h}: \mathrm{HO}(\text { dgcat }) \longrightarrow \mathrm{L}_{\Sigma} \mathrm{Hot}_{\text {dgcat }_{\mathrm{f}}}=\mathrm{HO}\left(\mathrm{L}_{\Sigma} \mathrm{s} \widehat{\mathrm{dgcat}}_{\mathrm{f}}\right)
$$

is induced by the functor

$$
\underline{h}: \text { dgcat } \longrightarrow \text { s } \widehat{\text { dgcat }}_{\mathrm{f}},
$$

which associates to any dg category $\mathcal{A}$ the simplicial presheaf on dgcat $_{\mathrm{f}}$ :

$$
\mathbb{R} \underline{h}(\mathcal{A}): \mathcal{B} \longmapsto \operatorname{Map}(\mathcal{B}, \mathcal{A})=\operatorname{Hom}\left(\Gamma_{*}(Q(\mathcal{B})), R(\mathcal{A})\right) .
$$

Proposition 7.12. - The tensor product of $d g$ categories in dgcat $_{f}$ extends uniquelly to a closed symmetric monoidal structure on the category of simplicial presheaves on $\mathrm{dgcat}_{\mathrm{f}}$, making $\mathrm{L}_{\Sigma} \mathrm{s} \widehat{\mathrm{dgcat}}_{\mathrm{f}}$ into a symmetric monoidal model category. As a consequence, the derivator $\mathrm{L}_{\Sigma} \mathrm{Hot}_{\mathrm{dgcat}_{\mathrm{f}}}$ carries a symmetric monoidal structure, making the morphism $\mathbb{R} \underline{h}$ symmetric monoidal. Moreover, given any derivator $\mathbb{D}$, the category of filtered homotopy colimit preserving symmetric monoidal morphisms from $\mathrm{HO}$ (dgcat) to $\mathbb{D}$ is canonically equivalent to the category of homotopy colimit preserving symmetric monoidal morphisms from $\mathrm{L}_{\Sigma} \operatorname{Hot}_{\mathrm{dgcat}_{f}}$ to $\mathbb{D}$.

Proof. - As derived Morita equivalences are stable under derived tensor product, it follows immediately from Corollary 5.8 that $L_{\Sigma} s \widehat{d g c a t}_{f}$ is a symmetric monoidal model category.

Let us now show that the morphism $\mathbb{R} \underline{h}$ is symmetric monoidal. Recall from [42, $\S 5]$ that the morphism $\mathbb{R} \underline{h}$ preserves filtered homotopy colimits and that we have a commutative diagram

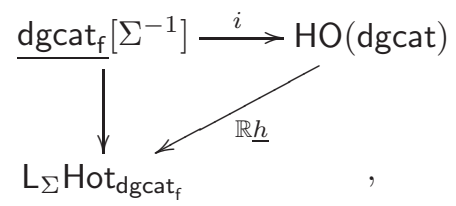

where $\operatorname{dgcat}_{f}$ stands for the prederivator represented by dgcat ${ }_{f}$, and $\operatorname{dgcat}_{f}\left[\Sigma^{-1}\right]$ for its formal localization by $\Sigma$. By construction, the left vertical morphism in the above diagram (7.1.2) is symmetric monoidal and the symmetric monoidal structure on $L_{\Sigma} \mathrm{Hot}_{\mathrm{dgcat}_{f}}$ preserves homotopy colimits in each variable. Moreover, thanks 
to Lemma 2.24, the symmetric monoidal structure on $\mathrm{HO}$ (dgcat) preserves filtered homotopy colimits.

Now, recall the universal property of $\mathrm{HO}$ (dgcat): it is the free completion of the prederivator $\operatorname{dgcat}_{\mathrm{f}}\left[\Sigma^{-1}\right]$ by filtered homotopy colimits. In other words, given any derivator $\mathbb{D}$, the category of filtered homotopy colimit preserving morphisms from $\mathrm{HO}$ (dgcat) to $\mathbb{D}$ is canonically equivalent to the category of morphisms from $\operatorname{dgcat}_{\mathrm{f}}\left[\Sigma^{-1}\right]$ to $\mathbb{D}$; see $[42, \S 5]$. Replacing $\mathbb{D}$ by the derivator of filtered homotopy colimit preserving morphisms from $\mathrm{HO}$ (dgcat) to $\mathbb{D}$, we deduce that the category of morphisms from $\mathrm{HO}($ dgcat $) \times \mathrm{HO}($ dgcat $)$ to $\mathbb{D}$ which preserve filtered homotopy colimits in each variable is equivalent to the category of morphisms from $\operatorname{dgcat}_{\mathrm{f}}\left[\Sigma^{-1}\right] \times \operatorname{dgcat}_{\mathrm{f}}\left[\Sigma^{-1}\right]$ to $\mathbb{D}$. By induction, we prove similarly that, for any $n \geq 0$, the category of morphisms from $\mathrm{HO}(\text { dgcat })^{n}$ to $\mathbb{D}$ which preserve filtered homotopy colimits in each variable is equivalent to the category of morphisms from $\operatorname{dgcat}_{f}\left[\Sigma^{-1}\right]^{n}$ to $\mathbb{D}$. As the morphism $i$ in the above diagram (7.1.2) is symmetric monoidal, this implies that the morphism $\mathbb{R} \underline{h}$ is symmetric monoidal as well. Similarly, we see that, given any derivator $\mathbb{D}$, the category of symmetric monoidal morphisms from $\operatorname{dgcat}_{f}\left[\Sigma^{-1}\right]$ to $\mathbb{D}$ is equivalent to the category of filtered homotopy colimit preserving symmetric monoidal morphisms from $\mathrm{HO}$ (dgcat) to $\mathbb{D}$. The last assertion of this proposition thus follows from Theorem A.3 and Proposition A.9.

Let $h: \operatorname{dgcat}_{\mathrm{f}} \longrightarrow \mathrm{s} \widehat{\text { dgcat }}_{\mathrm{f}}$ be the Yoneda embedding. We denote by $P: \varnothing \longrightarrow h(\varnothing)$ the canonical map. Then, the derivator $L_{\Sigma, P}$ Hot $_{\mathrm{dgcat}_{f}}$ is simply the left Bousfield localization of $\mathrm{L}_{\Sigma} \mathrm{Hot}_{\mathrm{dgcat}_{f}}$ by $P$. Thus, it can be described as

$$
\mathrm{L}_{\Sigma, P} \operatorname{Hot}_{\mathrm{dgcat}_{\mathrm{f}}}=\mathrm{HO}\left(\mathrm{L}_{\Sigma, P} \mathrm{~s} \widehat{\operatorname{dgcat}}_{\mathrm{f}}\right)
$$

where $L_{\Sigma, P} s \widehat{\text { dgcat }}_{f}$ is the left Bousfield localization of the model category $L_{\Sigma} s \widehat{\text { dgcat }}_{f}$ by the map $P$.

Proposition 7.13. - The model category $\mathrm{L}_{\Sigma, P} \mathrm{~s} \widehat{\mathrm{dgcat}}_{\mathrm{f}}$ is symmetric monoidal, and the localization functor

$$
\mathrm{L}_{\Sigma} \mathrm{s} \widehat{\operatorname{dgcat}}_{\mathrm{f}} \longrightarrow \mathrm{L}_{\Sigma, P} \mathrm{~s} \widehat{\text { dgcat }}_{\mathrm{f}}
$$

is a symmetric monoidal left Quillen functor. In particular, the derivator $\mathrm{L}_{\Sigma, P} \mathrm{Hot}_{\mathrm{dgcat}_{\mathrm{f}}}$ is symmetric monoidal, and the localization morphism

$$
\Phi: \mathrm{L}_{\Sigma} \mathrm{Hot}_{\mathrm{dgcat}_{\mathrm{f}}} \longrightarrow \mathrm{L}_{\Sigma, P} \mathrm{Hot}_{\mathrm{dgcat}_{f}}
$$

is symmetric monoidal.

Proof. - For any dg category $\mathcal{A}$, we have $\mathcal{A} \otimes \varnothing \simeq \varnothing$. We deduce easily from this formula that condition (C) of Theorem 5.7 (with $\mathcal{M}=\mathrm{L}_{\Sigma} \mathrm{s} \widehat{\mathrm{dgcat}}_{\mathrm{f}}$ ) is satisfied, and so the proof is finished. 
Let $s \widehat{\operatorname{dgcat}}_{\mathrm{f}}$ • be the model category of pointed simplicial presheaves on $\mathrm{s} \widehat{\mathrm{dgcat}}_{\mathrm{f}}$. By virtue of Proposition 5.3, this is a symmetric monoidal model category, and the functor

$$
\mathrm{s}_{\text {dgcat }_{\mathrm{f}}} \longrightarrow \mathrm{s}_{\text {dgcat }_{\mathrm{f}} \bullet}, \quad F \longmapsto F_{+}
$$

is a symmetric monoidal left Quillen functor. We define the pointed model category $\mathrm{L}_{\Sigma, P} \mathrm{~s} \widehat{\operatorname{dgcat}}_{\mathrm{f}}, \bullet$ as the left Bousfield localization of $\mathrm{s}_{\mathrm{dgcat}_{\mathrm{f}}, \bullet}$ with respect to the set of maps $\Sigma_{+} \cup\left\{P_{+}\right\}$.

Proposition 7.14. - The model category $\mathrm{L}_{\Sigma, P} \mathrm{~s}_{\mathrm{dgcat}_{\mathrm{f}}, \bullet}$ is symmetric monoidal, and the symmetric monoidal left Quillen functor

$$
\mathrm{L}_{\Sigma, P} \mathrm{~s} \widehat{\operatorname{dgcat}}_{\mathrm{f}} \longrightarrow \mathrm{L}_{\Sigma, P} \mathrm{~s} \widehat{\operatorname{dgcat}}_{\mathrm{f}, \bullet}
$$

is a Quillen equivalence. In particular, we have a canonical equivalence of symmetric monoidal derivators

$$
\mathrm{L}_{\Sigma, P} \mathrm{Hot}_{\mathrm{dgcat}_{\mathrm{f}}} \simeq \mathrm{HO}\left(\mathrm{L}_{\Sigma, P} \mathrm{~s} \widehat{\operatorname{dgcat}}_{\mathrm{f}, \bullet}\right) .
$$

Proof. - The first assertion is a direct application of Theorem 5.7, while the second one follows from [42, Remark 8.2].

Note that the initial and terminal dg categories are Morita equivalent. This implies that the dg category 0 is sent to the point (up to weak equivalence) in $\mathrm{L}_{\Sigma, P} \mathrm{~s} \widehat{\mathrm{dgcat}}_{\mathrm{f}, \bullet}$ Let $\mathcal{E}$ be the set of morphisms of $\mathrm{L}_{\Sigma, P} \mathrm{~s} \widehat{\mathrm{dgcat}}_{\mathrm{f}, \bullet}$ of shape

$$
\text { cone }[\mathbb{R} \underline{h}(\mathcal{A}) \longrightarrow \mathbb{R} \underline{h}(\mathcal{B})] \longrightarrow \mathbb{R} \underline{h}(\mathcal{C}),
$$

associated to each exact sequence of dg categories

$$
\mathcal{A} \longrightarrow \mathcal{B} \longrightarrow \mathcal{C},
$$

with $\mathcal{B}$ in dgcat $_{\mathrm{f}}$ (where cone means homotopy cofiber). We define $\mathcal{M}$ ot $t_{\mathrm{dg}}^{\mathrm{uloc}}$ as the left Bousfield localization of $\mathrm{L}_{\Sigma, P} \mathrm{~s} \widehat{\operatorname{dgcat}}_{\mathrm{f}, \bullet}$ by $\mathcal{E}$. The derivator $\operatorname{Mot}_{\mathrm{dg}}^{\text {uloc }}$ is defined as

$$
\operatorname{Mot}_{\mathrm{dg}}^{\mathrm{uloc}}=\mathrm{HO}\left(\mathcal{M o t}_{\mathrm{dg}}^{\mathrm{uloc}}\right) \text {. }
$$

Proposition 7.15. - The model category $\mathcal{M o t}_{\mathrm{dg}}^{\mathrm{uloc}}$ is symmetric monoidal, in such a way that the left Quillen functor

$$
\mathrm{L}_{\Sigma, P} \mathrm{~s} \widehat{\operatorname{dgcat}}_{\mathrm{f}, \bullet} \longrightarrow \mathcal{M o t}_{\mathrm{dg}}^{\mathrm{uloc}}
$$

is symmetric monoidal. Under the identification of Proposition 7.13 , the induced morphism of derivators

$$
\gamma: \mathrm{L}_{\Sigma, P} \mathrm{Hot}_{\mathrm{dgcat}_{\mathrm{f}}} \longrightarrow \mathrm{Mot}_{\mathrm{dg}}^{\mathrm{uloc}}
$$

is symmetric monoidal.

Proof. - As tensoring by a $k$-flat dg category preserves exact sequences of dg categories (see [16, Proposition 1.6.3]), and as $\mathbb{R} \underline{h}$ is symmetric monoidal (see Proposition 7.12), the proof follows from Theorem 5.7. 
Finally, since by construction the model category $\mathcal{M} t_{\mathrm{dg}}^{\mathrm{uloc}}$ is symmetric monoidal and simplicially enriched, we can consider its stabilization $\mathcal{M}_{\mathrm{dg}}^{\mathrm{loc}}$, i.e. the stable model category of symmetric spectra in $\mathcal{M} t_{\mathrm{dg}}^{\text {uloc }}($ see $\S 6)$ :

$$
\mathcal{M} o t_{\mathrm{dg}}^{\mathrm{loc}}=\operatorname{Sp}^{\Sigma}\left(\mathcal{M}_{\mathrm{dg}}^{\mathrm{uloc}}\right) \text {. }
$$

The derivator $\mathrm{Mot}_{\mathrm{dg}}^{\mathrm{loc}}$ is defined as

$$
\operatorname{Mot}_{\mathrm{dg}}^{\mathrm{loc}}=\mathrm{HO}\left(\mathcal{M o t}_{\mathrm{dg}}^{\mathrm{loc}}\right) \text {. }
$$

Proposition 7.16. - The model category Mot $t_{\mathrm{dg}}^{\mathrm{loc}}$ is symmetric monoidal, and the left Quillen functor

$$
\Sigma^{\infty}: \mathcal{M o t}_{\mathrm{dg}}^{\mathrm{uloc}} \longrightarrow \mathcal{M o t}_{\mathrm{dg}}^{\mathrm{loc}}
$$

is symmetric monoidal. The induced morphism of derivators

$$
\text { stab }=\mathbb{L} \Sigma^{\infty}: \operatorname{Mot}_{\mathrm{dg}}^{\mathrm{uloc}} \longrightarrow \operatorname{Mot}_{\mathrm{dg}}^{\mathrm{loc}}
$$

is symmetric monoidal.

Proof. - This is true by construction (see Proposition A.2).

Remark 7.17. - In the construction of Mot ${ }_{\mathrm{dg}}^{\text {loc }}$ given in [42], the definition of Mot ${ }_{\mathrm{dg}}^{\text {loc }}$ was $\mathrm{HO}\left(\mathrm{Sp}^{\mathbb{N}}\left(\mathcal{M}_{\mathrm{dg}}^{\mathrm{uloc}}\right)\right)$, i.e. it used non-symmetric spectra. However, thanks to Theorem 6.1, both definitions agree up to a canonical equivalence of derivators.

Remark 7.18. - The concatenation of Propositions 7.12-7.16 show us that the localizing motivator $\mathrm{Mot}_{\mathrm{dg}}^{\mathrm{loc}}$ carries a symmetric monoidal structure $-\otimes^{\mathbb{L}}-$, making the universal localizing invariant $\mathcal{U}_{\mathrm{dg}}^{\text {loc }}$ symmetric monoidal. By Proposition A.2 the associated symmetric monoidal structure preserves homotopy colimits. Therefore, in order to conclude the proof of Theorem 7.5, it remains to show the universal property. Let $\mathbb{D}$ be a strong triangulated derivator endowed with a symmetric monoidal structure which preserves homotopy colimits in each variable. Thanks to Theorem 7.2, we have an induced equivalence of categories

$$
\left(\mathcal{U}_{\mathrm{dg}}^{\text {loc }}\right)^{*}: \underline{\operatorname{Hom}}_{!}\left(\operatorname{Mot}_{\mathrm{dg}}^{\mathrm{loc}}, \mathbb{D}\right) \stackrel{\sim}{\longrightarrow} \underline{\operatorname{Hom}}_{\mathrm{loc}}(\mathrm{HO}(\text { dgcat }), \mathbb{D}) .
$$

This implies that the induced functor

$$
\left(\mathcal{U}_{\mathrm{dg}}^{\text {loc }}\right)^{*}: \underline{\operatorname{Hom}}_{!}^{\otimes}\left(\operatorname{Mot}_{\mathrm{dg}}^{\text {loc }}, \mathbb{D}\right) \longrightarrow \underline{\operatorname{Hom}}_{\mathrm{loc}}^{\otimes}(\mathrm{HO}(\text { dgcat }), \mathbb{D}) .
$$

is faithful. More precisely, by Proposition 7.12, the category of filtered homotopy colimit preserving symmetric monoidal morphisms from $\mathrm{HO}$ (dgcat) to $\mathbb{D}$ is equivalent to the category of homotopy colimit preserving symmetric monoidal morphisms from $\mathrm{L}_{\Sigma} \mathrm{s} \widehat{\mathrm{dgcat}}_{\mathrm{f}}$ to $\mathbb{D}$. Using the universal properties of Bousfield localization and stabilization in the setting of derivators (see Theorem A.4 and Corollary A.13), we can apply Proposition A.9, and Theorem A.15 to conclude, by construction of Mot ${ }_{\mathrm{dg}}^{\mathrm{loc}}$, that (7.1.3) is an equivalence of categories. This ends the proof of Theorem 7.5. 


\section{Applications}

In this section we describe several applications of Theorem 7.5.

8.1. Non-connective $K$-theory. - Recall from Example 7.4 that non-connective algebraic $K$-theory is an example of a localizing invariant of dg categories. In [11] the authors proved that this localizing invariant becomes co-representable in the category $\operatorname{Mot}_{\mathrm{dg}}^{\mathrm{loc}}(e)$ of non-commutative motives (see Definition 7.3).

Theorem 8.1. - ([11, Theorem 7.16]) For every small $d g$ category $\mathcal{A}$, we have a natural isomorphism in the stable homotopy category of spectra

$$
\mathbb{R H o m}\left(\mathcal{U}_{\mathrm{dg}}^{\mathrm{loc}}(\underline{k}), \mathcal{U}_{\mathrm{dg}}^{\mathrm{loc}}(\mathcal{A})\right) \simeq \mathbb{K}(\mathcal{A}) .
$$

Here, $\underline{k}$ denotes the $d g$ category with one object $*$ such that $\underline{k}(*, *)=k$ in degree zero (see $\S 2.1(i))$, and $\mathbb{K}(\mathcal{A})$ the non-connective algebraic $K$-theory spectrum of $\mathcal{A}$. In particular, we obtain isomorphisms of abelian groups

$$
\operatorname{Hom}\left(\mathcal{U}_{\mathrm{dg}}^{\mathrm{loc}}(\underline{k})[n], \mathcal{U}_{\mathrm{dg}}^{\mathrm{loc}}(\mathcal{A})\right) \simeq \mathbb{K}_{n}(\mathcal{A}) \quad n \in \mathbb{Z} .
$$

A fundamental problem of the theory of non-commutatives motives is the computation of the (spectra of) morphisms between two object in the localizing motivator. Using Theorem 7.5 we give a partial solution to this fundamental problem.

Theorem 8.2. - Let $\mathcal{B}$ be a saturated dg category; see Definition 4.1. For every small $d g$ category $\mathcal{A}$, we have a natural isomorphism in the stable homotopy category of spectra

$$
\mathbb{R} \operatorname{Hom}\left(\mathcal{U}_{\mathrm{dg}}^{\mathrm{loc}}(\mathcal{B}), \mathcal{U}_{\mathrm{dg}}^{\mathrm{loc}}(\mathcal{A})\right) \simeq \mathbb{K}(\operatorname{rep}(\mathcal{B}, \mathcal{A})) .
$$

Here rep $(-,-)$ denotes the internal Hom-functor in $\mathrm{Hmo}$; see Theorem 2.16. In particular, we obtain isomorphisms of abelian groups

$$
\operatorname{Hom}\left(\mathcal{U}_{\mathrm{dg}}^{\mathrm{loc}}(\mathcal{B})[n], \mathcal{U}_{\mathrm{dg}}^{\mathrm{loc}}(\mathcal{A})\right) \simeq \mathbb{K}_{n}(\operatorname{rep}(\mathcal{B}, \mathcal{A})) \quad n \in \mathbb{Z} .
$$

Proof. - The proof is a consequence of the following weak equivalences:

$$
\begin{aligned}
\mathbb{R H o m}\left(\mathcal{U}_{\mathrm{dg}}^{\mathrm{loc}}(\mathcal{B}), \mathcal{U}_{\mathrm{dg}}^{\mathrm{loc}}(\mathcal{A})\right) & \simeq \mathbb{R} \operatorname{Hom}\left(\mathcal{U}_{\mathrm{dg}}^{\mathrm{loc}}(\underline{k}) \otimes^{\mathbb{L}} \mathcal{U}_{\mathrm{dg}}^{\mathrm{loc}}(\mathcal{B}), \mathcal{U}_{\mathrm{dg}}^{\mathrm{loc}}(\mathcal{A})\right) \\
& \simeq \mathbb{R} \operatorname{Hom}\left(\mathcal{U}_{\mathrm{dg}}^{\mathrm{lg}}(\underline{k}), \mathcal{U}_{\mathrm{dg}}^{\mathrm{loc}}(\mathcal{B})^{\vee} \otimes^{\mathbb{L}} \mathcal{U}_{\mathrm{dg}}^{\mathrm{loc}}(\mathcal{A})\right) \\
& \simeq \mathbb{R} \operatorname{Hom}\left(\mathcal{U}_{\mathrm{dg}}^{\mathrm{loc}}(\underline{k}), \mathcal{U}_{\mathrm{dg}}^{\mathrm{loc}}\left(\mathcal{B}^{\mathrm{op}} \otimes^{\mathbb{L}} \mathcal{A}\right)\right) \\
& \simeq \mathbb{R} \operatorname{Hom}\left(\mathcal{U}_{\mathrm{dg}}^{\mathrm{loc}}(\underline{k}), \mathcal{U}_{\mathrm{dg}}^{\mathrm{loc}}(\operatorname{rep}(\mathcal{B}, \mathcal{A}))\right) \\
& \simeq \mathbb{K}(\operatorname{rep}(\mathcal{B}, \mathcal{A}))
\end{aligned}
$$

Equivalence (8.1.1) follows from the fact that $\mathcal{U}_{\mathrm{dg}}^{\mathrm{loc}}(\underline{k})$ is the unit object in $\operatorname{Mot}_{\mathrm{dg}}^{\text {loc }}(e)$; see Remark 2.12 and Theorems 2.23 and 7.5. Since $\mathcal{B}$ is a saturated $\mathrm{dg}$ category, Theorem 4.8 implies that $\mathcal{B}$ is a dualizable object in $\mathrm{Hmo}$. Therefore, Equivalence 
(8.1.2) follows from the fact that $\mathcal{U}_{\mathrm{dg}}^{\mathrm{loc}}(\mathcal{B})$ is a dualizable object in $\operatorname{Mot}_{\mathrm{dg}}^{\mathrm{loc}}(e)$ (see Remark 4.7(iv) and Theorem 7.5) and from the adjunction (4.0.15) of Remark 4.7(ii) (see $\S$ A.9 for its spectral enrichment). Equivalence (8.1.3) follows from Remark 4.7(iv), from Theorem 4.8, and from the fact that the universal localizing invariant is symmetric monoidal. Equivalence (8.1.4) follows from Lemma 4.9. Finally, Equivalence (8.1.5) follows from Theorem 8.1.

Proposition 8.3. - Let $X$ and $Y$ be smooth and proper $k$-schemes. Then, we have a natural isomorphism in the stable homotopy category of spectra

$$
\mathbb{R} H o m\left(\mathcal{U}_{\mathrm{dg}}^{\text {loc }}(\operatorname{perf}(X)), \mathcal{U}_{\mathrm{dg}}^{\text {loc }}(\operatorname{perf}(Y))\right) \simeq \mathbb{K}(X \times Y) .
$$

Here, $\mathbb{K}(X \times Y)$ denotes the non-connective algebraic $K$-theory spectrum of $X \times Y$ (see $[41, \S 8])$, and perf(-) the $d g$ category constructed in Example 4.5(i). In particular, we obtain isomorphisms of abelian groups

$$
\operatorname{Hom}\left(\mathcal{U}_{\mathrm{dg}}^{\text {loc }}(\operatorname{perf}(X))[n], \mathcal{U}_{\mathrm{dg}}^{\mathrm{loc}}(\operatorname{perf}(Y))\right) \simeq \mathbb{K}_{n}(X \times Y) \quad n \in \mathbb{Z} .
$$

Proof. - Since $X$ and $Y$ are smooth and proper $k$-schemes, [51, Lemma 3.27] implies that $\operatorname{perf}(X)$ and $\operatorname{perf}(Y)$ are saturated $\mathrm{dg}$ categories. Therefore, by Theorem 8.2 we have a natural isomorphism in the stable homotopy category of spectra

$$
\mathbb{R} \operatorname{Hom}\left(\mathcal{U}_{\mathrm{dg}}^{\mathrm{loc}}(\operatorname{perf}(X)), \mathcal{U}_{\mathrm{dg}}^{\mathrm{loc}}(\operatorname{perf}(Y))\right) \simeq \mathbb{K}(\operatorname{rep}(\operatorname{perf}(X), \operatorname{perf}(Y))) .
$$

Moreover, by [48, Theorem 8.9] we have a natural isomorphism

$$
\operatorname{perf}(X \times Y) \simeq \operatorname{rep}(\operatorname{perf}(X), \operatorname{perf}(Y))
$$

in Hmo. Finally, thanks to [41, 88 Theorem 5] we have a natural isomorphism

$$
\mathbb{K}(\operatorname{perf}(X \times Y)) \simeq \mathbb{K}(X \times Y)
$$

and so the proof is finished.

Remark 8.4. - Let $Z$ be a noetherian regular scheme. Thanks to [1, Exp. I, Cor. 5.9 and Exp. II, Cor. 2.2.2.1] we have a derived Morita equivalence

$$
\operatorname{perf}(Z) \stackrel{\sim}{\longrightarrow} \mathcal{D}_{\mathrm{dg}}^{b}(\operatorname{Coh}(Z)),
$$

where the left-hand side is the saturated dg category of Example 4.5(i) and the right-hand side is the bounded derived (dg) category of coherent sheaves on $Z$. Since $\operatorname{Coh}(Z)$ is a noetherian abelian category (see $[41, \S 10.1]$ ), we conclude by [41, $\S 10.1$ Theorem 7] that

$$
\mathbb{K}_{n}(Z)=\mathbb{K}_{n}(\operatorname{perf}(Z))=0 \quad n<0 .
$$

In particular, if in Proposition 8.3 the base ring $k$ is regular and noetherian, the negative stable homotopy groups of the spectrum $\mathbb{K}(X \times Y)$ vanish. 
8.2. Kontsevich's non-commutative mixed motives. - Kontsevich introduced in $[33,34,35]$ the category of non-commutative mixed motives. His construction decomposes in three steps:

(1) First, consider the following category $\mathrm{KPM}_{k}$, enriched over symmetric spectra: the objects are the dualizable dg categories (see Definition 4.1); given saturated $\operatorname{dg}$ categories $\mathcal{A}$ and $\mathcal{B}$, the symmetric spectrum of morphisms from $\mathcal{A}$ to $\mathcal{B}$ is the non-connective $K$-theory spectrum $\mathbb{K}\left(\mathcal{A}^{\mathrm{op}} \otimes^{\mathbb{L}} \mathcal{B}\right)$; the composition corresponds to the derived tensor product of bimodules ${ }^{(2)}$.

(2) Then, take the formal triangulated envelope tri $\left(\mathrm{KPM}_{k}\right)$ of $\mathrm{KPM}_{k}$. Objects in this new category are formal finite extensions of formal shifts of objects in $\mathrm{KPM}_{k}$.

(3) Finally, add formal direct summands for projectors in tri $\left(\mathrm{KPM}_{k}\right)$. The resulting category $\mathrm{KMM}_{k}$ is what Kontsevich named the category of non-commutative mixed motives ${ }^{(3)}$.

A precise way to perform these constructions consists on seeing $\mathrm{KMM}_{k}$ as the spectral category of perfect $\mathrm{KPM}_{k}$-modules $\left(\mathrm{KMM}_{k}\right.$ is the Morita completion of $\mathrm{KPM}_{k}$; see $[46, \S 5.2]$ for a precise exposition of these constructions).

Thanks to Theorem 8.2, we are now able to construct a fully-faithful embedding of $\mathrm{KMM}_{k}$ into our category $\operatorname{Mot}_{\mathrm{dg}}^{\text {loc }}(e)$ of non-commutative motives, i.e. the base category of the localizing motivator. Note that, in contrast with Kontsevich's ad hoc definition, our category of non-commutative motives is defined purely in terms of precise universal properties.

Let $\mathcal{M} t_{\mathrm{dg}}^{\text {loc }}$ be the model category underlying the derivator Mot ${ }_{\mathrm{dg}}^{\text {loc }}$. As, by construction, $\mathcal{M} t_{\mathrm{dg}}^{\mathrm{loc}}$ is a left Bousfield localization of a category of presheaves of symmetric spectra over some small category, this model category is canonically enriched over symmetric spectra. We can thus consider the category $\operatorname{Mot}_{\mathrm{dg}}^{\text {loc }}(e)$ as a category enriched over symmetric spectra (by considering fibrant and cofibrant objects in $\mathcal{M} o t_{\mathrm{dg}}^{\mathrm{loc}}$ ).

Proposition 8.5. - There is a natural fully-faithful embedding (enriched over symmetric spectra) of Kontsevich's category of non-commutative motives $\mathrm{KMM}_{k}$ into the category $\operatorname{Mot}_{\mathrm{dg}}^{\mathrm{loc}}(e)$. The essential image is the thick triangulated subcategory spanned by motives of saturated dg categories.

Proof. - Given saturated dg categories $\mathcal{A}$ and $\mathcal{B}$, Lemma 4.9 implies that we have a natural isomorphism in $\mathrm{Hmo}$

$$
\mathcal{A}^{\mathrm{op}} \otimes^{\mathbb{L}} \mathcal{B} \simeq \operatorname{rep}(\mathcal{A}, \mathcal{B})
$$

\footnotetext{
${ }^{(2)}$ This category is the non-commutative (and derived) analogue of Grothendieck's category of pure motives: PM stands for Pure Motives, while K stands for both Kontsevich and $K$-theory.

${ }^{(3)} \mathrm{MM}$ stands for Mixed Motives, while K stands for both Kontsevich and $K$-theory.
} 
Therefore, using Theorem 8.2 we obtain a natural fully-faithful spectral functor

$$
\mathrm{KPM}_{k} \longrightarrow \operatorname{Mot}_{\mathrm{dg}}^{\mathrm{loc}}(e) \quad \mathcal{A} \mapsto \mathcal{U}_{\mathrm{dg}}^{\mathrm{loc}}(\mathcal{A})
$$

By construction of $\mathrm{KMM}_{k}$, [46, Proposition 5.3.1] implies that this functor extends (uniquely) to a spectral functor

$$
\mathrm{KMM}_{k} \longrightarrow \operatorname{Mot}_{\mathrm{dg}}^{\mathrm{loc}}(e) \text {. }
$$

In order to show that this functor is (homotopically) fully-faithful, it is sufficient to prove that its restriction to a generating family of $\mathrm{KMM}_{k}$ is fully faithful, which holds by construction.

8.3. Chern characters. - In [11] the authors used the co-representability Theorem 8.1 to classify all natural transformations out of non-connective $K$-theory. More precisely, they proved in [11, Theorem 8.1] that given a localizing invariant $L$, with values in the derivators of spectra, the data of a natural transformation $\mathbb{K}(-) \Rightarrow L(-)$ is equivalent to the datum of a single class in the stable homotopy group $\pi_{0} L(\underline{k})$. From this result they obtained higher Chern characters (resp. higher trace maps), from non-connective $K$-theory to (topological) cyclic homology (resp. to (topological) Hochschild homology); see [11, Theorem 8.4].

However, negative cyclic homology $H^{-}$and periodic cyclic homology $H P$ do not preserve filtered homotopy colimits since they are defined using infinite products; see $[38, \S 5.1]$. Therefore, they are not examples of localizing invariants and so the theory developed in [11] is not directly applicable in these cases. Nevertheless, we shall explain below why and how negative cyclic homology and periodic cyclic homology fit naturally in our framework; see Examples 8.10 and 8.11.

Let $\mathbb{D}$ be a strong triangulated derivator endowed with a symmetric monoidal structure (with unit 1) which preserves homotopy colimits in each variable (see §A.5), and

$$
E: \mathrm{HO}(\text { dgcat }) \longrightarrow \mathbb{D}
$$

a symmetric monoidal localizing invariant (see Definition 7.6). Thanks to Theorem 7.5 there is a (unique) symmetric monoidal homotopy colimit preserving morphism of derivators $E_{\mathrm{gm}}$ which makes the diagram

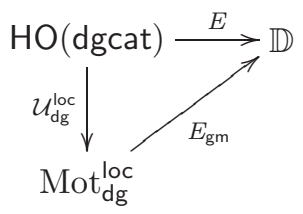

commute (up to unique 2-isomorphism). 
Definition 8.6. - The morphism $E_{\mathrm{gm}}$ is called the geometric realization of $E$. Since by hypothesis $\mathbb{D}$ is triangulated, we have a natural morphism of derivators

$$
\mathbb{R} \operatorname{Hom}(\mathbf{1},-): \mathbb{D} \longrightarrow \mathrm{HO}\left(\mathrm{Sp}^{\mathbb{N}}\right) \quad(\text { see } \S \mathrm{A} .9) \text {. }
$$

The composed morphism

$$
E_{\mathrm{abs}}:=\mathbb{R} \operatorname{Hom}\left(1, E_{\mathrm{gm}}(-)\right): \operatorname{Mot}_{\mathrm{dg}}^{\mathrm{loc}} \longrightarrow \mathrm{HO}\left(\mathrm{Sp}^{\mathbb{N}}\right)
$$

is called the absolute realization of $E$.

Given a symmetric monoidal localizing invariant $E$, we have two objects associated to a non-commutative motive $M \in \operatorname{Mot}_{\mathrm{dg}}^{\text {loc }}(e)$ : its geometric realization $E_{\mathrm{gm}}(M)$ and its absolute realization $E_{\text {abs }}(M)$. Although the morphism $E_{\mathrm{gm}}$ always preserves homotopy colimits, this is not always the case for the morphism $E_{\mathrm{abs}}$; a sufficient (and almost necessary) condition for $E_{\text {abs }}$ to preserve homotopy colimits is that the unit $\mathbf{1}$ of $\mathbb{D}$ is a compact object.

Proposition 8.7. - The geometric realization of $E$ induces a canonical Chern character

$$
\mathbb{I K}(-) \Rightarrow \mathbb{R} \operatorname{Hom}(\mathbf{1}, E(-)) \simeq E_{\mathrm{abs}}\left(\mathcal{U}_{\mathrm{dg}}^{\text {loc }}(-)\right) .
$$

Here, $\mathbb{K}(-)$ and $E_{\mathrm{abs}}\left(\mathcal{U}_{\mathrm{dg}}^{\mathrm{loc}}(-)\right)$ are two morphisms of derivators defined on $\mathrm{HO}$ (dgcat).

Proof. - The geometric realization of $E$ is symmetric monoidal and so it sends the unit object $\mathcal{U}_{\mathrm{dg}}^{\text {loc }}(\underline{k})$ to $\mathbf{1} \in \mathbb{D}$. Therefore, given a small $\operatorname{dg}$ category $\mathcal{A}$, we obtain an induced map

$\mathbb{K}(\mathcal{A}) \simeq \mathbb{R} \operatorname{Hom}_{\mathrm{Mot}_{\mathrm{dg}}^{\mathrm{loc}}}\left(\mathcal{U}_{\mathrm{dg}}^{\mathrm{loc}}(\underline{k}), \mathcal{U}_{\mathrm{dg}}^{\mathrm{loc}}(\mathcal{A})\right) \longrightarrow \mathbb{R} \operatorname{Hom}_{\mathbb{D}}\left(\mathbf{1}, E_{\mathrm{gm}}\left(\mathcal{U}_{\mathrm{dg}}^{\mathrm{loc}}(\mathcal{A})\right)\right)=E_{\mathrm{abs}}\left(\mathcal{U}_{\mathrm{dg}}^{\text {loc }}(\mathcal{A})\right)$, where the left-hand side equivalence follows from Theorem 8.1. Since this induced map is functorial in $\mathcal{A}$, the proof is finished.

Let us now give some examples which illustrate Proposition 8.7.

Example 8.8 (Non-connective $K$-theory). - The tautological version of the situation above is: for $E=\mathcal{U}_{\mathrm{dg}}^{\text {loc }}, E_{\mathrm{gm}}$ is by definition the identity of Mot $_{\mathrm{dg}}^{\text {loc }}$ (see Theorem 7.5), while $E_{\text {abs }}=\mathbb{K}$ is non-connective $K$-theory (see Theorem 8.1). The corresponding Chern character is the identity, and this is in this precise sense that non-connective $K$-theory is initial among absolute homology theories.

Example 8.9 (Hochschild homology). — Take for $E$ the symmetric monoidal localizing invariant

$$
H H: \mathrm{HO}(\text { dgcat }) \longrightarrow \mathrm{HO}(\mathcal{C}(k))
$$

of Example 7.9. In this case, there is no difference (up to the Dold-Kan correspondance relating complexes of $k$-modules and spectra) between the geometric and the absolute realization: if we consider $\mathrm{HO}(\mathcal{C}(k))$ as enriched over itself, then the morphism

$$
\mathbb{R} \operatorname{Hom}(k,-): \mathrm{HO}(\mathcal{C}(k)) \longrightarrow \mathrm{HO}(\mathcal{C}(k))
$$


is (isomorphic to) the identity. Therefore by Proposition 8.7, we obtain a canonical Chern character

$$
\mathbb{K}(-) \Rightarrow H H(-) \text {. }
$$

Example 8.10 (Negative cyclic homology). - Take for $E$ the symmetric monoidal localizing invariant

$$
C: \mathrm{HO}(\text { dgcat }) \longrightarrow \mathrm{HO}(\mathcal{C}(\Lambda))
$$

of Example 7.10. Given a small dg category $\mathcal{A}$, we have an equivalence

$$
C_{\mathrm{abs}}\left(\mathcal{U}_{\mathrm{dg}}^{\mathrm{loc}}(\mathcal{A})\right)=\mathbb{R} \operatorname{Hom}(k, C(\mathcal{A})) \simeq H C^{-}(\mathcal{A}),
$$

where $H C^{-}(\mathcal{A})$ denotes the negative cyclic homology complex of $\mathcal{A}$; see [32, §2.2]. Therefore by Proposition 8.7, we obtain a canonical Chern character

$$
\mathbb{K}(-) \Rightarrow H C^{-}(-) .
$$

Example 8.11 (Periodic cyclic homology). - Take for $E$ the symmetric monoidal localizing invariant

$$
(P \circ C): \mathrm{HO}(\text { dgcat }) \longrightarrow \mathrm{HO}(k[u] \text {-Comod })
$$

of Example 7.11. Assuming that the ground ring $k$ is a field, for any small dg category $\mathcal{A}$, we have a natural identification

$$
(P \circ C)_{\mathrm{abs}}\left(\mathcal{U}_{\mathrm{dg}}^{\mathrm{loc}}(\mathcal{A})\right)=\mathbb{R} \operatorname{Hom}(k[u],(P \circ C)(\mathcal{A})) \simeq H P(\mathcal{A}),
$$

where $H P(\mathcal{A})$ denotes the periodic cyclic homology complex of $\mathcal{A}$. This can be seen as follows. Given a mixed complex $M$ (see Example 7.10), a map $k[u] \rightarrow P(M)$ in $k[u]$-Comod corresponds to a collection of maps $k \rightarrow\left(M \otimes_{\Lambda}^{\mathbb{L}} k\right)[2 n], n \geq 0$, in $\mathcal{C}(k)$ which are compatible with the operator $S$. In other words, these data correspond to a map in $\mathcal{C}(k)$ from $k$ to the tower

$\cdots \stackrel{S}{\longrightarrow}\left(M \otimes_{\Lambda}^{\mathbb{L}} k\right)[-2 n] \stackrel{S}{\longrightarrow}\left(M \otimes_{\Lambda}^{\mathbb{L}} k\right)[-2 n+2] \stackrel{S}{\longrightarrow} \cdots \stackrel{S}{\longrightarrow}\left(M \otimes_{\Lambda}^{\mathbb{L}} k\right)[-2] \stackrel{S}{\longrightarrow} M \otimes_{\Lambda}^{\mathbb{L}} k$.

In other words, we have:

$$
\mathbb{R} \operatorname{Hom}(k[u], P(M)) \simeq \operatorname{holim}_{n}\left(M \otimes_{\Lambda}^{\mathbb{L}} k\right)[-2 n] .
$$

The Milnor short exact sequence [24, Proposition 7.3.2] applied to this homotopy limit corresponds to the short exact sequence

$0 \longrightarrow 亡_{n}^{\lim _{n}^{1}} H_{i+2 n-1}\left(M \otimes_{\Lambda}^{\mathbb{L}} k\right) \longrightarrow \operatorname{Hom}(k[u], P(M)[-i]) \longrightarrow{\underset{n}{\lim }}_{H_{i+2 n}}\left(M \otimes_{\Lambda}^{\mathbb{L}} k\right) \longrightarrow 0$.

Now, let $\mathcal{A}$ be a small dg category. The above arguments, with $M=C(\mathcal{A})$, allow us to deduce the formula

$$
\mathbb{R H o m}(k[u],(P \circ C)(\mathcal{A})) \simeq \operatorname{holim}_{n}\left(C(\mathcal{A}) \otimes_{\Lambda}^{\mathbb{L}} k\right)[-2 n] \simeq H P(\mathcal{A}) .
$$

Therefore, by Proposition 8.7, we obtain a canonical Chern character

$$
\mathbb{K}(-) \Rightarrow H P(-) \text {. }
$$


8.4. Toën's secondary $K$-theory. — Toën introduced in $[49,50]$ a "categorified" version of algebraic $K$-theory named secondary $K$-theory; see [52] for a survey article.

Definition 8.12 ([49, §5.4]). - Given a commutative $\operatorname{ring} k$, let $\mathbb{Z}\left[\mathrm{Hmo}_{\mathrm{sat}, k}\right]$ be the free abelian group on the isomorphism classes of objects in $\mathrm{Hmo}_{\text {sat }, k}$ (see Notation 4.3). The secondary $K$-theory group $K_{0}^{(2)}(k)$ of $k$ is the quotient of $\mathbb{Z}\left[\mathrm{Hmo}_{\mathrm{sat}, k}\right]$ by the relations $[\mathcal{B}]=[\mathcal{A}]+[\mathcal{C}]$ associated to exact sequences (see Definition 7.1)

$$
\mathcal{A} \longrightarrow \mathcal{B} \longrightarrow \mathcal{C}
$$

of saturated dg categories.

Remark 8.13. - (i) Thanks to Theorem 4.8 the category $\mathrm{Hmo}_{\text {sat }, k}$ coincides with the category of dualizable objects in $\mathrm{Hmo}_{k}$. Therefore, by Remark 4.7(iii), the derived tensor product in $\mathrm{Hmo}_{k}$ restricts to a bifunctor

$$
-\otimes^{\mathbb{L}}-: \mathrm{Hmo}_{\mathrm{sat}, k} \times \mathrm{Hmo}_{\mathrm{sat}, k} \longrightarrow \mathrm{Hmo}_{\mathrm{sat}, k} .
$$

By [16, Proposition 1.6.3] the derived tensor product preserves exact sequences (in both variables), and so we obtain a commutative ring structure on $K_{0}^{(2)}(k)$.

(ii) Given a ring homomorphism $k \rightarrow k^{\prime}$, we have a derived base change functor

$$
-\otimes_{k}^{\mathbb{L}} k^{\prime}: \mathrm{Hmo}_{k} \longrightarrow \mathrm{Hmo}_{k^{\prime}} \quad \mathcal{A} \mapsto \mathcal{A} \otimes_{k}^{\mathbb{L}} k^{\prime} .
$$

This functor preserves exact sequences and is symmetric monoidal. Therefore, by Theorem 4.8 and Remark 4.7(iv), we obtain a ring homomorphism

$$
K_{0}^{(2)}(k) \longrightarrow K_{0}^{(2)}\left(k^{\prime}\right) \text {. }
$$

In conclusion, secondary $K$-theory is a functor $K_{0}^{(2)}(-)$ from the category of commutative rings to itself.

One of the motivations for the study of this secondary $K$-theory was its expected connection with an hypothetical Grothendieck ring of motives in the non-commutative setting; see [50, page 1]. Thanks to Theorem 7.5, we are now able to make this connection precise; see Remarks 8.17-8.19. We shall use the following well known property of dualizable objects in a triangulated category.

Proposition 8.14. - Let $\mathcal{C}$ be a closed symmetric monoidal triangulated category. Then, the category $\mathcal{C}^{\vee}$ of dualizable objects in $\mathcal{C}$ (see Definition 4.6) is a symmetric monoidal thick triangulated subcategory of $\mathcal{C}$.

Proof. - The fact that dualizable objects are stable under tensor product is clear; see Remark 4.7(iii). For an object $X$ in $\mathcal{C}$, set $X^{\vee}=\underline{\operatorname{Hom}}(X, \mathbf{1})$. Given two objects $X$ and $Y$ in $\mathcal{C}$, we have a canonical map

$$
u_{X, Y}: X^{\vee} \otimes Y \longrightarrow \underline{\operatorname{Hom}}(X, Y)
$$

which corresponds by adjunction to the map $X^{\vee} \otimes X \otimes Y \rightarrow Y$ obtained by tensoring $Y$ with the evaluation map $X \otimes X^{\vee} \rightarrow \mathbf{1}$. The object $X$ is dualizable if and only if 
the map $u_{X, Y}$ is invertible for any object $Y$. Since for any fixed object $Y$ the map $u_{X, Y}$ is a natural transformation of triangulated functors, we conclude that $\mathcal{C}^{\vee}$ is a thick triangulated subcategory of $\mathcal{C}$.

Notation 8.15. - Thanks to Theorem 7.5 the localizing motivator carries a symmetric monoidal structure, and so its base category $\operatorname{Mot}_{\mathrm{dg}, k}^{\text {loc }}(e)$ is a symmetric monoidal triangulated category. Therefore by Proposition 8.14, the category $\operatorname{Mot}_{\mathrm{dg}, k}^{\mathrm{loc}}(e)^{\vee}$ of dualizable objects is a symmetric monoidal thick triangulated subcategory of $\operatorname{Mot}_{\mathrm{dg}, k}^{\mathrm{loc}}(e)$.

Let $\mathrm{KMM}_{k}$ be Kontsevich's category of non-commutative mixed motives. Thanks to Proposition 8.5, we can identify it with the thick triangulated subcategory of $\operatorname{Mot}_{\mathrm{dg}, k}^{\mathrm{loc}}(e)^{\vee}$ generated by objects of shape $\mathcal{U}_{\mathrm{dg}}^{\mathrm{loc}}(\mathcal{A})$, where $\mathcal{A}$ runs over the family of saturated $\mathrm{dg}$ categories over $k$. Therefore, $\mathrm{KMM}_{k}$ is naturally a rigid symmetric monoidal triangulated category.

Definition 8.16. - Let $k$ be a commutative ring. The Grothendieck ring $\mathcal{K}_{0}(k)$ of non-commutative motives over $k$ is the Grothendieck ring $K_{0}\left(\mathrm{KMM}_{k}\right)$.

Remark 8.17 (Non-triviality). - Recall from Example 7.9 the construction of the symmetric monoidal localizing invariant

$$
H H: \mathrm{HO}(\text { dgcat }) \longrightarrow \mathrm{HO}(\mathcal{C}(k)) \text {. }
$$

By restricting its geometric realization (see Definition 8.6) to the base category, we obtain a symmetric monoidal triangulated functor

$$
H H_{\mathrm{gm}}(e): \operatorname{Mot}_{\mathrm{dg}, k}^{\mathrm{loc}}(e) \longrightarrow \mathrm{HO}(\mathcal{C}(k))(e)=\mathcal{D}(k) .
$$

Recall that the dualizable objects in $\mathcal{D}(k)$ are precisely the perfect complexes of $k$ modules. Therefore, by Remark 4.7 (iv), $H_{\mathrm{gm}}(e)$ sends dualizable objects to perfect complexes and so it induces a ring homomorphism

$$
r \mathcal{K}_{0}: \mathcal{K}_{0}(k)=K_{0}\left(\mathrm{KMM}_{k}\right) \longrightarrow K_{0}\left(\mathcal{D}_{c}(k)\right)=K_{0}(k) .
$$

Finally, since $K_{0}(k)$ is non-trivial we conclude that $\mathcal{K}_{0}(k)$ is also non-trivial.

Remark 8.18 (Functoriality). - Given a ring homomorphism $k \rightarrow k^{\prime}$, we have a base change functor

$$
-\otimes_{k} k^{\prime}: \operatorname{dgcat}_{k} \longrightarrow \operatorname{dgcat}_{k^{\prime}} \quad \mathcal{A} \mapsto \mathcal{A} \otimes_{k} k^{\prime} .
$$

This functor gives rise to a morphism of derivators

$$
-\otimes_{k}^{\mathbb{L}} k^{\prime}: \mathrm{HO}\left(\text { dgcat }_{k}\right) \longrightarrow \mathrm{HO}\left(\text { dgcat }_{k^{\prime}}\right),
$$

which is symmetric monoidal, preserves homotopy colimits (and the point), and satisfies localization; see Theorem 7.2. Therefore, the composition

$$
\mathrm{HO}\left(\text { dgcat }_{k}\right) \stackrel{-\otimes_{k}^{\mathbb{L}} k^{\prime}}{\longrightarrow} \mathrm{HO}\left(\text { dgcat }_{k^{\prime}}\right) \stackrel{\mathcal{U}_{\mathrm{dg}}^{\mathrm{loc}}}{\longrightarrow} \mathrm{Mot}_{\mathrm{dg}, k^{\prime}}^{\mathrm{loc}}
$$


is a symmetric monoidal localizing invariant; see 7.6. Using Theorem 7.5, we obtain a (unique) symmetric monoidal morphism, which we still denoted by $-\otimes_{k}^{\mathbb{L}} k^{\prime}$, making the diagram

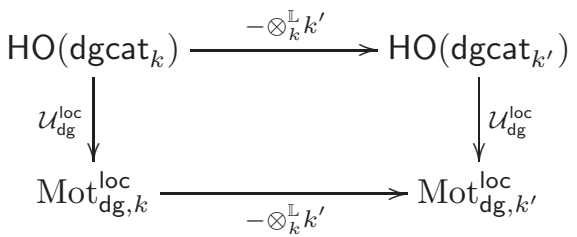

commute (up to 2-isomorphism). By restricting ourselves to the base categories, we have a symmetric monoidal triangulated functor

$$
-\otimes_{k}^{\mathbb{L}} k^{\prime}: \operatorname{Mot}_{\mathrm{dg}, k}^{\mathrm{loc}}(e) \longrightarrow \operatorname{Mot}_{\mathrm{dg}, k^{\prime}}^{\mathrm{loc}}(e) .
$$

As the (derived) change of scalars functor preserves saturated dg categories, we obtain then an induced ring homomorphism

$$
\mathcal{K}_{0}(k) \longrightarrow \mathcal{K}_{0}\left(k^{\prime}\right)
$$

In conclusion, the Grothendieck ring of non-commutative motives is a functor $\mathcal{K}_{0}(-)$ from the category of commutative rings to itself.

Remark 8.19 (Connection). — Thanks to Theorem 7.5, the functor

$$
\mathcal{U}_{\mathrm{dg}}^{\mathrm{loc}}: \mathrm{Hmo}_{k} \longrightarrow \operatorname{Mot}_{\mathrm{dg}, k}^{\mathrm{loc}}(e)
$$

is symmetric monoidal. By construction it sends exact sequences to distinguished triangles, and so it induces a ring homomorphism

$$
\Phi(k): K_{0}^{(2)}(k) \longrightarrow \mathcal{K}_{0}(k) .
$$

Note that this ring homomorphism is not necessarily surjective because of step (3) in the construction of $\mathrm{KMM}_{k}$. However, the image of $\Phi(k)$ can be described as the Grothendieck group of the triangulated category tri $\left(\mathrm{KPM}_{k}\right)$ : by cofinality the Grothendieck ring $K_{0}\left(\operatorname{tri}\left(\mathrm{KPM}_{k}\right)\right)$ is a subring of $\mathcal{K}_{0}(k)$ and by dévissage $\Phi(k)$ surjects on $K_{0}\left(\operatorname{tri}\left(\mathrm{KPM}_{k}\right)\right)$. Moreover, the above (up to 2-isomorphism) commutative square (8.4.2) shows us that the ring homomorphism (8.4.3) gives rise to a natural transformation of functors

$$
K_{0}^{(2)}(-) \Rightarrow \mathcal{K}_{0}(-), \quad k \mapsto \Phi(k) .
$$

Now, let $R$ be a commutative ring and $l: K_{0}^{(2)}(k) \rightarrow R$ a realization of $K_{0}^{(2)}(k)$, i.e. a ring homomorphism. Note that if there exists a symmetric monoidal localizing invariant

$$
\mathrm{HO}\left(\text { dgcat }_{k}\right) \longrightarrow \mathbb{D}
$$

whose induced ring homomorphism (see Proposition 8.14)

$$
K_{0}^{(2)}(k) \longrightarrow K_{0}\left(\mathbb{D}(e)^{\vee}\right)
$$


identifies with $l$, then $l$ factors through $\Phi(k)$. An interesting example is proved by Toën's rank map (see $[49, \S 5.4]$ )

$$
r k_{0}: K_{0}^{(2)} \longrightarrow K_{0}(k)
$$

Thanks to [49, §5.4 Lemma 3] this rank map is induced from the symmetric monoidal localizing invariant

$$
H H: \mathrm{HO}\left(\text { dgcat }_{k}\right) \longrightarrow \mathrm{HO}(\mathcal{C}(k))
$$

of Example 7.9. Therefore, it corresponds to the following composition

$$
K_{0}^{(2)}(k) \stackrel{\Phi(k)}{\longrightarrow} \mathcal{K}_{0}(k) \stackrel{r \mathcal{K}_{Q}}{\longrightarrow} K_{0}(k),
$$

where $r \mathcal{K}_{0}$ is the ring homomorphism (8.4.1) of Remark 8.17.

\subsection{Euler characteristic. -}

Definition 8.20. - Let $\mathcal{C}$ be a symmetric monoidal category with monoidal product $\otimes$ and unit object 1 . Given a dualizable object $X$ in $\mathcal{C}$ (see Definition 4.6) its Euler characteristic $\chi(X)$ is the following composition

$$
\chi(X): \mathbf{1} \stackrel{\delta}{\longrightarrow} X^{\vee} \otimes X \stackrel{\tau}{\longrightarrow} X \otimes X^{\vee} \stackrel{\text { ev }}{\longrightarrow} \mathbf{1},
$$

where $\tau$ denotes the symmetry isomorphism.

Remark 8.21. - Let $\mathcal{C}$ is a well behaved symmetric monoidal triangulated category; e.g. $\mathcal{C}=\mathbb{D}(e)$ for some symmetric monoidal triangulated derivator $\mathbb{D}$. Then, thanks to [37, Theorem 1.9], the Euler characteristic gives rise to a ring homomorphism

$$
\chi: K_{0}\left(\mathcal{C}^{\vee}\right) \longrightarrow \operatorname{Hom}_{\mathcal{C}}(\mathbf{1}, \mathbf{1}) .
$$

Proposition 8.22. - Let $\mathcal{A}$ be a saturated dg category. Then its Euler characteristic $\chi(\mathcal{A})$ in $\mathrm{Hmo}$ is the isomorphism class of $\mathcal{D}_{c}(k)$ which is associated to the (perfect) Hochschild homology complex $H H(\mathcal{A})$ of $\mathcal{A}$ (see Example 7.9).

Proof. - From Theorem 4.8 (and its proof) we see that the dual of $\mathcal{A}$ is its opposite $\operatorname{dg}$ category $\mathcal{A}^{\mathrm{op}}$, and that the following composition in $\mathrm{Hmo}$

$$
\chi(\mathcal{A}): \underline{k} \stackrel{[\mathcal{A}(-,-)]}{\longrightarrow} \mathcal{A}^{\mathrm{op}} \otimes^{\mathbb{L}} \mathcal{A} \stackrel{\tau}{\longrightarrow} \mathcal{A} \otimes \mathbb{L} \mathcal{A}^{\mathrm{op}} \stackrel{[\mathcal{A}(-,-)]}{\longrightarrow} \underline{k}
$$

corresponds to the complex

$$
\mathcal{A}(-,-) \bigotimes_{\mathcal{A}^{\mathrm{op} \otimes \mathbb{L}} \mathcal{A}}^{\mathbb{L}} \mathcal{A}(-,-) .
$$

By [38, Proposition 1.1.13] this complex of $k$-modules computes Hochschild homology of $\mathcal{A}$ (with coefficients in itself), which achieves the proof. 
Proposition 8.23. - Let $F: \mathcal{C} \rightarrow \mathcal{C}^{\prime}$ be a symmetric monoidal functor between symmetric monoidal categories with unit objects $\mathbf{1}$ and $\mathbf{1}^{\prime}$. Then, given a dualizable object $X$ in $\mathcal{C}$, the Euler characteristic $\chi(F(X))$ of $F(X)$ agrees with $F(\chi(X))$ on $\mathbf{1}^{\prime} \simeq F(\mathbf{1})$

Proof. - It is a straightforward consequence of the definitions. The details are left as an exercise for the reader.

Proposition 8.24. - Let $\mathcal{A}$ be a saturated dg category. Then, $\chi\left(\mathcal{U}_{\mathrm{dg}}^{\mathrm{loc}}(\mathcal{A})\right)$ is the element of the Grothendieck group $K_{0}(k)$ which is associated to the (perfect) Hochschild homology complex $H H(\mathcal{A})$ of $\mathcal{A}$.

Proof. - Thanks to Theorem 7.5, the universal localizing invariant $\mathcal{U}_{\mathrm{dg}}^{\mathrm{loc}}$ is symmetric monoidal. Using Theorem 8.1, we see that the map

$$
\mathcal{U}_{\mathrm{dg}}^{\mathrm{loc}}(e): \operatorname{Iso}_{\mathcal{D}}(k) \simeq \operatorname{Hom}_{\mathrm{Hmo}}(\underline{k}, \underline{k}) \longrightarrow \operatorname{Hom}\left(\mathcal{U}_{\mathrm{dg}}^{\mathrm{loc}}(\underline{k}), \mathcal{U}_{\mathrm{dg}}^{\mathrm{loc}}(\underline{k})\right) \simeq K_{0}(k)
$$

sends an element in Iso $\mathcal{D}_{c}(k)$ to the corresponding class in the Grothendieck group $K_{0}\left(\mathcal{D}_{c}(k)\right) \simeq K_{0}(k)$. Hence, Theorem 4.8 and Proposition 8.23 achieve the proof.

Example 8.25. - Recall from Example 4.5 that, given a smooth and proper $k$ scheme $X$, we have a saturated dg category $\operatorname{perf}(X)$ which enhances the category of compact objects in $\mathcal{D}_{q c o h}(X)$. Thanks to Keller $[31,32]$ the Hochschild homology of $\operatorname{perf}(X)$ (see Example 7.9) agrees with the Hochschild homology of $X$ in the sense of Weibel [54]. Therefore, by Proposition 8.24 the Euler characteristic of $\mathcal{U}_{\mathrm{dg}}^{\text {loc }}(\operatorname{perf}(X))$ is the element of the Grothendieck group $K_{0}(k)$ which is associated to the (perfect) Hochschild homology complex $H H(X)$ of $X$.

When $k$ is the field of complex numbers, the Grothendieck ring $K_{0}(\mathbb{C})$ is naturally isomorphic to $\mathbb{Z}$ and the Hochschild homology of $X$ agrees with the Hodge cohomology $H^{*}\left(X, \Omega_{X}^{*}\right)$ of $X$. Therefore, when we work over $\mathbb{C}$, the Euler characteristic of $\mathcal{U}_{\mathrm{dg}}^{\text {loc }}(\operatorname{perf}(X))$ is the classical Euler characteristic of $X$.

A

\section{Grothendieck derivators}

The original reference for the theory of derivators is Grothendieck's manuscript [28] and Heller's monograph [21]. See also [7, 9, 12, 42].

A.1. Prederivators. - A prederivator $\mathbb{D}$ consists of a strict contravariant 2functor from the 2-category of small categories to the 2-category of categories

$$
\mathbb{D}: \mathrm{Cat}^{\mathrm{op}} \longrightarrow \mathrm{CAT} \text {. }
$$

Prederivators organize themselves naturally in a 2-category: the 1-morphisms (usually called morphisms) are the pseudo natural transformations and the 2-morphisms are 
the modifications; see $[12, \S 5]$ for details. Given prederivators $\mathbb{D}$ and $\mathbb{D}^{\prime}$, we denote by $\underline{\operatorname{Hom}}\left(\mathbb{D}, \mathbb{D}^{\prime}\right)$ the category of morphisms.

Given a category $\mathcal{M}$, we denote by $\underline{\mathcal{M}}$ the prederivator defined for every small category $X$ by

$$
\underline{\mathcal{M}}(X):=\operatorname{Fun}\left(X^{\mathrm{op}}, \mathcal{M}\right),
$$

where $\operatorname{Fun}\left(X^{\mathrm{op}}, \mathcal{M}\right)$ is the category of presheaves on $X$ with values in $\mathcal{M}$. If $\mathcal{W}$ is a class of morphisms in $\mathcal{M}$, we denote by $\underline{\mathcal{M}}\left[\mathcal{W}^{-1}\right]$ the prederivator defined for every small category $X$ by

$$
\underline{\mathcal{M}}\left[\mathcal{W}^{-1}\right](X):=\operatorname{Fun}\left(X^{\mathrm{op}}, \mathcal{M}\right)\left[\mathcal{W}^{-1}\right] .
$$

Here $\operatorname{Fun}\left(X^{\mathrm{op}}, \mathcal{M}\right)\left[\mathcal{W}^{-1}\right]$ is the localization of Fun $\left(X^{\mathrm{op}}, \mathcal{M}\right)$ with respect to the class of morphism which belong termwise to $\mathcal{W}$. Note that the assignment $(\mathcal{M}, \mathcal{W}) \mapsto$ $\underline{\mathcal{M}}\left[\mathcal{W}^{-1}\right]$ is 2 -functorial, i.e. given a natural transformation

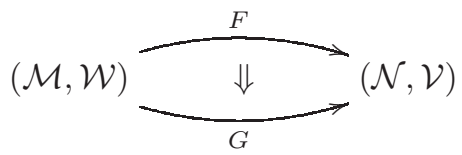

between functors, such that $F(\mathcal{W}) \subset(\mathcal{V})$ and $G(\mathcal{W}) \subset(\mathcal{V})$, we obtain an induced 2-morphism

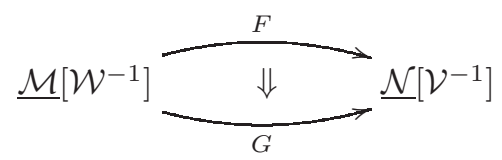

of prederivators.

A.2. Derivators. - A derivator is a prederivator which is subject to certain conditions, the main ones being that for any functor $u: X \rightarrow Y$ between small categories, the inverse image functor

$$
u^{*}=\mathbb{D}(u): \mathbb{D}(Y) \longrightarrow \mathbb{D}(X)
$$

has a left adjoint, called the homological direct image functor,

$$
u_{!}: \mathbb{D}(X) \longrightarrow \mathbb{D}(Y),
$$

as well as right adjoint, called the cohomological direct image functor

$$
u_{*}: \mathbb{D}(X) \longrightarrow \mathbb{D}(Y) \text {. }
$$

See [7] for details. Similarly to the case of prederivators, derivators organize themselves in a 2-category. Given derivators $\mathbb{D}$ and $\mathbb{D}^{\prime}$, we denote by $\underline{\text { Hom }}_{\mathrm{flt}}\left(\mathbb{D}, \mathbb{D}^{\prime}\right)$ the category of morphisms of derivators which preserve filtered homotopy colimits, and by $\underline{\mathrm{Hom}}_{!}\left(\mathbb{D}, \mathbb{D}^{\prime}\right)$ the category of morphisms of derivators which commute with all homotopy colimits; see [7, 9]. 
The essential example of a derivator to keep in mind is the derivator $\mathbb{D}=\mathrm{HO}(\mathcal{M})$ associated to a (complete and cocomplete) Quillen model category $\mathcal{M}$ (see [7, Theorem 6.11]), which is defined for every small category $X$ by

$$
\operatorname{HO}(\mathcal{M})(X):=\operatorname{Ho}\left(\operatorname{Fun}\left(X^{\mathrm{op}}, \mathcal{M}\right)\right) .
$$

In this case, any colimit (resp. limit) preserving left (resp. right) Quillen functor induces a morphism of derivators which preserves homotopy colimits (resp. limits); see [7, Proposition 6.12].

Finally, we denote by $e$ the 1-point category with one object and one (identity) morphism. Heuristically, the category $\mathbb{D}(e)$ is the basic "derived" category under consideration in the derivator $\mathbb{D}$. For instance, if $\mathbb{D}=\mathrm{HO}(\mathcal{M})$ then $\mathbb{D}(e)=\mathrm{Ho}(\mathcal{M})$ is the usual homotopy category of $\mathcal{M}$.

\section{A.3. Properties. -}

(i) A derivator $\mathbb{D}$ is called strong if for every finite free category $X$ and every small category $Y$, the natural functor $\mathbb{D}(X \times Y) \rightarrow \operatorname{Fun}\left(X^{\mathrm{op}}, \mathbb{D}(Y)\right)$ is full and essentially surjective.

(ii) A derivator $\mathbb{D}$ is called regular if sequential homotopy colimits commute with finite products and homotopy pullbacks.

(iii) A derivator $\mathbb{D}$ is called pointed if for any closed immersion $i: Z \rightarrow X$ in Cat the cohomological direct image functor $i_{*}: \mathbb{D}(Z) \rightarrow \mathbb{D}(X)$ has a right adjoint, and if, dually, for any open immersion $j: U \rightarrow X$ the homological direct image functor $j !: \mathbb{D}(U) \rightarrow \mathbb{D}(X)$ has a left adjoint; see [12, Definition 1.13].

(iv) A derivator $\mathbb{D}$ is called triangulated or stable if it is pointed and if every global commutative square is cartesian exactly when it is cocartesian; see [12, Definition 1.15].

A strong derivator is the same thing as a small homotopy theory in the sense of Heller [22]. Thanks to [10, Proposition 2.15], if $\mathcal{M}$ is a Quillen model category its associated derivator $\mathrm{HO}(\mathcal{M})$ is strong. Moreover, if sequential homotopy colimits commute with finite products and homotopy pullbacks in $\mathcal{M}$, the associated derivator $\mathrm{HO}(\mathcal{M})$ is regular. Notice that if $\mathcal{M}$ is pointed, then the derivator $\mathrm{HO}(\mathcal{M})$ is pointed. Finally, a pointed Quillen model category $\mathcal{M}$ is stable if and only if its associated derivator $\mathrm{HO}(\mathcal{M})$ is triangulated.

A.4. Kan extensions. - Given a small category $A$, we denote by $\operatorname{Hot}_{A}=\mathrm{HO}(\mathrm{s} \widehat{A})$ the derivator associated to the projective model category structure on the category of simplicial presehaves. We then have a Yoneda embedding

$$
h: \underline{A} \longrightarrow \operatorname{Hot}_{A} \text {. }
$$

Let $\mathbb{D}$ be a derivator. A 2-functorial version of the Yoneda lemma gives a canonical equivalence of categories

$$
\underline{\operatorname{Hom}}(\underline{A}, \mathbb{D}) \simeq \mathbb{D}\left(A^{\text {op }}\right)
$$


Theorem A.1. - The morphism of prederivators (A.4.1) is the universal morphism from $\underline{A}$ to a derivator. In other words, given any derivator $\mathbb{D}$, the induced functor

$$
h^{*}: \underline{\operatorname{Hom}}_{!}\left(\operatorname{Hot}_{A}, \mathbb{D}\right) \stackrel{\sim}{\longrightarrow} \underline{\operatorname{Hom}}(\underline{A}, \mathbb{D})
$$

is an equivalence of categories.

Proof. — See [9, Corollaire 3.26].

A.5. Monoidal structures. - Thanks to [9, Proposition 5.2] the 2-category of prederivators form a closed symmetric monoidal 2-category with respect to the cartesian product. Given two prederivators $\mathbb{D}$ and $\mathbb{D}^{\prime}$, we denote by $\operatorname{Hom}\left(\mathbb{D}, \mathbb{D}^{\prime}\right)$ the corresponding internal Hom; see [9, §5.1].

Given a prederivator $\mathbb{D}$, by a symmetric monoidal structure on $\mathbb{D}$ we mean a structure of symmetric pseudo monoid on $\mathbb{D}$. In other words, for every small category $X$, $\mathbb{D}(X)$ is a symmetric monoidal category, and for every functor $u: X \rightarrow Y$ between small categories, the inverse image functor

$$
u^{*}: \mathbb{D}(u): \mathbb{D}(Y) \longrightarrow \mathbb{D}(X)
$$

is symmetric monoidal; see $[9, \S 5.4]$. A symmetric monoidal prederivator is a prederivator endowed with a symmetric monoidal structure. Given symmetric monoidal prederivators $\mathbb{D}$ and $\mathbb{D}^{\prime}$, we denote by $\underline{\operatorname{Hom}}^{\otimes}\left(\mathbb{D}, \mathbb{D}^{\prime}\right)$ the category of symmetric monoidal morphisms; see $[9, \S 5.11]$ for details. A symmetric monoidal derivator is a symmetric monoidal prederivator $\mathbb{D}$ which is also a derivator, and such that the tensor product preserves homotopy colimits in each variable, i.e. sucht that, for any object $X \in \mathbb{D}(e)$ the induced morphism

$$
X \otimes-: \mathbb{D} \longrightarrow \mathbb{D}
$$

preserves homotopy colimits.

Given symmetric monoidal derivators $\mathbb{D}$ and $\mathbb{D}^{\prime}$, we denote by $\underline{\text { Hom }}_{!}^{\otimes}\left(\mathbb{D}, \mathbb{D}^{\prime}\right)$ the category of symmetric monoidal morphisms which preserve homotopy colimits.

A basic example of a symmetric monoidal prederivator is given as follows: let $\mathcal{M}$ be a symmetric monoidal category (with monoidal product $-\otimes-$ ) and $\mathcal{W}$ a class of morphisms in $\mathcal{M}$. If the monoidal product preserves the class $\mathcal{W}$, i.e. if we have an inclusion $\mathcal{W} \otimes \mathcal{W} \subseteq \mathcal{W}$, then the prederivator $\underline{\mathcal{M}}\left[\mathcal{W}^{-1}\right]$ of $\S \mathrm{A} .1$ is naturally a symmetric monoidal prederivator. Moreover, if $F:(\mathcal{M}, \mathcal{W}) \rightarrow(\mathcal{N}, \mathcal{V})$ is a symmetric monoidal functor such that $F(\mathcal{W}) \subset \mathcal{V}$, then the induced morphism

$$
\underline{\mathcal{M}}\left[\mathcal{W}^{-1}\right] \longrightarrow \underline{\mathcal{N}}\left[\mathcal{V}^{-1}\right]
$$

is symmetric monoidal.

As for examples of symmetric monoidal derivators, most of them are obtained from symmetric monoidal model categories [24, Definition 4.2.6]. 
Proposition A.2. - Let $\mathcal{M}$ be a symmetric monoidal model category. Then its associated derivator $\mathrm{HO}(\mathcal{M})$ carries a symmetric monoidal structure. Moreover, any symmetric monoidal left Quillen functor between symmetric monoidal model categories induces a symmetric monoidal morphism between the associated derivators.

Proof. - See [9, Proposition 6.1].

A.6. Derived Day convolution product. - Let $A$ be a small symmetric monoidal category. Then $\underline{A}$ is a symmetric monoidal prederivator. Moreover, as s $\widehat{A}$ is then a symmetric monoidal model category (see Theorem 5.2), the derivator $\operatorname{Hot}_{A}$ is then a symmetric monoidal derivator, in such a way that the Yoneda embedding (A.4.1) is a symmetric monoidal morphism of prederivators. Given a symmetric monoidal derivator $\mathbb{D}$, we thus have an induced functor

$$
h^{*}: \underline{\operatorname{Hom}}_{!}^{\otimes}\left(\operatorname{Hot}_{A}, \mathbb{D}\right) \longrightarrow \underline{\operatorname{Hom}}^{\otimes}(\underline{A}, \mathbb{D}) .
$$

Theorem A.3. - The functor (A.6.1) is an equivalence of categories.

Proof. - This is simply a variation on Theorem A.1. Given two derivators $\mathbb{D}^{\prime}$ and $\mathbb{D}^{\prime \prime}$, the derivator $\operatorname{Hom}_{!}\left(\mathbb{D}^{\prime}, \mathbb{D}^{\prime \prime}\right)$ is defined by

$$
\operatorname{Hom}_{!}\left(\mathbb{D}^{\prime}, \mathbb{D}^{\prime \prime}\right)(A)=\underline{\operatorname{Hom}_{!}}\left(\mathbb{D}^{\prime}, \mathbb{D}_{A}^{\prime \prime}\right),
$$

where $\mathbb{D}_{A}^{\prime \prime}$ is in turn the derivator of "presheaves on $A$ with values in $A$ ", i.e. the derivator defined by

$$
\mathbb{D}_{A}^{\prime \prime}(X)=\mathbb{D}^{\prime \prime}(A \times X) .
$$

For a third derivator $\mathbb{D}$, the data of a morphism of prederivators

$$
\mathbb{D} \times \mathbb{D}^{\prime} \longrightarrow \mathbb{D}^{\prime \prime}
$$

which preserves homotopy colimits in each variable is equivalent to the data of an object in $\underline{\operatorname{Hom}}_{!}\left(\mathbb{D}, \mathbf{H o m}_{!}\left(\mathbb{D}^{\prime}, \mathbb{D}^{\prime \prime}\right)\right)$; see $\left[9\right.$, Lemme 5.18]. Moreover, when $\mathbb{D}^{\prime}$ is of the form $\operatorname{Hot}_{B}$, with $B$ a small category, it follows immediately from Theorem A.1 that we have equivalences of derivators

$$
\operatorname{Hom}_{!}\left(\operatorname{Hot}_{B}, \mathbb{D}^{\prime \prime}\right) \simeq \operatorname{Hom}\left(\underline{B}, \mathbb{D}^{\prime \prime}\right) \simeq \mathbb{D}_{B^{\text {op }}}^{\prime} .
$$

Hence, if $A$ is another small category, we obtain canonical equivalences of categories:

$$
\begin{aligned}
\underline{\operatorname{Hom}}_{!}\left(\operatorname{Hot}_{A}, \operatorname{Hom}_{!}\left(\operatorname{Hot}_{B}, \mathbb{D}^{\prime \prime}\right)\right) & \simeq \underline{\operatorname{Hom}}\left(\underline{A}, \operatorname{Hom}_{!}\left(\operatorname{Hot}_{B}, \mathbb{D}^{\prime \prime}\right)\right) \\
& \simeq \underline{\operatorname{Hom}}\left(\underline{A}, \operatorname{Hom}\left(\underline{B}, \mathbb{D}^{\prime \prime}\right)\right) \\
& \simeq \underline{\operatorname{Hom}}\left(\underline{A} \times \underline{B}, \mathbb{D}^{\prime \prime}\right)=\mathbb{D}^{\prime \prime}\left(A^{\mathrm{op}} \times B^{\mathrm{op}}\right) .
\end{aligned}
$$

For $A=B$ and $\mathbb{D}^{\prime \prime}=\operatorname{Hot}_{A}$, we note that the tensor product on $\operatorname{Hot}_{A}$ corresponds, under these equivalences of categories, to the tensor product $\otimes: A \times A \longrightarrow A$ composed with the Yoneda embeding (A.4.1).

More generally, we obtain (by induction on $n \geq 0$ ) that for any $n$-tuple of small categories $\left(A_{1}, \ldots, A_{n}\right)$, the category of morphisms $\operatorname{Hot}_{A_{1}} \times \cdots \times \operatorname{Hot}_{A_{n}} \longrightarrow \mathbb{D}^{\prime \prime}$ 
which preserve homotopy colimits in each variable is canonically equivalent to the category of morphisms $A_{1} \times \cdots \times A_{n} \longrightarrow \mathbb{D}^{\prime \prime}$. This fact implies that the symmetric monoidal structure on $\bar{A}$ extends uniquely to a symmetric monoidal structure on the derivator $\operatorname{Hot}_{A}$. Moreover, the category of symmetric monoidal morphisms from $\operatorname{Hot}_{A}$ to $\mathbb{D}^{\prime \prime}$, which preserve homotopy colimits, is canonically equivalent to the category of symmetric monoidal morphisms from $\underline{A}$ to $\mathbb{D}^{\prime \prime}$.

A.7. Left Bousfield localization. - Let $\mathbb{D}$ be a derivator and $S$ a class of morphisms in the base category $\mathbb{D}(e)$. We say that the derivator $\mathbb{D}$ admits a left Bousfield localization with respect to the class $S$, if there exists a morphism of derivators

$$
\gamma: \mathbb{D} \longrightarrow \mathrm{L}_{S} \mathbb{D}
$$

which commutes with homotopy colimits, sends the elements of $S$ to isomorphisms in $L_{S} \mathbb{D}(e)$, and satisfies the following universal property: given any derivator $\mathbb{D}^{\prime}$, the morphism $\gamma$ induces an equivalence of categories

$$
\gamma^{*}: \underline{\operatorname{Hom}}_{!}\left(\mathrm{L}_{S} \mathbb{D}, \mathbb{D}^{\prime}\right) \stackrel{\sim}{\longrightarrow} \underline{\operatorname{Hom}}_{1, S}\left(\mathbb{D}, \mathbb{D}^{\prime}\right),
$$

where $\underline{\operatorname{Hom}}_{!, S}\left(\mathbb{D}, \mathbb{D}^{\prime}\right)$ denotes the category of morphisms of derivators which commute with homotopy colimits and send the elements of $S$ to isomorphisms in $\mathbb{D}^{\prime}(e)$.

Theorem A.4. - Let $\mathcal{M}$ be a left proper cellular model category and $S$ a set of maps in the homotopy category $\mathrm{Ho}(\mathcal{M})$ of $\mathcal{M}$. Consider the left Bousfield localization $\mathrm{L}_{S} \mathcal{M}$ of $\mathcal{M}$ with respect to the set $S$, i.e. to perform the localization we choose in $\mathcal{M}$ a representative for each element of $S$. Then, the induced morphism of derivators $\mathrm{HO}(\mathcal{M}) \rightarrow \mathrm{HO}\left(\mathrm{L}_{S} \mathcal{M}\right)$ is a left Bousfield localization of the derivator $\mathrm{HO}(\mathcal{M})$ with respect to the set $S$. Moreover, we have a natural adjunction of derivators:



Proof. — See [42, Theorem 4.4].

Remark A.5. - If the domains and codomains of the elements of the set $S$ are homotopically finitely presented (see Definition 3.2), the morphism $\mathrm{HO}\left(\mathrm{L}_{S} \mathcal{M}\right) \rightarrow$ $\mathrm{HO}(\mathcal{M})$ (right adjoint to the localizing functor) preserves filtered homotopy colimits. Therefore, under these hypothesis, if $\mathrm{HO}(\mathcal{M})$ is regular so it is $\mathrm{HO}\left(\mathrm{L}_{S} \mathcal{M}\right)$.

By [42, Lemma 4.3], the Bousfield localization $L_{S} \mathbb{D}$ of a triangulated derivator $\mathbb{D}$ remains triangulated as long as $S$ is stable under the loop space functor. For more general $S$, to remain in the world of triangulated derivators, one has to localize with respect to the set $\Omega(S)$ generated by $S$ and loops, as follows. 
Proposition A.6. - Let $\mathbb{D}$ be a triangulated derivator and $S$ a class of morphisms in $\mathbb{D}(e)$. Let us denote by $\Omega(S)$ the smallest class of morphisms in $\mathbb{D}(e)$ which contains $S$ and is stable under the loop space functor $\Omega: \mathbb{D}(e) \rightarrow \mathbb{D}(e)$. Then for any triangulated derivator $\mathbb{T}$, we have an equality of categories

$$
\underline{\operatorname{Hom}}_{!, \Omega(S)}(\mathbb{D}, \mathbb{T})=\underline{\operatorname{Hom}}_{!, S}(\mathbb{D}, \mathbb{T}) \text {. }
$$

As a consequence, whenever $\mathrm{L}_{\Omega(S)} \mathbb{D}$ exists, this is the triangulated left Bousfied localization of $\mathbb{D}$ with respect to $S$.

Proof. - For $F$ an element of $\underline{\operatorname{Hom}}_{!}(\mathbb{D}, \mathbb{T})$, the functor $F(e): \mathbb{D}(e) \rightarrow \mathbb{T}(e)$ commutes with homotopy colimits, hence it commutes in particular with the suspension functor. Since both $\mathbb{D}$ and $\mathbb{T}$ are triangulated, suspension and loop space functors are inverse to each other. Hence $F(e)$ also commutes with $\Omega$. It is then obvious that $F(e)$ sends $S$ to isomorphisms if and only if it does so with $\Omega(S)$.

Theorem A.7 (Dugger). — Let $\mathcal{M}$ be a combinatorial model category. Then, there exists a small category $A$ and a small set of maps $S$ in $\operatorname{Hot}_{A}(e)=\operatorname{Ho}(\mathrm{s} \widehat{A})$, such that $\mathrm{HO}(\mathcal{M})$ is equivalent to $\mathrm{L}_{S} \operatorname{Hot}_{A}$.

Proof. - This follows from [14, Proposition 3.3] and from Theorem A.4 applied to the projective model structure on the category of simplicial presheaves of a small category.

Remark A.8. - It follows immediately from Theorem A.7 that the statement of Theorem A.4 holds also for left proper combinatoriel model categories. In particular, any derivator which is equivalent to a derivator associated to a combinatorial model category admits a left Bousfield localization with respect to any small set of maps.

Proposition A.9. - Let $\mathbb{D}$ be a symmetric monoidal derivator, and $S$ a class of maps in $\mathbb{D}(e)$. Assume that $S$ is closed under tensor product in $\mathbb{D}$, and that the left Bousfield localization of $\mathbb{D}$ by $S$ exists. Then, $\mathrm{L}_{S} \mathbb{D}$ is symmetric monoidal, and the localization morphism $\gamma: \mathbb{D} \longrightarrow \mathrm{L}_{S} \mathbb{D}$ is symmetric monoidal. Moreover, given any symmetric monoidal derivator $\mathbb{D}^{\prime}$, the induced functor

$$
\gamma^{*}: \underline{\operatorname{Hom}}_{!}^{\otimes}\left(\mathrm{L}_{S} \mathbb{D}, \mathbb{D}^{\prime}\right) \longrightarrow \underline{\operatorname{Hom}}_{!}^{\otimes}\left(\mathbb{D}, \mathbb{D}^{\prime}\right)
$$

is fully-faithful, and its essential image consists of the symmetric monoidal homotopy colimit preserving morphisms which send $S$ to isomorphisms.

Proof. - This is an immediate consequence of the universal property of $L_{S} \mathbb{D}$. The details are left as an exercise for the reader. 
A.8. Stabilization. - Let $\mathbb{D}$ be a derivator. Then there is a universal pointed derivator $\mathbb{D} \rightarrow \mathbb{D}_{\bullet}$ : given any pointed derivator $\mathbb{D}^{\prime}$, the induced functor

$$
\underline{\operatorname{Hom}}_{!}\left(\mathbb{D} \text { •, } \mathbb{D}^{\prime}\right) \stackrel{\sim}{\longrightarrow} \underline{\operatorname{Hom}}_{!}\left(\mathbb{D}, \mathbb{D}^{\prime}\right)
$$

is an equivalence of categories; see [9, Corollaire 4.19]. Using the explicit construction of $\mathbb{D}$ • (see $[9, \S 4.5])$ it is easy to see that when $\mathbb{D}$ • is strong (resp. regular) so is $\mathbb{D}$. If $\mathbb{D}=\mathrm{HO}(\mathcal{M})$ for some model category $\mathcal{M}$, then $\mathbb{D}_{\bullet}$ is equivalent to $\mathrm{HO}\left(\mathcal{M}_{\bullet}\right)$, where $\mathcal{M}$. denotes the model category of pointed objects in $\mathcal{M}$; see [24, Proposition 1.1.8].

Let $\mathbb{D}$ be a pointed derivator. A stabilization of $\mathbb{D}$ is a homotopy colimit preserving morphism stab : $\mathbb{D} \rightarrow \operatorname{St}(\mathbb{D})$, with $\mathrm{St}(\mathbb{D})$ a triangulated strong derivator, which is universal for these properties: given any triangulated strong derivator $\mathbb{T}$, the induced functor

$$
\operatorname{stab}^{*}: \underline{\operatorname{Hom}}_{!}(\operatorname{St}(\mathbb{D}), \mathbb{T}) \stackrel{\sim}{\longrightarrow} \underline{\operatorname{Hom}}_{!}(\mathbb{D}, \mathbb{T}) .
$$

is an equivalence of categories.

Theorem A.10 (Heller [22]). - Any pointed regular strong derivator admits a stabilization.

Given a pointed simplicial model category $\mathcal{M}$, the second named author compared in $[42, \S 8]$ the derivator associated to the model category $\mathrm{Sp}^{\mathbb{N}}(\mathcal{M})$ of $S^{1}$-spectra on $\mathcal{M}$ with the stabilization of the derivator associated to the model category $\mathcal{M}$.

Proposition A.11. - Let $\mathcal{M}$ be a pointed, simplicial, left proper, cellular model category. Assume that sequential homotopy colimits commute with finite products and homotopy pullbacks. Then, the induced morphism of triangulated strong derivators

$$
\mathrm{St}(\mathrm{HO}(\mathcal{M})) \stackrel{\sim}{\longrightarrow} \mathrm{HO}\left(\mathrm{Sp}^{\mathbb{N}}(\mathcal{M})\right)
$$

is an equivalence.

Proof. - See [42, Theorem 8.7].

Let $\mathbb{D}$ be a pointed strong derivator (see $\S A .3$ ) and $S$ be a class of morphisms in $\mathbb{D}(e)$. Assume that $\mathbb{D}$ admits a left Bousfield localization $\mathrm{L}_{S} \mathbb{D}$ with respect to $S$; see $\S A .7$. Assume also that the stablization $\mathrm{St}(\mathbb{D})$ of $\mathbb{D}$ exists.

We then have two homotopy colimit preserving morphisms:

$$
\mathrm{L}_{S} \mathbb{D} \stackrel{\gamma}{\longleftarrow} \mathbb{D} \stackrel{\text { stab }}{\longrightarrow} \mathrm{St}(\mathbb{D}) .
$$

By examining the relevant universal properties, we obtain the following result.

Proposition A.12. - Under the above assumptions, the derivator $\mathrm{L}_{\Omega(\operatorname{stab}(S))} \mathrm{St}(\mathbb{D})$ exists if and only if the derivator $\operatorname{St}\left(\mathrm{L}_{S} \mathbb{D}\right)$ exists. Moreover, if this is the case, then $\mathrm{L}_{\Omega(\operatorname{stab}(S))} \mathrm{St}(\mathbb{D}) \simeq \mathrm{St}\left(\mathrm{L}_{S} \mathbb{D}\right)$ under $\mathbb{D}$. 
Corollary A.13. - Let $\mathcal{M}$ be a pointed left proper combinatorial model category. Then the stabilization $\mathrm{St}(\mathrm{HO}(\mathcal{M}))$ of $\mathrm{HO}(\mathcal{M})$ exists and is equivalent to the derivator associated to a stable combinatorial model category.

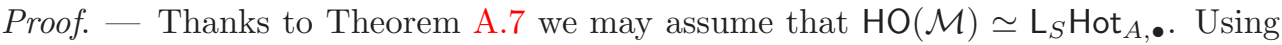
Proposition A.12 and Theorem A.4, we see it is sufficient to treat the case where $S$ is the empty set. Proposition A.11 allow us then to conclude the proof.

Remark A.14. - A careful analysis of the proof of the Corollary A.13 will lead to a proof of Proposition A.11 for any simplicial combinatorial model category $\mathcal{M}$. Note that since any combinatorial model category is equivalent to a simplicial one, we conclude the existence of stabilizations for any derivator associated to a combinatorial model category; however, we will not need this level of generality.

Theorem A.15. - Let $A$ be a small symmetric monoidal category. Then there is a unique symmetric monoidal structure on the triangulated derivator $\mathrm{St}\left(\operatorname{Hot}_{A, \bullet}\right)$ whose tensor product preserves homotopy colimits in each variables, such that the composed morphism

$$
\underline{A} \longrightarrow \operatorname{Hot}_{A} \longrightarrow \mathrm{St}\left(\operatorname{Hot}_{A, \bullet}\right)
$$

is symmetric monoidal. Moreover, given any strong triangulated derivator $\mathbb{D}$, the induced functor

$$
\underline{\operatorname{Hom}}_{!}^{\otimes}\left(\operatorname{St}\left(\operatorname{Hot}_{A, \bullet}\right), \mathbb{D}\right) \stackrel{\sim}{\longrightarrow} \underline{\operatorname{Hom}}^{\otimes}(\underline{A}, \mathbb{D})
$$

is an equivalence of categories.

Proof. - It follows immediately from Theorem A.1 and from the universal property of $\operatorname{St}\left(\operatorname{Hot}_{A, \bullet}\right)$ that, for any small category $A$ and any strong triangulated derivator $\mathbb{D}$, we have canonical equivalences of categories:

$$
\underline{\operatorname{Hom}}_{!}\left(\operatorname{St}\left(\operatorname{Hot}_{A, \bullet}\right), \mathbb{D}\right) \simeq \underline{\operatorname{Hom}}(\underline{A}, \mathbb{D}) \simeq \mathbb{D}\left(A^{\text {op }}\right) .
$$

Starting from this point on, the proof of Theorem A.3 holds here mutatis mutandis.

Remark A.16. - The derivator $\mathrm{St}\left(\operatorname{Hot}_{A, \bullet}\right)$ is equivalent to the derivator associated to the model category of symmetric spectra in the category of pointed simplicial presheaves on $A$. This equivalence defines a symmetric monoidal structure on $\operatorname{St}\left(\operatorname{Hot}_{A, \bullet}\right)$, making the morphism

$$
\underline{A} \longrightarrow \operatorname{Hot}_{A} \longrightarrow \mathrm{St}\left(\operatorname{Hot}_{A, \bullet}\right)
$$

symmetric monoidal; see Proposition 5.3, Theorem 6.1, and Proposition A.11. This monoidal structure coincides with the one of Theorem A.15, thanks to the uniqueness of the latter. 
A.9. Spectral enrichment. - Recall from [11, Appendix A.3] that any triangulated derivator $\mathbb{D}$ is canonically enriched over spectra, i.e. we have a morphism of derivators

$$
\mathbb{R H o m}(-,-): \mathbb{D}^{\mathrm{op}} \times \mathbb{D} \longrightarrow \mathrm{HO}\left(\mathrm{Sp}^{\mathbb{N}}\right) .
$$

Moreover, this enrichment over spectra is compatible with adjunctions: given an adjunction

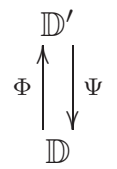

we have a canonical isomorphism in the stable homotopy category of spectra:

$$
\mathbb{R H o m}_{\mathbb{D}^{\prime}}(\Phi X, Y) \simeq \mathbb{R} \operatorname{Hom}_{\mathbb{D}}(X, \Psi Y) \quad X \in \mathbb{D}, Y \in \mathbb{D}^{\prime} .
$$

\section{References}

[1] P. Berthelot, A. Grothendieck, and L. Illusie, Théorie des intersections et théorème de Riemann-Roch (SGA 6), Lectures Notes in Mathematics 225, Springer-Verlag, 1971.

[2] A. Blumberg and M. Mandell, Localization theorems in topological Hochschild homology and topological cyclic homology. Available at arXiv:0802.3938.

[3] A. Bondal and M. Kapranov, Framed triangulated categories (Russian) Mat. Sb. 181 (1990) no. 5, 669-683; translation in Math. USSR-Sb. 70 no. 1, 93-107.

[4] A. Bondal and M. Van den Bergh, Generators and Representability of Functors in commutative and Noncommutative Geometry. Moscow Mathematical Journal 3(1), 1-37 (2003)

[5] F. Borceux, Handbook of categorical algebra 2. Encyclopedia of Mathematics and its Applications, 51, Cambridge University Press, 1994.

[6] A. K. Bousfield and D. Kan, Homotopy limits, completions, and localizations. Lecture Notes in Mathematics 304, Springer-Verlag, 1972.

[7] D.-C. Cisinski, Images directes cohomologiques dans les catégories de modèles. Annales Mathématiques Blaise Pascal 10 (2003), 195-244.

[8] _ Les préfaisceaux comme modèles des type d'homotopie. Astérisque 308, Soc. Math. France, 2006.

[9] - Propriétés universelles et extensions de Kan dérivées. Theory and Applications of Categories 20 (2008), no. 17, 605-649.

[10] _ Catégories dérivables. Bull. Soc. Math. France. To appear. Preprint version available at http://www.math.univ-paris13.fr/ cisinski/

[11] D.-C. Cisinski and G. Tabuada, Non-connective $K$-theory via universal invariants. Available at arXiv:0903.3717v2.

[12] D.-C. Cisinski and A. Neeman, Additivity for derivator K-theory. Adv. Math. 217 (2008), no. 4, 1381-1475.

[13] B. Day, On closed categories of functors. Reports of the midwest category seminar IV. Lecture notes in Math. 137 (1970), 1-38.

[14] D. Dugger, Combinatorial model categories have presentations. Adv. Math. 164 (2001), no. $1,177-201$.

[15] Universal homotopy theories. Adv. Math. 164 (2001), no. 1, 144-176. 
[16] V. Drinfeld, DG quotients of DG categories. J. Algebra 272 (2004), 643-691.

[17] — DG categories. University of Chicago Geometric Langlands Seminar. Available at http://www.math.utexas. edu/users/benzvi/GRASP/lectures/Langlands.html.

[18] W. G. Dwyer and D. M. Kan, Equivalences between homotopy theories of diagrams, Algebraic topology and algebraic $K$-theory, Annals of Math. Studies, vol. 113, Princeton University Press, 1987, pp. 180-205.

[19] S. Eilenberg and J. C. Moore, Homology and fibrations I. Coalgebras, cotensor product and its derived functors, Comment. Math. Helv. 40 (1966), 199-236.

[20] P. Goerss and J. Jardine, Simplicial homotopy theory. Progress in Mathematics, 174, 1999.

[21] A. Heller, Homotopy theories. Mem. Amer. Math. Soc. 71 (1988), no. 383.

[22] Stable homotopy theories and stabilization. J. Pure Appl. Algebra 115 (1997), $113-130$.

[23] P. Hirschhorn, Model categories and their localizations. Mathematical Surveys and Monographs, 99, American Mathematical Society, 2003.

[24] M. Hovey, Model categories. Mathematical Surveys and Monographs, 63, American Mathematical Society, 1999.

[25] _ Spectra and symmetric spectra in general model categories. J. Pure Appl. Algebra 165, 2001, 63-127.

[26] _ Model category structures on chain complexes of sheaves. Trans. Amer. Math. Soc. 353 (2001), no.6, 2441-2457.

[27] M. Hovey, B. Shipley and J. Smith, Symmetric spectra. J. Amer. Math. Soc. 13 (2000), no. $1,149-208$.

[28] A. Grothendieck, Les Dérivateurs. Available at http://people.math.jussieu.fr/maltsin/groth/Derivateurs.html.

[29] C. Kassel, Cyclic homology, comodules and mixed complexes, J. Algebra 107, (1987), $195-216$

[30] B. Keller, On differential graded categories. International Congress of Mathematicians (Madrid), Vol. II, 151-190, Eur. Math. Soc., Zürich, 2006.

[31] - On the cyclic homology of exact categories. J. Pure Appl. Algebra 136 (1999), no. $1,1-56$.

[32] On the cyclic homology of ringed spaces and schemes. Doc. Math. 3 (1998), 231-259 (electronic).

[33] M. Kontsevich, Non-commutative motives. Talk at the Institute for Advanced Study on the occasion of the 61st birthday of Pierre Deligne, October 2005. Video available at http://video.ias.edu/Geometry-and-Arithmetic.

[34] _ Notes on motives in finite characteristic. Available at arXiv:0702206. To appear in "Manin's Festschrift". Editors Yu. Zarkhin and Yu. Tschinkel.

[35] — Categorification, NC Motives, Geometric Langlands and Lattice Models. Talk at the University of Chicago Geometric Langlands Seminar (2006). Available at http://www . math.utexas . edu/users/benzvi/notes.html.

[36] M. Kontsevich and Y. Soibelmann, Notes on A-infinity algebras, A-infinity categories and non-commutative geometry I. In "Homological Mirror Symmetry: New Developments and Perspectives" (A.Kapustin et al. (Eds.)). Lect. Notes in Physics, 757, Springer, Berlin Heidelberg, 2009, 153-219.

[37] J. P. May, The additivity of traces in triangulated categories, Adv. Math. 163 (2001), no. $1,34-73$. 
[38] J.-L. Loday, Cyclic homology. Volume 301 of Grundlehren der Mathematischen Wissenschaften. Springer-Verlag, Berlin, 1992.

[39] A. Neeman, Triangulated categories. Annals of Mathematics Studies, 148, Princeton University Press, 2001.

[40] D. Quillen, Homotopical algebra. Lecture Notes in Mathematics 43, Springer-Verlag, 1967.

[41] M. Schlichting, Negative K-theory of derived categories. Math. Z. 253 (2006), no. 1, 97-134.

[42] G. Tabuada, Higher K-theory via universal invariants. Duke Math. J. 145 (2008), no.1, $121-206$.

[43] _ Invariants additifs de dg-catégories. Int. Math. Res. Not. 53 (2005), 3309-3339.

[44] _ Une structure de catégorie de modèles de Quillen sur la catégorie des dgcatégories, C. R. Math. Acad. Sci. Paris 340 (2005), no. 1, 15-19.

[45] - Generalized spectral categories, topological Hochschild homology, and trace maps. Algebraic and Geometric Topology, 10 (2010), 137-213.

[46] - Matrix invariants of Spectral categories. Int. Math. Res. Not., doi:10.1093/imrn/rnp219. In press.

[47] R.W. Thomason and T. Trobaugh, Higher algebraic K -theory of schemes and of derived categories. In The Grothendieck Festschrift, Volume III. Volume $\mathbf{8 8}$ of Progress in Math., 247-436. Birkhauser, Boston, Bassel, Berlin, 1990.

[48] B. Toën, The homotopy theory of dg-categories and derived Morita theory. Invent. Math. 167 (2007), no. 3, 615-667.

[49] Lectures on dg-categories. Sedano winter school on K-theory, January 2007.

Available at http://www.math.univ-toulouse.fr/toen/note.html.

[50] Secondary K-theory I-III. Lecture notes from the workshop and conference on Topological Field Theories, Northwestern University, May 2009. Available at http://www . math.utexas. edu/users/benzvi/GRASP/lectures/NWTFT.html.

[51] B. Toën and M. Vaquié, Moduli of objects in dg-categories. Ann. Sci. de l'ENS 40 (2007), Issue $3,387-444$.

[52] B. Toën and G. Vezzosi, A note on Chern character, loop spaces and derived algebraic geometry. Available at arXiv:0804.1274. To appear in the Abel Symposium, Oslo 2007.

[53] J. L. Verdier, Des catégories dérivées des catégories abéliennes, Astérisque 239, Soc. Math. France, 1996.

[54] _ Cyclic homology for schemes. Proc. Amer. Math. Soc. 124 (1996), no. 6, 16551662.

February 3, 2010

D.-C. Cisinski, Institut Galilée, Université Paris 13, 99, Av. Jean-Baptiste Clément, 93430 Villetaneuse, France • E-mail : cisinski@math.univ-paris13.fr Url : http://www-math.univ-paris13.fr/ cisinski/

G. TABuAda, Departamento de Matemática e CMA, FCT-UNL, Quinta da Torre, 2829-516 Caparica, Portugal • E-mail : tabuada@fct.unl.pt 